\title{
Topological Quantum Information Theory
}

\author{
Louis H. Kauffman \\ Department of Mathematics, Statistics \\ and Computer Science (m/c 249) \\ 851 South Morgan Street \\ University of Illinois at Chicago \\ Chicago, Illinois 60607-7045 \\ $<$ kauffman@uic.edu> \\ and \\ Samuel J. Lomonaco Jr. \\ Department of Computer Science and Electrical Engineering \\ University of Maryland Baltimore County \\ 1000 Hilltop Circle, Baltimore, MD 21250 \\ $<$ lomonaco@umbc.edu>
}

\begin{abstract}
This paper is an introduction to relationships between quantum topology and quantum computing. In this paper we discuss unitary solutions to the YangBaxter equation that are universal quantum gates, quantum entanglement and topological entanglement, and we give an exposition of knot-theoretic recoupling theory, its relationship with topological quantum field theory and apply these methods to produce unitary representations of the braid groups that are dense in the unitary groups. Our methods are rooted in the bracket state sum model for the Jones polynomial. We give our results for a large class of representations based on values for the bracket polynomial that are roots of unity. We make a separate and self-contained study of the quantum universal Fibonacci model in this framework. We apply our results to give quantum algorithms for the computation of the colored Jones polynomials for knots and links, and the Witten-Reshetikhin-Turaev invariant of three manifolds.
\end{abstract}




\section{Introduction}

This paper describes relationships between quantum topology and quantum computing. It is a modified version of Chapter 14 of our book [18] and an expanded version of [58]. Quantum topology is, roughly speaking, that part of low-dimensional topology that interacts with statistical and quantum physics. Many invariants of knots, links and three dimensional manifolds have been born of this interaction, and the form of the invariants is closely related to the form of the computation of amplitudes in quantum mechanics. Consequently, it is fruitful to move back and forth between quantum topological methods and the techniques of quantum information theory.

We sketch the background topology, discuss analogies (such as topological entanglement and quantum entanglement), show direct correspondences between certain topological operators (solutions to the Yang-Baxter equation) and universal quantum gates. We then describe the background for topological quantum computing in terms of Temperley-Lieb (we will sometimes abbreviate this to $T L$ ) recoupling theory. This is a recoupling theory that generalizes standard angular momentum recoupling theory, generalizes the Penrose theory of spin networks and is inherently topological. Temperley-Lieb recoupling Theory is based on the bracket polynomial model [37, 44] for the Jones polynomial. It is built in terms of diagrammatic combinatorial topology. The same structure can be explained in terms of the $S U(2)_{q}$ quantum group, and has relationships with functional integration and Witten's approach to topological quantum field theory. Nevertheless, the approach given here will be unrelentingly elementary. Elementary, does not necessarily mean simple. In this case an architecture is built from simple beginnings and this archictecture and its recoupling language can be applied to many things including, e.g. colored Jones polynomials, Witten-Reshetikhin-Turaev invariants of three manifolds, topological quantum field theory and quantum computing.

In quantum computing, the application of topology is most interesting because the simplest non-trivial example of the Temperley-Lieb recoupling Theory gives the so-called Fibonacci model. The recoupling theory yields representations of the Artin braid group into unitary groups $U(n)$ where $n$ is a Fibonacci number. These representations are dense in the unitary group, and can be used to model quantum computation universally in terms of representations of the braid group. Hence the term: topological quantum computation.

In this paper, we outline the basics of the Temperely-Lieb Recoupling Theory, and show explicitly how the Fibonacci model arises from it. The diagrammatic computations in the section 11 and 12 are completely self-contained 
and can be used by a reader who has just learned the bracket polynomial, and wants to see how these dense unitary braid group representations arise from it. The outline of the parts of this paper is given below.

1. Knots and Braids

2. Quantum Mechanics and Quantum Computation

3. Braiding Operators and Univervsal Quantum Gates

4. A Remark about EPR, Entanglement and Bell's Inequality

5. The Aravind Hypothesis

6. $S U(2)$ Representations of the Artin Braid Group

7. The Bracket Polynomial and the Jones Polynomial

8. Quantum Topology, Cobordism Categories, Temperley-Lieb Algebra and Topological Quantum Field Theory

9. Braiding and Topological Quantum Field Theory

10. Spin Networks and Temperley-Lieb Recoupling Theory

11. Fibonacci Particles

12. The Fibonacci Recoupling Model

13. Quantum Computation of Colored Jones Polynomials and the WittenReshetikhin-Turaev Invariant

We should point out that while this paper attempts to be self-contained, and hence has some expository material, most of the results are either new, or are new points of view on known results. The material on $S U(2)$ representations of the Artin braid group is new, and the relationship of this material to the recoupling theory is new. The treatment of elementary cobordism categories is well-known, but new in the context of quantum information theory. The reformulation of Temperley-Lieb recoupling theory for the purpose of producing unitary braid group representations is new for quantum information theory, and directly related to much of the recent work of Freedman and his collaborators. The treatment of the Fibonacci model in terms of two-strand recoupling theory is new and at the same time, the most elementary non-trivial example of the recoupling theory. The models in section 10 for quantum computation of colored Jones polynomials and for quantum computation of the Witten-Reshetikhin-Turaev invariant are new in this form of the recoupling theory. They take a particularly simple aspect in this context. 
Here is a very condensed presentation of how unitary representations of the braid group are constructed via topological quantum field theoretic methods. One has a mathematical particle with label $P$ that can interact with itself to produce either itself labeled $P$ or itself with the null label $*$. We shall denote the interaction of two particles $P$ and $Q$ by the expression $P Q$, but it is understood that the "value" of $P Q$ is the result of the interaction, and this may partake of a number of possibilities. Thus for our particle $P$, we have that $P P$ may be equal to $P$ or to $*$ in a given situation. When $*$ interacts with $P$ the result is always $P$. When $*$ interacts with $*$ the result is always $*$. One considers process spaces where a row of particles labeled $P$ can successively interact, subject to the restriction that the end result is $P$. For example the space $V[(a b) c]$ denotes the space of interactions of three particles labeled $P$. The particles are placed in the positions $a, b, c$. Thus we begin with $(P P) P$. In a typical sequence of interactions, the first two $P$ 's interact to produce a $*$, and the $*$ interacts with $P$ to produce $P$.

$$
(P P) P \longrightarrow(*) P \longrightarrow P \text {. }
$$

In another possibility, the first two $P$ 's interact to produce a $P$, and the $P$ interacts with $P$ to produce $P$.

$$
(P P) P \longrightarrow(P) P \longrightarrow P .
$$

It follows from this analysis that the space of linear combinations of processes $V[(a b) c]$ is two dimensional. The two processes we have just described can be taken to be the qubit basis for this space. One obtains a representation of the three strand Artin braid group on $V[(a b) c]$ by assigning appropriate phase changes to each of the generating processes. One can think of these phases as corresponding to the interchange of the particles labeled $a$ and $b$ in the association $(a b) c$. The other operator for this representation corresponds to the interchange of $b$ and $c$. This interchange is accomplished by a unitary change of basis mapping

$$
F: V[(a b) c] \longrightarrow V[a(b c)] .
$$

If

$$
A: V[(a b) c] \longrightarrow V[(b a) c]
$$

is the first braiding operator (corresponding to an interchange of the first two particles in the association) then the second operator

$$
B: V[(a b) c] \longrightarrow V[(a c) b]
$$

is accomplished via the formula $B=F^{-1} R F$ where the $R$ in this formula acts in the second vector space $V[a(b c)]$ to apply the phases for the interchange of 
$b$ and $c$. These issues are illustrated in Figure 1, where the parenthesization of the particles is indicated by circles and by also by trees. The trees can be taken to indicate patterns of particle interaction, where two particles interact at the branch of a binary tree to produce the particle product at the root. See also Figure 28 for an illustration of the braiding $B=F^{-1} R F$

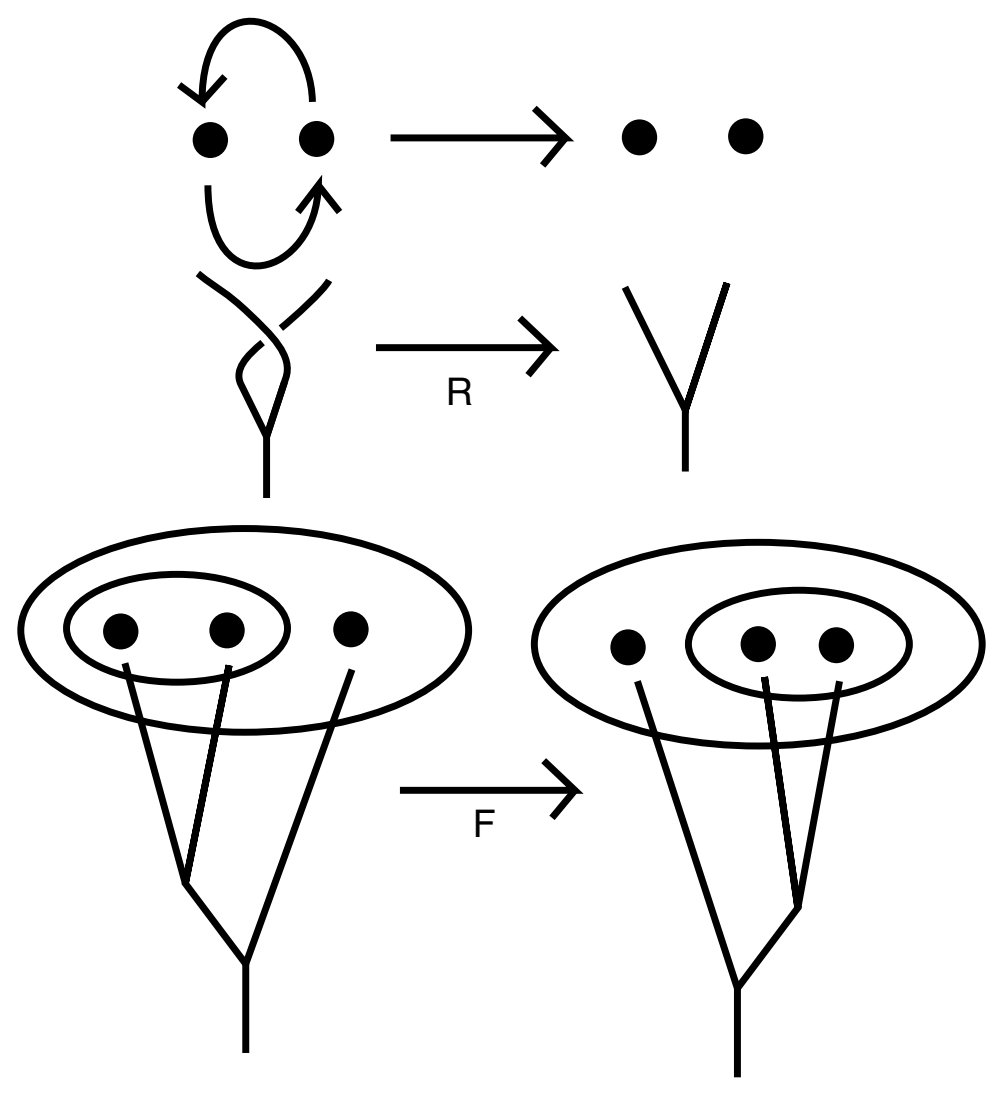

Figure 1 - Braiding Anyons.

In this scheme, vector spaces corresponding to associated strings of particle interactions are interrelated by recoupling transformations that generalize the mapping $F$ indicated above. A full representation of the Artin braid group on each space is defined in terms of the local interchange phase gates and the recoupling transformations. These gates and transformations have to satisfy a number of identities in order to produce a well-defined representation of the braid group. These identities were discovered originally in relation to topological quantum field theory. In our approach the structure of phase gates and recoupling transformations arise naturally from the structure of the bracket 
model for the Jones polynomial. Thus we obtain a knot-theoretic basis for topological quantum computing.

In modeling the quantum Hall effect $[86,26,15,16]$, the braiding of quasiparticles (collective excitations) leads to non-trival representations of the Artin braid group. Such particles are called Anyons. The braiding in these models is related to topological quantum field theory. It is hoped that the mathematics we explain here will form a bridge between theoretical models of anyons and their applications to quantum computing.

Acknowledgement. The first author thanks the National Science Foundation for support of this research under NSF Grant DMS-0245588. Much of this effort was sponsored by the Defense Advanced Research Projects Agency (DARPA) and Air Force Research Laboratory, Air Force Materiel Command, USAF, under agreement F30602-01-2-05022. The U.S. Government is authorized to reproduce and distribute reprints for Government purposes notwithstanding any copyright annotations thereon. The views and conclusions contained herein are those of the authors and should not be interpreted as necessarily representing the official policies or endorsements, either expressed or implied, of the Defense Advanced Research Projects Agency, the Air Force Research Laboratory, or the U.S. Government. (Copyright 2006.) It gives the authors pleasure to thank the Newton Institute in Cambridge England and ISI in Torino, Italy for their hospitality during the inception of this research and to thank Hilary Carteret for useful conversations.

\section{Knots and Braids}

The purpose of this section is to give a quick introduction to the diagrammatic theory of knots, links and braids. A knot is an embedding of a circle in threedimensional space, taken up to ambient isotopy. The problem of deciding whether two knots are isotopic is an example of a placement problem, a problem of studying the topological forms that can be made by placing one space inside another. In the case of knot theory we consider the placements of a circle inside three dimensional space. There are many applications of the theory of knots. Topology is a background for the physical structure of real knots made from rope of cable. As a result, the field of practical knot tying is a field of applied topology that existed well before the mathematical discipline of topology arose. Then again long molecules such as rubber molecules and DNA molecules can be knotted and linked. There have been a number of intense applications of knot theory to the study of $D N A$ [81] and to polymer physics [61]. Knot theory 
is closely related to theoretical physics as well with applications in quantum gravity $[85,78,53]$ and many applications of ideas in physics to the topological structure of knots themselves [44].

Quantum topology is the study and invention of topological invariants via the use of analogies and techniques from mathematical physics. Many invariants such as the Jones polynomial are constructed via partition functions and generalized quantum amplitudes. As a result, one expects to see relationships between knot theory and physics. In this paper we will study how knot theory can be used to produce unitary representations of the braid group. Such representations can play a fundamental role in quantum computing.

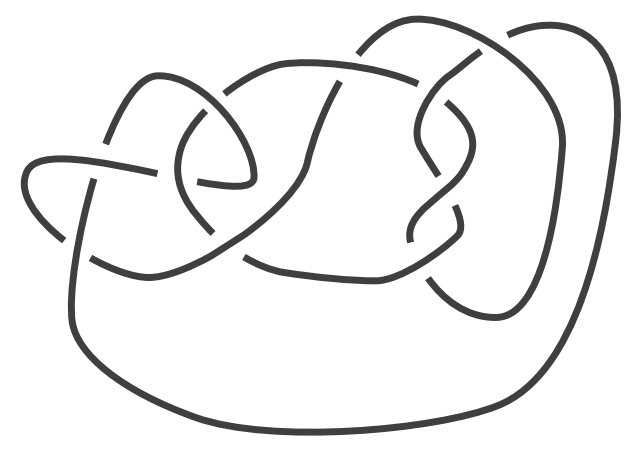

Figure 2 - A knot diagram.

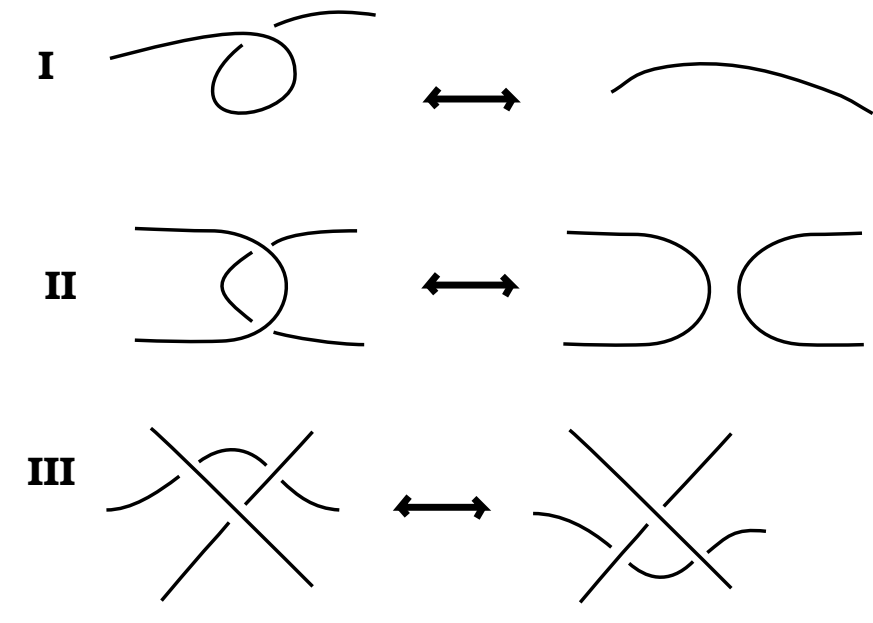

Figure 3 - The Reidemeister Moves. 
That is, two knots are regarded as equivalent if one embedding can be obtained from the other through a continuous family of embeddings of circles in threespace. A link is an embedding of a disjoint collection of circles, taken up to ambient isotopy. Figure 2 illustrates a diagram for a knot. The diagram is regarded both as a schematic picture of the knot, and as a plane graph with extra structure at the nodes (indicating how the curve of the knot passes over or under itself by standard pictorial conventions).

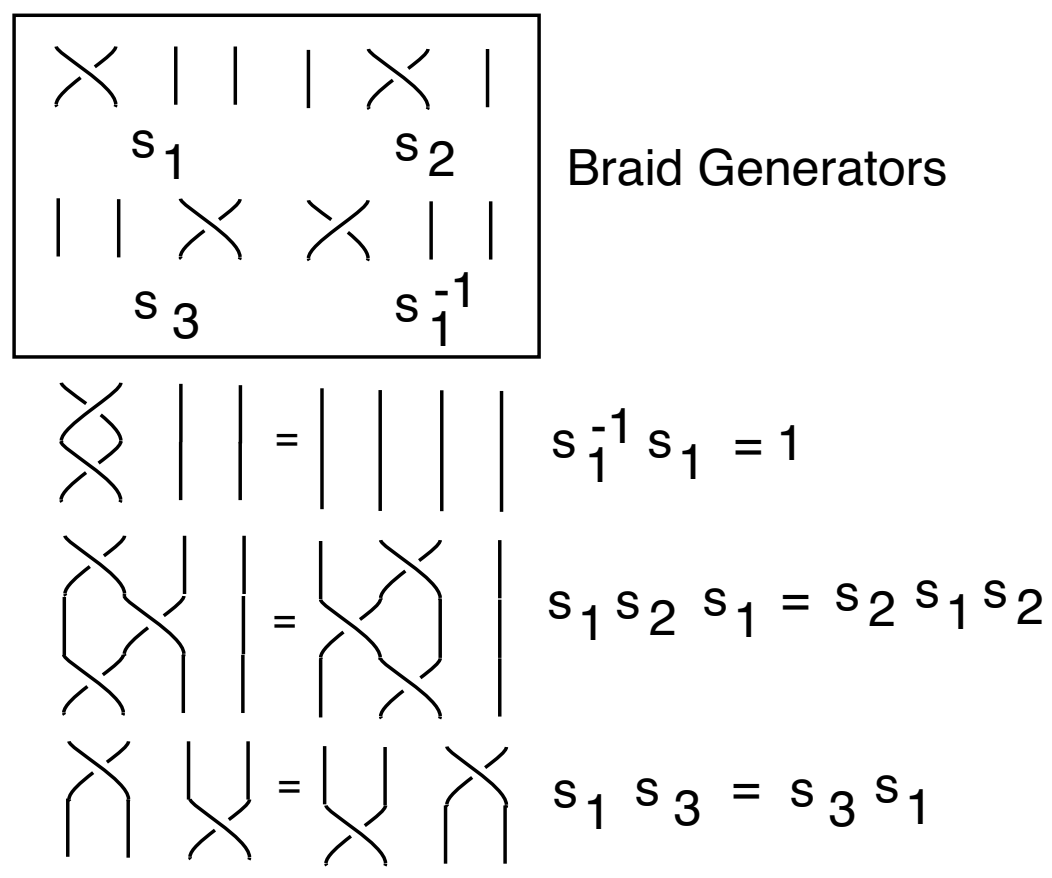

Figure 4 - Braid Generators.

Ambient isotopy is mathematically the same as the equivalence relation generated on diagrams by the Reidemeister moves. These moves are illustrated in Figure 3. Each move is performed on a local part of the diagram that is topologically identical to the part of the diagram illustrated in this figure (these figures are representative examples of the types of Reidemeister moves) without changing the rest of the diagram. The Reidemeister moves are useful in doing combinatorial topology with knots and links, notably in working out the behaviour of knot invariants. A knot invariant is a function defined from knots and links to some other mathematical object (such as groups or polynomials or numbers) such that equivalent diagrams are mapped 
to equivalent objects (isomorphic groups, identical polynomials, identical numbers). The Reidemeister moves are of great use for analyzing the structure of knot invariants and they are closely related to the Artin braid group, which we discuss below.

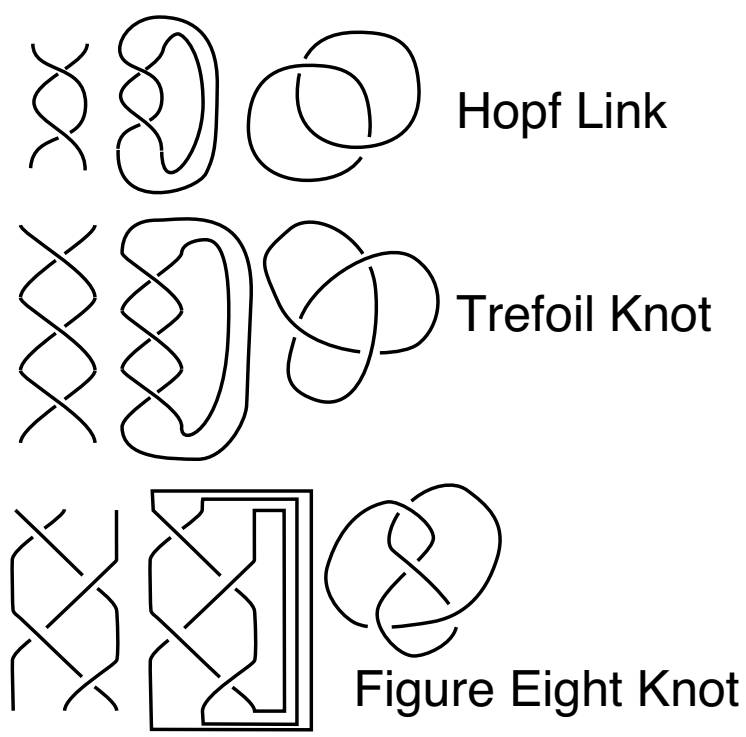

Figure 5 - Closing Braids to form knots and links.

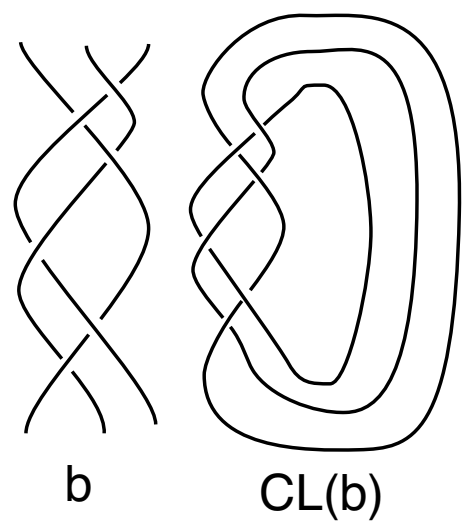

Figure 6 - Borromean Rings as a Braid Closure.

A braid is an embedding of a collection of strands that have their ends in two rows of points that are set one above the other with respect to a choice of 
vertical. The strands are not individually knotted and they are disjoint from one another. See Figures 4, 5 and 6 for illustrations of braids and moves on braids. Braids can be multiplied by attaching the bottom row of one braid to the top row of the other braid. Taken up to ambient isotopy, fixing the endpoints, the braids form a group under this notion of multiplication. In Figure 4 we illustrate the form of the basic generators of the braid group, and the form of the relations among these generators. Figure 5 illustrates how to close a braid by attaching the top strands to the bottom strands by a collection of parallel arcs. A key theorem of Alexander states that every knot or link can be represented as a closed braid. Thus the theory of braids is critical to the theory of knots and links. Figure 6 illustrates the famous Borromean Rings (a link of three unknotted loops such that any two of the loops are unlinked) as the closure of a braid.

Let $B_{n}$ denote the Artin braid group on $n$ strands. We recall here that $B_{n}$ is generated by elementary braids $\left\{s_{1}, \cdots, s_{n-1}\right\}$ with relations

$$
\begin{aligned}
& \text { 1. } s_{i} s_{j}=s_{j} s_{i} \text { for }|i-j|>1, \\
& \text { 2. } s_{i} s_{i+1} s_{i}=s_{i+1} s_{i} s_{i+1} \text { for } i=1, \cdots n-2 .
\end{aligned}
$$

See Figure 4 for an illustration of the elementary braids and their relations. Note that the braid group has a diagrammatic topological interpretation, where a braid is an intertwining of strands that lead from one set of $n$ points to another set of $n$ points. The braid generators $s_{i}$ are represented by diagrams where the $i$-th and $(i+1)$-th strands wind around one another by a single half-twist (the sense of this turn is shown in Figure 4) and all other strands drop straight to the bottom. Braids are diagrammed vertically as in Figure 4, and the products are taken in order from top to bottom. The product of two braid diagrams is accomplished by adjoining the top strands of one braid to the bottom strands of the other braid.

In Figure 4 we have restricted the illustration to the four-stranded braid group $B_{4}$. In that figure the three braid generators of $B_{4}$ are shown, and then the inverse of the first generator is drawn. Following this, one sees the identities $s_{1} s_{1}^{-1}=1$ (where the identity element in $B_{4}$ consists in four vertical strands), $s_{1} s_{2} s_{1}=s_{2} s_{1} s_{2}$, and finally $s_{1} s_{3}=s_{3} s_{1}$.

Braids are a key structure in mathematics. It is not just that they are a collection of groups with a vivid topological interpretation. From the algebraic point of view the braid groups $B_{n}$ are important extensions of the symmetric groups $S_{n}$. Recall that the symmetric group $S_{n}$ of all permutations of $n$ distinct objects has presentation as shown below. 
1. $s_{i}^{2}=1$ for $i=1, \cdots n-1$,

2. $s_{i} s_{j}=s_{j} s_{i}$ for $|i-j|>1$,

3. $s_{i} s_{i+1} s_{i}=s_{i+1} s_{i} s_{i+1}$ for $i=1, \cdots n-2$.

Thus $S_{n}$ is obtained from $B_{n}$ by setting the square of each braiding generator equal to one. We have an exact sequence of groups

$$
1 \longrightarrow B_{n} \longrightarrow S_{n} \longrightarrow 1
$$

exhibiting the Artin braid group as an extension of the symmetric group.

In the next sections we shall show how representations of the Artin braid group are rich enough to provide a dense set of transformations in the unitary groups. Thus the braid groups are in principle fundamental to quantum computation and quantum information theory.

\section{Quantum Mechanics and Quantum Compu- tation}

We shall quickly indicate the basic principles of quantum mechanics. The quantum information context encapsulates a concise model of quantum theory:

The initial state of a quantum process is a vector $|v\rangle$ in a complex vector space $H$. Measurement returns basis elements $\beta$ of $H$ with probability

$$
|\langle\beta \mid v\rangle|^{2} /\langle v \mid v\rangle
$$

where $\langle v \mid w\rangle=v^{\dagger} w$ with $v^{\dagger}$ the conjugate transpose of $v$. A physical process occurs in steps $|v\rangle \longrightarrow U|v\rangle=|U v\rangle$ where $U$ is a unitary linear transformation.

Note that since $\langle U v \mid U w\rangle=\left\langle v\left|U^{\dagger} U\right| w\right\rangle=\langle v \mid w\rangle=$ when $U$ is unitary, it follows that probability is preserved in the course of a quantum process.

One of the details required for any specific quantum problem is the nature of the unitary evolution. This is specified by knowing appropriate information about the classical physics that supports the phenomena. This information is used to choose an appropriate Hamiltonian through which the unitary operator is constructed via a correspondence principle that replaces classical variables with appropriate quantum operators. (In the path integral approach one needs a Langrangian to construct the action on which the path integral is based.) One needs to know certain aspects of classical physics to solve any specific quantum problem. 
A key concept in the quantum information viewpoint is the notion of the superposition of states. If a quantum system has two distinct states $|v\rangle$ and $|w\rangle$, then it has infinitely many states of the form $a|v\rangle+b|w\rangle$ where $a$ and $b$ are complex numbers taken up to a common multiple. States are "really" in the projective space associated with $H$. There is only one superposition of a single state $|v\rangle$ with itself. On the other hand, it is most convenient to regard the states $|v\rangle$ and $|w\rangle$ as vectors in a vector space. We than take it as part of the procedure of dealing with states to normalize them to unit length. Once again, the superposition of a state with itself is again itself.

Dirac [23] introduced the "bra -(c)-ket" notation $\langle A \mid B\rangle=A^{\dagger} B$ for the inner product of complex vectors $A, B \in H$. He also separated the parts of the bracket into the $b r a<A \mid$ and the ket $|B\rangle$. Thus

$$
\langle A \mid B\rangle=\langle A|| B\rangle
$$

In this interpretation, the ket $|B\rangle$ is identified with the vector $B \in H$, while the bra $<A \mid$ is regarded as the element dual to $A$ in the dual space $H^{*}$. The dual element to $A$ corresponds to the conjugate transpose $A^{\dagger}$ of the vector $A$, and the inner product is expressed in conventional language by the matrix product $A^{\dagger} B$ (which is a scalar since $B$ is a column vector). Having separated the bra and the ket, Dirac can write the "ket-bra" $|A\rangle\langle B|=A B^{\dagger}$. In conventional notation, the ket-bra is a matrix, not a scalar, and we have the following formula for the square of $P=|A\rangle\langle B|$ :

$$
P^{2}=|A\rangle\langle B|| A\rangle\langle B|=A\left(B^{\dagger} A\right) B^{\dagger}=\left(B^{\dagger} A\right) A B^{\dagger}=\langle B \mid A\rangle P .
$$

The standard example is a ket-bra $P=|A\rangle\langle A|$ where $\langle A \mid A\rangle=1$ so that $P^{2}=P$. Then $P$ is a projection matrix, projecting to the subspace of $H$ that is spanned by the vector $|A\rangle$. In fact, for any vector $|B\rangle$ we have

$$
P|B\rangle=|A\rangle\langle A|| B\rangle=|A\rangle\langle A \mid B\rangle=\langle A \mid B\rangle|A\rangle .
$$

If $\left\{\left|C_{1}\right\rangle,\left|C_{2}\right\rangle, \cdots\left|C_{n}\right\rangle\right\}$ is an orthonormal basis for $H$, and

$$
P_{i}=\left|C_{i}\right\rangle\left\langle C_{i}\right|
$$

then for any vector $|A\rangle$ we have

$$
|A\rangle=\left\langle C_{1} \mid A\right\rangle\left|C_{1}\right\rangle+\cdots+\left\langle C_{n} \mid A\right\rangle\left|C_{n}\right\rangle .
$$

Hence

$$
\langle B \mid A\rangle=\left\langle B \mid C_{1}\right\rangle\left\langle C_{1} \mid A\right\rangle+\cdots+\left\langle B \mid C_{n}\right\rangle\left\langle C_{n} \mid A\right\rangle
$$


One wants the probability of starting in state $|A\rangle$ and ending in state $|B\rangle$. The probability for this event is equal to $|\langle B \mid A\rangle|^{2}$. This can be refined if we have more knowledge. If the intermediate states $\left|C_{i}\right\rangle$ are a complete set of orthonormal alternatives then we can assume that $\left\langle C_{i} \mid C_{i}\right\rangle=1$ for each $i$ and that $\Sigma_{i}\left|C_{i}\right\rangle\left\langle C_{i}\right|=1$. This identity now corresponds to the fact that 1 is the sum of the probabilities of an arbitrary state being projected into one of these intermediate states.

If there are intermediate states between the intermediate states this formulation can be continued until one is summing over all possible paths from $A$ to $B$. This becomes the path integral expression for the amplitude $\langle B \mid A\rangle$.

\subsection{What is a Quantum Computer?}

A quantum computer is, abstractly, a composition $U$ of unitary transformations, together with an initial state and a choice of measurement basis. One runs the computer by repeatedly initializing it, and then measuring the result of applying the unitary transformation $U$ to the initial state. The results of these measurements are then analyzed for the desired information that the computer was set to determine. The key to using the computer is the design of the initial state and the design of the composition of unitary transformations. The reader should consult [71] for more specific examples of quantum algorithms.

Let $H$ be a given finite dimensional vector space over the complex numbers $C$. Let $\left\{W_{0}, W_{1}, \ldots, W_{n}\right\}$ be an orthonormal basis for $H$ so that with $|i\rangle:=\left|W_{i}\right\rangle$ denoting $W_{i}$ and $\langle i|$ denoting the conjugate transpose of $|i\rangle$, we have

$$
\langle i \mid j\rangle=\delta_{i j}
$$

where $\delta_{i j}$ denotes the Kronecker delta (equal to one when its indices are equal to one another, and equal to zero otherwise). Given a vector $v$ in $H$ let $|v|^{2}:=\langle v \mid v\rangle$. Note that $\langle i| v$ is the $i$-th coordinate of $v$.

An measurement of $v$ returns one of the coordinates $|i\rangle$ of $v$ with probability $\mid\left\langle i|v|^{2}\right.$. This model of measurement is a simple instance of the situation with a quantum mechanical system that is in a mixed state until it is observed. The result of observation is to put the system into one of the basis states.

When the dimension of the space $H$ is two $(n=1)$, a vector in the space is called a qubit. A qubit represents one quantum of binary information. On measurement, one obtains either the ket $|0\rangle$ or the ket $|1\rangle$. This constitutes 
the binary distinction that is inherent in a qubit. Note however that the information obtained is probabilistic. If the qubit is

$$
|\psi\rangle=\alpha|0\rangle+\beta|1\rangle,
$$

then the ket $|0\rangle$ is observed with probability $|\alpha|^{2}$, and the ket $|1\rangle$ is observed with probability $|\beta|^{2}$. In speaking of an idealized quantum computer, we do not specify the nature of measurement process beyond these probability postulates.

In the case of general dimension $n$ of the space $H$, we will call the vectors in $H$ qunits. It is quite common to use spaces $H$ that are tensor products of two-dimensional spaces (so that all computations are expressed in terms of qubits) but this is not necessary in principle. One can start with a given space, and later work out factorizations into qubit transformations.

A quantum computation consists in the application of a unitary transformation $U$ to an initial qunit $\psi=a_{0}|0\rangle+\ldots+a_{n}|n\rangle$ with $|\psi|^{2}=1$, plus an measurement of $U \psi$. A measurement of $U \psi$ returns the ket $|i\rangle$ with probability $\mid\left\langle i|U \psi|^{2}\right.$. In particular, if we start the computer in the state $\left.\mid i\right\rangle$, then the probability that it will return the state $|j\rangle$ is $|\langle j|U| i\rangle|^{2}$.

It is the necessity for writing a given computation in terms of unitary transformations, and the probabilistic nature of the result that characterizes quantum computation. Such computation could be carried out by an idealized quantum mechanical system. It is hoped that such systems can be physically realized.

\section{Braiding Operators and Universal Quantum Gates}

A class of invariants of knots and links called quantum invariants can be constructed by using representations of the Artin braid group, and more specifically by using solutions to the Yang-Baxter equation [10], first discovered in relation to $1+1$ dimensional quantum field theory, and 2 dimensional statistical mechanics. Braiding operators feature in constructing representations of the Artin braid group, and in the construction of invariants of knots and links.

A key concept in the construction of quantum link invariants is the association of a Yang-Baxter operator $R$ to each elementary crossing in a link diagram. The operator $R$ is a linear mapping

$$
R: V \otimes V \longrightarrow V \otimes V
$$


defined on the 2-fold tensor product of a vector space $V$, generalizing the permutation of the factors (i.e., generalizing a swap gate when $V$ represents one qubit). Such transformations are not necessarily unitary in topological applications. It is useful to understand when they can be replaced by unitary transformations for the purpose of quantum computing. Such unitary $R$-matrices can be used to make unitary representations of the Artin braid group.

A solution to the Yang-Baxter equation, as described in the last paragraph is a matrix $R$, regarded as a mapping of a two-fold tensor product of a vector space $V \otimes V$ to itself that satisfies the equation

$$
(R \otimes I)(I \otimes R)(R \otimes I)=(I \otimes R)(R \otimes I)(I \otimes R) .
$$

From the point of view of topology, the matrix $R$ is regarded as representing an elementary bit of braiding represented by one string crossing over another. In Figure 7 we have illustrated the braiding identity that corresponds to the YangBaxter equation. Each braiding picture with its three input lines (below) and output lines (above) corresponds to a mapping of the three fold tensor product of the vector space $V$ to itself, as required by the algebraic equation quoted above. The pattern of placement of the crossings in the diagram corresponds to the factors $R \otimes I$ and $I \otimes R$. This crucial topological move has an algebraic expression in terms of such a matrix $R$. Our approach in this section to relate topology, quantum computing, and quantum entanglement is through the use of the Yang-Baxter equation. In order to accomplish this aim, we need to study solutions of the Yang-Baxter equation that are unitary. Then the $R$ matrix can be seen either as a braiding matrix or as a quantum gate in a quantum computer.

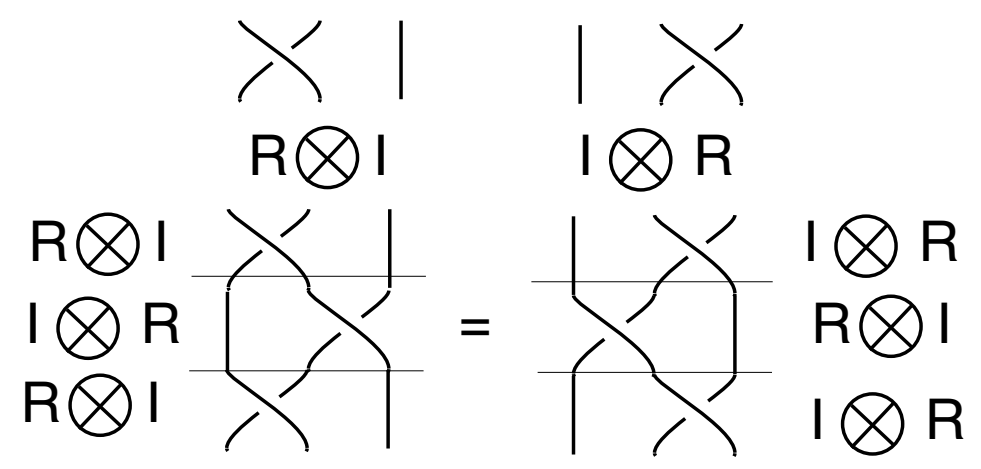

Figure 7 The Yang-Baxter equation $(R \otimes I)(I \otimes R)(R \otimes I)=(I \otimes R)(R \otimes I)(I \otimes R)$. 
The problem of finding solutions to the Yang-Baxter equation that are unitary turns out to be surprisingly difficult. Dye [25] has classified all such matrices of size $4 \times 4$. A rough summary of her classification is that all $4 \times$ 4 unitary solutions to the Yang-Baxter equation are similar to one of the following types of matrix:

$$
\begin{gathered}
R=\left(\begin{array}{cccc}
1 / \sqrt{2} & 0 & 0 & 1 / \sqrt{2} \\
0 & 1 / \sqrt{2} & -1 / \sqrt{2} & 0 \\
0 & 1 / \sqrt{2} & 1 / \sqrt{2} & 0 \\
-1 / \sqrt{2} & 0 & 0 & 1 / \sqrt{2}
\end{array}\right) \\
R^{\prime}=\left(\begin{array}{cccc}
a & 0 & 0 & 0 \\
0 & 0 & b & 0 \\
0 & c & 0 & 0 \\
0 & 0 & 0 & d
\end{array}\right) \\
R^{\prime \prime}=\left(\begin{array}{cccc}
0 & 0 & 0 & a \\
0 & b & 0 & 0 \\
0 & 0 & c & 0 \\
d & 0 & 0 & 0
\end{array}\right)
\end{gathered}
$$

where $a, b, c, d$ are unit complex numbers.

For the purpose of quantum computing, one should regard each matrix as acting on the stamdard basis $\{|00\rangle,|01\rangle,|10\rangle,|11\rangle\}$ of $H=V \otimes V$, where $V$ is a two-dimensional complex vector space. Then, for example we have

$$
\begin{gathered}
R|00\rangle=(1 / \sqrt{2})|00\rangle-(1 / \sqrt{2})|11\rangle, \\
R|01\rangle=(1 / \sqrt{2})|01\rangle+(1 / \sqrt{2})|10\rangle, \\
R|10\rangle=-(1 / \sqrt{2})|01\rangle+(1 / \sqrt{2})|10\rangle, \\
R|11\rangle=(1 / \sqrt{2})|00\rangle+(1 / \sqrt{2})|11\rangle .
\end{gathered}
$$

The reader should note that $R$ is the familiar change-of-basis matrix from the standard basis to the Bell basis of entangled states.

In the case of $R^{\prime}$, we have

$$
\begin{aligned}
& R^{\prime}|00\rangle=a|00\rangle, R^{\prime}|01\rangle=c|10\rangle, \\
& R^{\prime}|10\rangle=b|01\rangle, R^{\prime}|11\rangle=d|11\rangle .
\end{aligned}
$$


Note that $R^{\prime}$ can be regarded as a diagonal phase gate $P$, composed with a swap gate $S$.

$$
\begin{aligned}
& P=\left(\begin{array}{llll}
a & 0 & 0 & 0 \\
0 & b & 0 & 0 \\
0 & 0 & c & 0 \\
0 & 0 & 0 & d
\end{array}\right) \\
& S=\left(\begin{array}{llll}
1 & 0 & 0 & 0 \\
0 & 0 & 1 & 0 \\
0 & 1 & 0 & 0 \\
0 & 0 & 0 & 1
\end{array}\right)
\end{aligned}
$$

Compositions of solutions of the (Braiding) Yang-Baxter equation with the swap gate $S$ are called solutions to the algebraic Yang-Baxter equation. Thus the diagonal matrix $P$ is a solution to the algebraic Yang-Baxter equation.

Remark. Another avenue related to unitary solutions to the Yang-Baxter equation as quantum gates comes from using extra physical parameters in this equation (the rapidity parameter) that are related to statistical physics. In [90] we discovered that solutions to the Yang-Baxter equation with the rapidity parameter allow many new unitary solutions. The significance of these gates for quatnum computing is still under investigation.

\subsection{Universal Gates}

A two-qubit gate $G$ is a unitary linear mapping $G: V \otimes V \longrightarrow V$ where $V$ is a two complex dimensional vector space. We say that the gate $G$ is universal for quantum computation (or just universal) if $G$ together with local unitary transformations (unitary transformations from $V$ to $V$ ) generates all unitary transformations of the complex vector space of dimension $2^{n}$ to itself. It is wellknown [71] that CNOT is a universal gate. (On the standard basis, CNOT is the identity when the first qubit is 0 , and it flips the second qbit, leaving the first alone, when the first qubit is 1.)

A gate $G$, as above, is said to be entangling if there is a vector

$$
|\alpha \beta\rangle=|\alpha\rangle \otimes|\beta\rangle \in V \otimes V
$$

such that $G|\alpha \beta\rangle$ is not decomposable as a tensor product of two qubits. Under these circumstances, one says that $G|\alpha \beta\rangle$ is entangled. 
In [17], the Brylinskis give a general criterion of $G$ to be universal. They prove that a two-qubit gate $G$ is universal if and only if it is entangling.

Remark. A two-qubit pure state

$$
|\phi\rangle=a|00\rangle+b|01\rangle+c|10\rangle+d|11\rangle
$$

is entangled exactly when $(a d-b c) \neq 0$. It is easy to use this fact to check when a specific matrix is, or is not, entangling.

Remark. There are many gates other than CNOT that can be used as universal gates in the presence of local unitary transformations. Some of these are themselves topological (unitary solutions to the Yang-Baxter equation, see [56]) and themselves generate representations of the Artin braid group. Replacing CNOT by a solution to the Yang-Baxter equation does not place the local unitary transformations as part of the corresponding representation of the braid group. Thus such substitutions give only a partial solution to creating topological quantum computation. In this paper we are concerned with braid group representations that include all aspects of the unitary group. Accordingly, in the next section we shall first examine how the braid group on three strands can be represented as local unitary transformations.

Theorem. Let $D$ denote the phase gate shown below. $D$ is a solution to the algebraic Yang-Baxter equation (see the earlier discussion in this section). Then $D$ is a universal gate.

$$
D=\left(\begin{array}{cccc}
1 & 0 & 0 & 0 \\
0 & 1 & 0 & 0 \\
0 & 0 & 1 & 0 \\
0 & 0 & 0 & -1
\end{array}\right)
$$

Proof. It follows at once from the Brylinski Theorem that $D$ is universal. For a more specific proof, note that $C N O T=Q D Q^{-1}$, where $Q=H \otimes I, H$ is the $2 \times 2$ Hadamard matrix. The conclusion then follows at once from this identity and the discussion above. We illustrate the matrices involved in this proof below:

$$
\begin{gathered}
H=(1 / \sqrt{2})\left(\begin{array}{cc}
1 & 1 \\
1 & -1
\end{array}\right) \\
Q=(1 / \sqrt{2})\left(\begin{array}{cccc}
1 & 1 & 0 & 0 \\
1 & -1 & 0 & 0 \\
0 & 0 & 1 & 1 \\
0 & 0 & 1 & -1
\end{array}\right)
\end{gathered}
$$




$$
\begin{gathered}
D=\left(\begin{array}{cccc}
1 & 0 & 0 & 0 \\
0 & 1 & 0 & 0 \\
0 & 0 & 1 & 0 \\
0 & 0 & 0 & -1
\end{array}\right) \\
Q D Q^{-1}=Q D Q=\left(\begin{array}{cccc}
1 & 0 & 0 & 0 \\
0 & 1 & 0 & 0 \\
0 & 0 & 0 & 1 \\
0 & 0 & 1 & 0
\end{array}\right)=C N O T
\end{gathered}
$$

This completes the proof of the Theorem.

Remark. We thank Martin Roetteles [77] for pointing out the specific factorization of CNOT used in this proof.

Theorem. The matrix solutions $R^{\prime}$ and $R^{\prime \prime}$ to the Yang-Baxter equation, described above, are universal gates exactly when $a d-b c \neq 0$ for their internal parameters $a, b, c, d$. In particular, let $R_{0}$ denote the solution $R^{\prime}$ (above) to the Yang-Baxter equation with $a=b=c=1, d=-1$.

$$
\begin{aligned}
R^{\prime} & =\left(\begin{array}{llll}
a & 0 & 0 & 0 \\
0 & 0 & b & 0 \\
0 & c & 0 & 0 \\
0 & 0 & 0 & d
\end{array}\right) \\
R_{0} & =\left(\begin{array}{cccc}
1 & 0 & 0 & 0 \\
0 & 0 & 1 & 0 \\
0 & 1 & 0 & 0 \\
0 & 0 & 0 & -1
\end{array}\right)
\end{aligned}
$$

Then $R_{0}$ is a universal gate.

Proof. The first part follows at once from the Brylinski Theorem. In fact, letting $H$ be the Hadamard matrix as before, and

$$
\begin{gathered}
\sigma=\left(\begin{array}{cc}
1 / \sqrt{2} & i / \sqrt{2} \\
i / \sqrt{2} & 1 / \sqrt{2}
\end{array}\right), \lambda=\left(\begin{array}{cc}
1 / \sqrt{2} & 1 / \sqrt{2} \\
i / \sqrt{2} & -i / \sqrt{2}
\end{array}\right) \\
\mu=\left(\begin{array}{cc}
(1-i) / 2 & (1+i) / 2 \\
(1-i) / 2 & (-1-i) / 2
\end{array}\right) .
\end{gathered}
$$

Then

$$
C N O T=(\lambda \otimes \mu)\left(R_{0}(I \otimes \sigma) R_{0}\right)(H \otimes H) .
$$

This gives an explicit expression for CNOT in terms of $R_{0}$ and local unitary transformations (for which we thank Ben Reichardt). 
Remark. Let $S W A P$ denote the Yang-Baxter Solution $R^{\prime}$ with $a=b=c=$ $d=1$.

$$
S W A P=\left(\begin{array}{cccc}
1 & 0 & 0 & 0 \\
0 & 0 & 1 & 0 \\
0 & 1 & 0 & 0 \\
0 & 0 & 0 & 1
\end{array}\right)
$$

$S W A P$ is the standard swap gate. Note that $S W A P$ is not a universal gate. This also follows from the Brylinski Theorem, since $S W A P$ is not entangling. Note also that $R_{0}$ is the composition of the phase gate $D$ with this swap gate.

Theorem. Let

$$
R=\left(\begin{array}{cccc}
1 / \sqrt{2} & 0 & 0 & 1 / \sqrt{2} \\
0 & 1 / \sqrt{2} & -1 / \sqrt{2} & 0 \\
0 & 1 / \sqrt{2} & 1 / \sqrt{2} & 0 \\
-1 / \sqrt{2} & 0 & 0 & 1 / \sqrt{2}
\end{array}\right)
$$

be the unitary solution to the Yang-Baxter equation discussed above. Then $R$ is a universal gate. The proof below gives a specific expression for CNOT in terms of $R$.

Proof. This result follows at once from the Brylinksi Theorem, since $R$ is highly entangling. For a direct computational proof, it suffices to show that $C N O T$ can be generated from $R$ and local unitary transformations. Let

$$
\begin{gathered}
\alpha=\left(\begin{array}{cc}
1 / \sqrt{2} & 1 / \sqrt{2} \\
1 / \sqrt{2} & -1 / \sqrt{2}
\end{array}\right) \\
\beta=\left(\begin{array}{cc}
-1 / \sqrt{2} & 1 / \sqrt{2} \\
i / \sqrt{2} & i / \sqrt{2}
\end{array}\right) \\
\gamma=\left(\begin{array}{cc}
1 / \sqrt{2} & i / \sqrt{2} \\
1 / \sqrt{2} & -i / \sqrt{2}
\end{array}\right) \\
\delta=\left(\begin{array}{cc}
-1 & 0 \\
0 & -i
\end{array}\right)
\end{gathered}
$$

Let $M=\alpha \otimes \beta$ and $N=\gamma \otimes \delta$. Then it is straightforward to verify that

$$
C N O T=M R N .
$$

This completes the proof.

Remark. See [56] for more information about these calculations. 


\section{A Remark about $E P R$, Engtanglement and Bell's Inequality}

A state $|\psi\rangle \in H^{\otimes n}$, where $H$ is the qubit space, is said to be entangled if it cannot be written as a tensor product of vectors from non-trivial factors of $H^{\otimes n}$. Such states turn out to be related to subtle nonlocality in quantum physics. It helps to place this algebraic structure in the context of a gedanken experiment to see where the physics comes in. Thought experiments of the sort we are about to describe were first devised by Einstein, Podolosky and Rosen, referred henceforth as EPR.

Consider the entangled state

$$
S=(|0\rangle|1\rangle+|1\rangle|0\rangle) / \sqrt{2}
$$

In an EPR thought experiment, we think of two "parts" of this state that are separated in space. We want a notation for these parts and suggest the following:

$$
\begin{aligned}
& L=(\{|0\rangle\}|1\rangle+\{|1\rangle\}|0\rangle) / \sqrt{2}, \\
& R=(|0\rangle\{|1\rangle\}+|1\rangle\{|0\rangle\}) / \sqrt{2} .
\end{aligned}
$$

In the left state $L$, an observer can only observe the left hand factor. In the right state $R$, an observer can only observe the right hand factor. These "states" $L$ and $R$ together comprise the EPR state $S$, but they are accessible individually just as are the two photons in the usual thought experiement. One can transport $L$ and $R$ individually and we shall write

$$
S=L * R
$$

to denote that they are the "parts" (but not tensor factors) of $S$.

The curious thing about this formalism is that it includes a little bit of macroscopic physics implicitly, and so it makes it a bit more apparent what EPR were concerned about. After all, lots of things that we can do to $L$ or $R$ do not affect $S$. For example, transporting $L$ from one place to another, as in the original experiment where the photons separate. On the other hand, if Alice has $L$ and Bob has $R$ and Alice performs a local unitary transformation on "her" tensor factor, this applies to both $L$ and $R$ since the transformation is actually being applied to the state $S$. This is also a "spooky action at a distance" whose consequence does not appear until a measurement is made. 
To go a bit deeper it is worthwhile seeing what entanglement, in the sense of tensor indecomposability, has to do with the structure of the EPR thought experiment. To this end, we look at the structure of the Bell inequalities using the Clauser, Horne, Shimony, Holt formalism $(\mathrm{CHSH})$ as explained in the book by Nielsen and Chuang [71]. For this we use the following observables with eigenvalues \pm 1 .

$$
\begin{gathered}
Q=\left(\begin{array}{cc}
1 & 0 \\
0 & -1
\end{array}\right)_{1}, \\
R=\left(\begin{array}{ll}
0 & 1 \\
1 & 0
\end{array}\right)_{1}, \\
S=\left(\begin{array}{cc}
-1 & -1 \\
-1 & 1
\end{array}\right)_{2} / \sqrt{2}, \\
T=\left(\begin{array}{cc}
1 & -1 \\
-1 & -1
\end{array}\right)_{2} / \sqrt{2} .
\end{gathered}
$$

The subscripts 1 and 2 on these matrices indicate that they are to operate on the first and second tensor factors, repsectively, of a quantum state of the form

$$
\phi=a|00\rangle+b|01\rangle+c|10\rangle+d|11\rangle
$$

To simplify the results of this calculation we shall here assume that the coefficients $a, b, c, d$ are real numbers. We calculate the quantity

$$
\Delta=\langle\phi|Q S| \phi\rangle+\langle\phi|R S| \phi\rangle+\langle\phi|R T| \phi\rangle-\langle\phi|Q T| \phi\rangle,
$$

finding that

$$
\Delta=\left(2-4(a+d)^{2}+4(a d-b c)\right) / \sqrt{2} .
$$

Classical probability calculation with random variables of value \pm 1 gives the value of $Q S+R S+R T-Q T= \pm 2$ (with each of $Q, R, S$ and $T$ equal to \pm 1 ). Hence the classical expectation satisfies the Bell inequality

$$
E(Q S)+E(R S)+E(R T)-E(Q T) \leq 2
$$

That quantum expectation is not classical is embodied in the fact that $\Delta$ can be greater than 2. The classic case is that of the Bell state

$$
\phi=(|01\rangle-|10\rangle) / \sqrt{2}
$$

Here

$$
\Delta=6 / \sqrt{2}>2
$$


In general we see that the following inequality is needed in order to violate the Bell inequality

$$
\left(2-4(a+d)^{2}+4(a d-b c)\right) / \sqrt{2}>2 .
$$

This is equivalent to

$$
(\sqrt{2}-1) / 2<(a d-b c)-(a+d)^{2} .
$$

Since we know that $\phi$ is entangled exactly when $a d-b c$ is non-zero, this shows that an unentangled state cannot violate the Bell inequality. This formula also shows that it is possible for a state to be entangled and yet not violate the Bell inequality. For example, if

$$
\phi=(|00\rangle-|01\rangle+|10\rangle+|11\rangle) / 2,
$$

then $\Delta(\phi)$ satisfies Bell's inequality, but $\phi$ is an entangled state. We see from this calculation that entanglement in the sense of tensor indecomposability, and entanglement in the sense of Bell inequality violation for a given choice of Bell operators are not equivalent concepts. On the other hand, Benjamin Schumacher has pointed out [79] that any entangled two-qubit state will violate Bell inequalities for an appropriate choice of operators. This deepens the context for our question of the relationship between topological entanglement and quantum entanglement. The Bell inequality violation is an indication of quantum mechanical entanglement. One's intuition suggests that it is this sort of entanglement that should have a topological context.

\section{The Aravind Hypothesis}

Link diagrams can be used as graphical devices and holders of information. In this vein Aravind [5] proposed that the entanglement of a link should correspond to the entanglement of a state. Measurement of a link would be modeled by deleting one component of the link. A key example is the Borromean rings. See Figure 8.

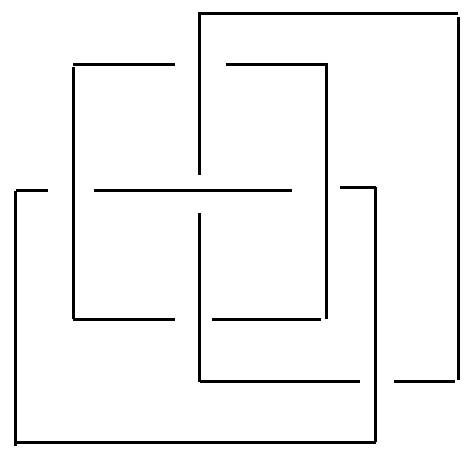




\section{Figure 8 - Borromean Rings}

Deleting any component of the Boromean rings yields a remaining pair of unlinked rings. The Borromean rings are entangled, but any two of them are unentangled. In this sense the Borromean rings are analogous to the $G H Z$ state $|G H Z\rangle=(1 / \sqrt{2})(|000\rangle+|111\rangle)$. Measurement in any factor of the $G H Z$ yields an unentangled state. Aravind points out that this property is basis dependent. We point out that there are states whose entanglement after an measurement is a matter of probability (via quantum amplitudes). Consider for example the state

$$
|\psi\rangle=|001\rangle+|010\rangle+|100\rangle
$$

Measurement in any coordinate yields an entangled or an unentangled state with equal probability. For example

$$
|\psi\rangle=|0\rangle(|01\rangle+|10\rangle)+|1\rangle|00\rangle
$$

so that projecting to $|1\rangle$ in the first coordinate yields an unentangled state, while projecting to $|0\rangle$ yields an entangled state, each with equal probability.

New ways to use link diagrams must be invented to map the properties of such states. One direction is to consider appropriate notions of quantum knots so that one can formlate superpositions of topological types as in [55]. But one needs to go deeper in this consideration. The relationship of topology and physics needs to be examined carefully. We take the stance that topological properties of systems are properties that remain invariant under certain transformations that are identified as "topological equivalences". In making quantum physical models, these equivalences should correspond to unitary transformations of an appropriate Hilbert space. Accordingly, we have formulated a model for quantum knots [60] that meets these requirements. A quantum knot system represents the "quantum embodiment" of a closed knotted physical piece of rope. A quantum knot (i.e., an element $|K\rangle$ lying in an appropriate Hilbert space $H_{n}$, as a state of this system, represents the state of such a knotted closed piece of rope, i.e., the particular spatial configuration of the knot tied in the rope. Associated with a quantum knot system is a group of unitary transformations $A_{n}$, called the ambient group, which represents all possible ways of moving the rope around (without cutting the rope, and without letting the rope pass through itself.) Of course, unlike a classical closed piece of rope, a quantum knot can exhibit non-classical behavior, such as quantum 
superposition and quantum entanglement. The knot type of a quantum knot $|K\rangle$ is simply the orbit of the quantum knot under the action of the ambient group $A_{n}$. This leads to new questions connecting quantum computing and knot theory.

\section{$6 S U(2)$ Representations of the Artin Braid Group}

The purpose of this section is to determine all the representations of the three strand Artin braid group $B_{3}$ to the special unitary group $S U(2)$ and concomitantly to the unitary group $U(2)$. One regards the groups $S U(2)$ and $U(2)$ as acting on a single qubit, and so $U(2)$ is usually regarded as the group of local unitary transformations in a quantum information setting. If one is looking for a coherent way to represent all unitary transformations by way of braids, then $U(2)$ is the place to start. Here we will show that there are many representations of the three-strand braid group that generate a dense subset of $U(2)$. Thus it is a fact that local unitary transformations can be "generated by braids" in many ways.

We begin with the structure of $S U(2)$. A matrix in $S U(2)$ has the form

$$
M=\left(\begin{array}{cc}
z & w \\
-\bar{w} & \bar{z}
\end{array}\right)
$$

where $z$ and $w$ are complex numbers, and $\bar{z}$ denotes the complex conjugate of $z$. To be in $S U(2)$ it is required that $\operatorname{Det}(M)=1$ and that $M^{\dagger}=M^{-1}$ where Det denotes determinant, and $M^{\dagger}$ is the conjugate transpose of $M$. Thus if $z=a+b i$ and $w=c+d i$ where $a, b, c, d$ are real numbers, and $i^{2}=-1$, then

$$
M=\left(\begin{array}{cc}
a+b i & c+d i \\
-c+d i & a-b i
\end{array}\right)
$$

with $a^{2}+b^{2}+c^{2}+d^{2}=1$. It is convenient to write

$$
M=a\left(\begin{array}{ll}
1 & 0 \\
0 & 1
\end{array}\right)+b\left(\begin{array}{cc}
i & 0 \\
0 & -i
\end{array}\right)+c\left(\begin{array}{cc}
0 & 1 \\
-1 & 0
\end{array}\right)+d\left(\begin{array}{cc}
0 & i \\
i & 0
\end{array}\right)
$$

and to abbreviate this decomposition as

$$
M=a+b i+c j+d k
$$


where

$$
1 \equiv\left(\begin{array}{cc}
1 & 0 \\
0 & 1
\end{array}\right), i \equiv\left(\begin{array}{cc}
i & 0 \\
0 & -i
\end{array}\right), j \equiv,\left(\begin{array}{cc}
0 & 1 \\
-1 & 0
\end{array}\right), k \equiv\left(\begin{array}{cc}
0 & i \\
i & 0
\end{array}\right)
$$

so that

$$
i^{2}=j^{2}=k^{2}=i j k=-1
$$

and

$$
\begin{gathered}
i j=k, j k=i, k i=j \\
j i=-k, k j=-i, i k=-j .
\end{gathered}
$$

The algebra of $1, i, j, k$ is called the quaternions after William Rowan Hamilton who discovered this algebra prior to the discovery of matrix algebra. Thus the unit quaternions are identified with $S U(2)$ in this way. We shall use this identification, and some facts about the quaternions to find the $S U(2)$ representations of braiding. First we recall some facts about the quaternions.

1. Note that if $q=a+b i+c j+d k$ (as above), then $q^{\dagger}=a-b i-c j-d k$ so that $q q^{\dagger}=a^{2}+b^{2}+c^{2}+d^{2}=1$.

2. A general quaternion has the form $q=a+b i+c j+d k$ where the value of $q q^{\dagger}=a^{2}+b^{2}+c^{2}+d^{2}$, is not fixed to unity. The length of $q$ is by definition $\sqrt{q q^{\dagger}}$.

3. A quaternion of the form $r i+s j+t k$ for real numbers $r, s, t$ is said to be a pure quaternion. We identify the set of pure quaternions with the vector space of triples $(r, s, t)$ of real numbers $R^{3}$.

4. Thus a general quaternion has the form $q=a+b u$ where $u$ is a pure quaternion of unit length and $a$ and $b$ are arbitrary real numbers. A unit quaternion (element of $S U(2)$ ) has the addition property that $a^{2}+b^{2}=1$.

5. If $u$ is a pure unit length quaternion, then $u^{2}=-1$. Note that the set of pure unit quaternions forms the two-dimensional sphere $S^{2}=$ $\left\{(r, s, t) \mid r^{2}+s^{2}+t^{2}=1\right\}$ in $R^{3}$.

6. If $u, v$ are pure quaternions, then

$$
u v=-u \cdot v+u \times v
$$

whre $u \cdot v$ is the dot product of the vectors $u$ and $v$, and $u \times v$ is the vector cross product of $u$ and $v$. In fact, one can take the definition of quaternion multiplication as

$$
(a+b u)(c+d v)=a c+b c(u)+a d(v)+b d(-u \cdot v+u \times v),
$$


and all the above properties are consequences of this definition. Note that quaternion multiplication is associative.

7. Let $g=a+b u$ be a unit length quaternion so that $u^{2}=-1$ and $a=$ $\cos (\theta / 2), b=\sin (\theta / 2)$ for a chosen angle $\theta$. Define $\phi_{g}: R^{3} \longrightarrow R^{3}$ by the equation $\phi_{g}(P)=g P g^{\dagger}$, for $P$ any point in $R^{3}$, regarded as a pure quaternion. Then $\phi_{g}$ is an orientation preserving rotation of $R^{3}$ (hence an element of the rotation group $S O(3))$. Specifically, $\phi_{g}$ is a rotation about the axis $u$ by the angle $\theta$. The mapping

$$
\phi: S U(2) \longrightarrow S O(3)
$$

is a two-to-one surjective map from the special unitary group to the rotation group. In quaternionic form, this result was proved by Hamilton and by Rodrigues in the middle of the nineteeth century. The specific formula for $\phi_{g}(P)$ as shown below:

$$
\phi_{g}(P)=g P g^{-1}=\left(a^{2}-b^{2}\right) P+2 a b(P \times u)+2(P \cdot u) b^{2} u .
$$

We want a representation of the three-strand braid group in $S U(2)$. This means that we want a homomorphism $\rho: B_{3} \longrightarrow S U(2)$, and hence we want elements $g=\rho\left(s_{1}\right)$ and $h=\rho\left(s_{2}\right)$ in $S U(2)$ representing the braid group generators $s_{1}$ and $s_{2}$. Since $s_{1} s_{2} s_{1}=s_{2} s_{1} s_{2}$ is the generating relation for $B_{3}$, the only requirement on $g$ and $h$ is that $g h g=h g h$. We rewrite this relation as $h^{-1} g h=g h g^{-1}$, and analyze its meaning in the unit quaternions.

Suppose that $g=a+b u$ and $h=c+d v$ where $u$ and $v$ are unit pure quaternions so that $a^{2}+b^{2}=1$ and $c^{2}+d^{2}=1$. then $g h g^{-1}=c+d \phi_{g}(v)$ and $h^{-1} g h=a+b \phi_{h^{-1}}(u)$. Thus it follows from the braiding relation that $a=c$, $b= \pm d$, and that $\phi_{g}(v)= \pm \phi_{h^{-1}}(u)$. However, in the case where there is a minus sign we have $g=a+b u$ and $h=a-b v=a+b(-v)$. Thus we can now prove the following Theorem.

Theorem. If $g=a+b u$ and $h=c+d v$ are pure unit quaternions,then, without loss of generality, the braid relation $g h g=h g h$ is true if and only if $h=a+b v$, and $\phi_{g}(v)=\phi_{h^{-1}}(u)$. Furthermore, given that $g=a+b u$ and $h=a+b v$, the condition $\phi_{g}(v)=\phi_{h^{-1}}(u)$ is satisfied if and only if $u \cdot v=\frac{a^{2}-b^{2}}{2 b^{2}}$ when $u \neq v$. If $u=v$ then then $g=h$ and the braid relation is trivially satisfied.

Proof. We have proved the first sentence of the Theorem in the discussion prior to its statement. Therefore assume that $g=a+b u, h=a+b v$, and 
$\phi_{g}(v)=\phi_{h^{-1}}(u)$. We have already stated the formula for $\phi_{g}(v)$ in the discussion about quaternions:

$$
\phi_{g}(v)=g v g^{-1}=\left(a^{2}-b^{2}\right) v+2 a b(v \times u)+2(v \cdot u) b^{2} u .
$$

By the same token, we have

$$
\begin{gathered}
\phi_{h^{-1}}(u)=h^{-1} u h=\left(a^{2}-b^{2}\right) u+2 a b(u \times-v)+2(u \cdot(-v)) b^{2}(-v) \\
=\left(a^{2}-b^{2}\right) u+2 a b(v \times u)+2(v \cdot u) b^{2}(v) .
\end{gathered}
$$

Hence we require that

$$
\left(a^{2}-b^{2}\right) v+2(v \cdot u) b^{2} u=\left(a^{2}-b^{2}\right) u+2(v \cdot u) b^{2}(v) .
$$

This equation is equivalent to

$$
2(u \cdot v) b^{2}(u-v)=\left(a^{2}-b^{2}\right)(u-v) .
$$

If $u \neq v$, then this implies that

$$
u \cdot v=\frac{a^{2}-b^{2}}{2 b^{2}} .
$$

This completes the proof of the Theorem.

An Example. Let

$$
g=e^{i \theta}=a+b i
$$

where $a=\cos (\theta)$ and $b=\sin (\theta)$. Let

$$
h=a+b\left[\left(c^{2}-s^{2}\right) i+2 c s k\right]
$$

where $c^{2}+s^{2}=1$ and $c^{2}-s^{2}=\frac{a^{2}-b^{2}}{2 b^{2}}$. Then we can rewrite $g$ and $h$ in matrix form as the matrices $G$ and $H$. Instead of writing the explicit form of $H$, we write $H=F G F^{\dagger}$ where $F$ is an element of $S U(2)$ as shown below.

$$
\begin{aligned}
& G=\left(\begin{array}{cc}
e^{i \theta} & 0 \\
0 & e^{-i \theta}
\end{array}\right) \\
& F=\left(\begin{array}{cc}
i c & i s \\
i s & -i c
\end{array}\right)
\end{aligned}
$$

This representation of braiding where one generator $G$ is a simple matrix of phases, while the other generator $H=F G F^{\dagger}$ is derived from $G$ by conjugation by a unitary matrix, has the possibility for generalization to representations of braid groups (on greater than three strands) to $S U(n)$ or $U(n)$ for $n$ greater 
than 2. In fact we shall see just such representations constructed later in this paper, by using a version of topological quantum field theory. The simplest example is given by

$$
\begin{gathered}
g=e^{7 \pi i / 10} \\
f=i \tau+k \sqrt{\tau} \\
h=f r f^{-1}
\end{gathered}
$$

where $\tau^{2}+\tau=1$. Then $g$ and $h$ satisfy $g h g=h g h$ and generate a representation of the three-strand braid group that is dense in $S U(2)$. We shall call this the Fibonacci representation of $B_{3}$ to $S U(2)$.

Density. Consider representations of $B_{3}$ into $S U(2)$ produced by the method of this section. That is consider the subgroup $S U[G, H]$ of $S U(2)$ generated by a pair of elements $\{g, h\}$ such that $g h g=h g h$. We wish to understand when such a representation will be dense in $S U(2)$. We need the following lemma.

Lemma. $e^{a i} e^{b j} e^{c i}=\cos (b) e^{i(a+c)}+\sin (b) e^{i(a-c)} j$. Hence any element of $S U(2)$ can be written in the form $e^{a i} e^{b j} e^{c i}$ for appropriate choices of angles $a, b, c$. In fact, if $u$ and $v$ are linearly independent unit vectors in $R^{3}$, then any element of $S U(2)$ can be written in the form

$$
e^{a u} e^{b v} e^{c u}
$$

for appropriate choices of the real numbers $a, b, c$.

Proof. It is easy to check that

$$
e^{a i} e^{b j} e^{c i}=\cos (b) e^{i(a+c)}+\sin (b) e^{i(a-c)} j .
$$

This completes the verification of the identity in the statement of the Lemma.

Let $v$ be any unit direction in $R^{3}$ and $\lambda$ an arbitrary angle. We have

$$
e^{v \lambda}=\cos (\lambda)+\sin (\lambda) v
$$

and

$$
v=r+s i+(p+q i) j
$$

where $r^{2}+s^{2}+p^{2}+q^{2}=1$. So

$$
\begin{gathered}
e^{v \lambda}=\cos (\lambda)+\sin (\lambda)[r+s i]+\sin (\lambda)[p+q i] j \\
=[(\cos (\lambda)+\sin (\lambda) r)+\sin (\lambda) \operatorname{si}]+[\sin (\lambda) p+\sin (\lambda) q i] j .
\end{gathered}
$$


By the identity just proved, we can choose angles $a, b, c$ so that

$$
e^{v \lambda}=e^{i a} e^{j b} e^{i c}
$$

Hence

$$
\cos (b) e^{i(a+c)}=(\cos (\lambda)+\sin (\lambda) r)+\sin (\lambda) s i
$$

and

$$
\sin (b) e^{i(a-c)}=\sin (\lambda) p+\sin (\lambda) q i
$$

Suppose we keep $v$ fixed and vary $\lambda$. Then the last equations show that this will result in a full variation of $b$.

Now consider

$$
e^{i a^{\prime}} e^{v \lambda} e^{i c^{\prime}}=e^{i a^{\prime}} e^{i a} e^{j b} e^{i c} e^{i b^{\prime}}=e^{i\left(a^{\prime}+a\right)} e^{j b} e^{i\left(c+c^{\prime}\right)} .
$$

By the basic identity, this shows that any element of $S U(2)$ can be written in the form

$$
e^{i a^{\prime}} e^{v \lambda} e^{i c^{\prime}}
$$

Then, by applying a rotation, we finally conclude that if $u$ and $v$ are linearly independent unit vectors in $R^{3}$, then any element of $S U(2)$ can be written in the form

$$
e^{a u} e^{b v} e^{c u}
$$

for appropriate choices of the real numbers $a, b, c$.

This Lemma can be used to verify the density of a representation, by finding two elements $A$ and $B$ in the representation such that the powers of $A$ are dense in the rotations about its axis, and the powers of $B$ are dense in the rotations about its axis, and such that the axes of $A$ and $B$ are linearly independent in $R^{3}$. Then by the Lemma the set of elements $A^{a+c} B^{b} A^{a-c}$ are dense in $S U(2)$. It follows for example, that the Fibonacci representation described above is dense in $S U(2)$, and indeed the generic representation of $B_{3}$ into $S U(2)$ will be dense in $S U(2)$. Our next task is to describe representations of the higher braid groups that will extend some of these unitary repressentations of the three-strand braid group. For this we need more topology.

\section{The Bracket Polynomial and the Jones Poly- nomial}

We now discuss the Jones polynomial. We shall construct the Jones polynomial by using the bracket state summation model [37]. The bracket polynomial, 
invariant under Reidmeister moves II and III, can be normalized to give an invariant of all three Reidemeister moves. This normalized invariant, with a change of variable, is the Jones polynomial $[35,36]$. The Jones polynomial was originally discovered by a different method than the one given here.

The bracket polynomial, $\langle K\rangle=\langle K\rangle(A)$, assigns to each unoriented link diagram $K$ a Laurent polynomial in the variable $A$, such that

1. If $K$ and $K^{\prime}$ are regularly isotopic diagrams, then $\langle K\rangle=\left\langle K^{\prime}\right\rangle$.

2. If $K \sqcup O$ denotes the disjoint union of $K$ with an extra unknotted and unlinked component $O$ (also called 'loop' or 'simple closed curve' or 'Jordan curve'), then

$$
<K \sqcup O>=\delta<K>\text {, }
$$

where

$$
\delta=-A^{2}-A^{-2} .
$$

3. $<K>$ satisfies the following formulas

$$
\begin{aligned}
& \left.<\chi>=A<\asymp>+A^{-1}<\right)(> \\
& \left.<\bar{\chi}>=A^{-1}<\asymp>+A<\right)(>,
\end{aligned}
$$

where the small diagrams represent parts of larger diagrams that are identical except at the site indicated in the bracket. We take the convention that the letter chi, $\chi$, denotes a crossing where the curved line is crossing over the straight segment. The barred letter denotes the switch of this crossing, where the curved line is undercrossing the straight segment. See Figure 9 for a graphic illustration of this relation, and an indication of the convention for choosing the labels $A$ and $A^{-1}$ at a given crossing. 


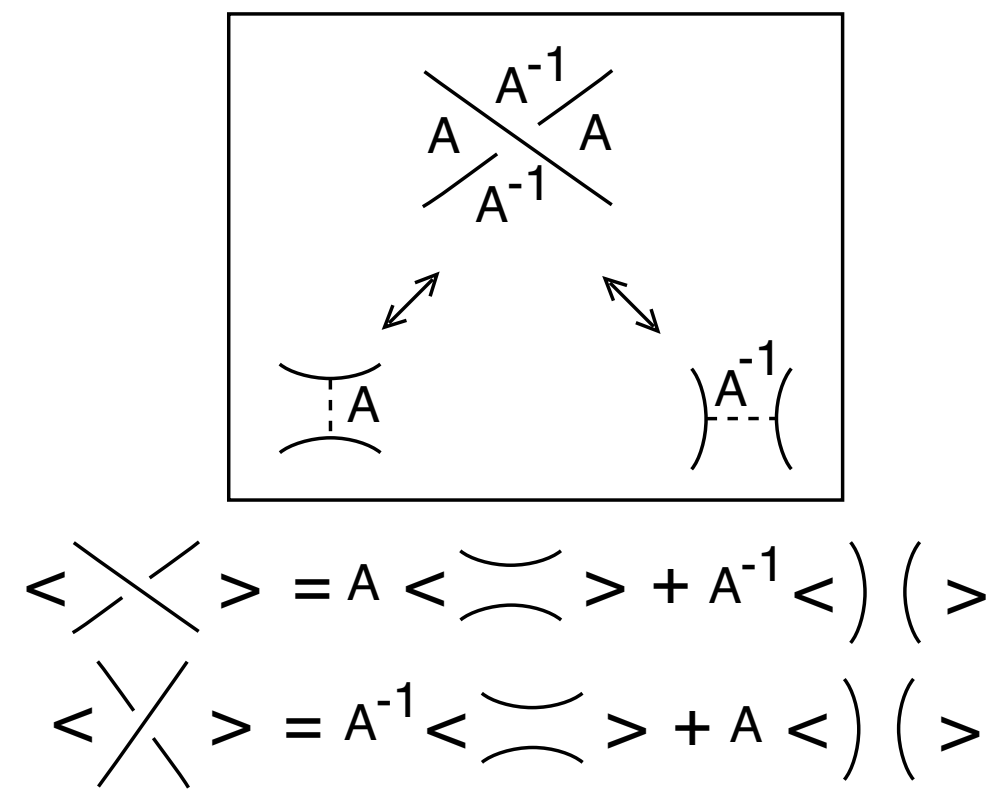

Figure 9 - Bracket Smoothings

It is easy to see that Properties 2 and 3 define the calculation of the bracket on arbitrary link diagrams. The choices of coefficients $\left(A\right.$ and $\left.A^{-1}\right)$ and the value of $\delta$ make the bracket invariant under the Reidemeister moves II and III. Thus Property 1 is a consequence of the other two properties.

In computing the bracket, one finds the following behaviour under Reidemeister move I:

$$
<\gamma>=-A^{3}<\smile>
$$

and

$$
<\bar{\gamma}>=-A^{-3}<\smile>
$$

where $\gamma$ denotes a curl of positive type as indicated in Figure 10, and $\bar{\gamma}$ indicates a curl of negative type, as also seen in this figure. The type of a curl is the sign of the crossing when we orient it locally. Our convention of signs is also given in Figure 10. Note that the type of a curl does not depend on the orientation we choose. The small arcs on the right hand side of these formulas indicate the removal of the curl from the corresponding diagram.

The bracket is invariant under regular isotopy and can be normalized to an invariant of ambient isotopy by the definition

$$
f_{K}(A)=\left(-A^{3}\right)^{-w(K)}<K>(A),
$$


where we chose an orientation for $K$, and where $w(K)$ is the sum of the crossing signs of the oriented link $K . w(K)$ is called the writhe of $K$. The convention for crossing signs is shown in Figure 10.
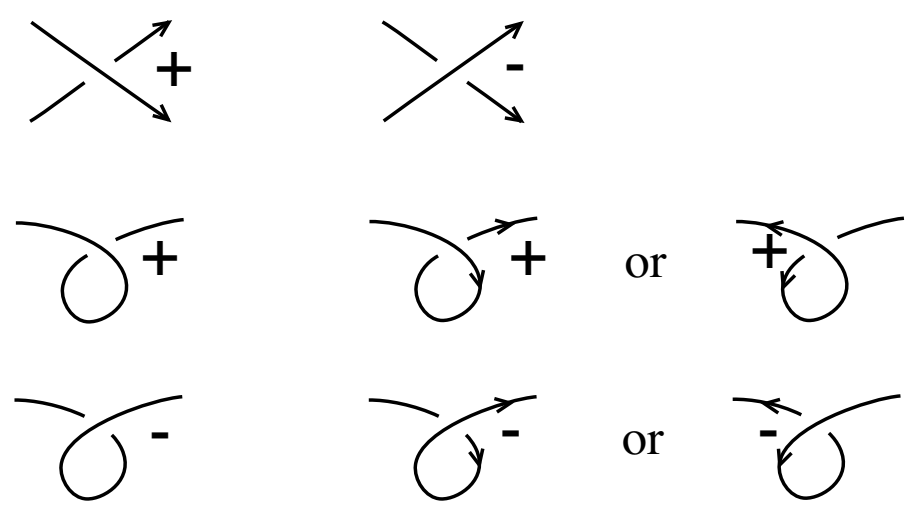

Figure 10 - Crossing Signs and Curls

One useful consequence of these formulas is the following switching formula

$$
A<\chi>-A^{-1}<\bar{\chi}>=\left(A^{2}-A^{-2}\right)<\asymp>.
$$

Note that in these conventions the $A$-smoothing of $\chi$ is $\asymp$, while the $A$ smoothing of $\bar{\chi}$ is )(. Properly interpreted, the switching formula above says that you can switch a crossing and smooth it either way and obtain a three diagram relation. This is useful since some computations will simplify quite quickly with the proper choices of switching and smoothing. Remember that it is necessary to keep track of the diagrams up to regular isotopy (the equivalence relation generated by the second and third Reidemeister moves). Here is an example. View Figure 11.
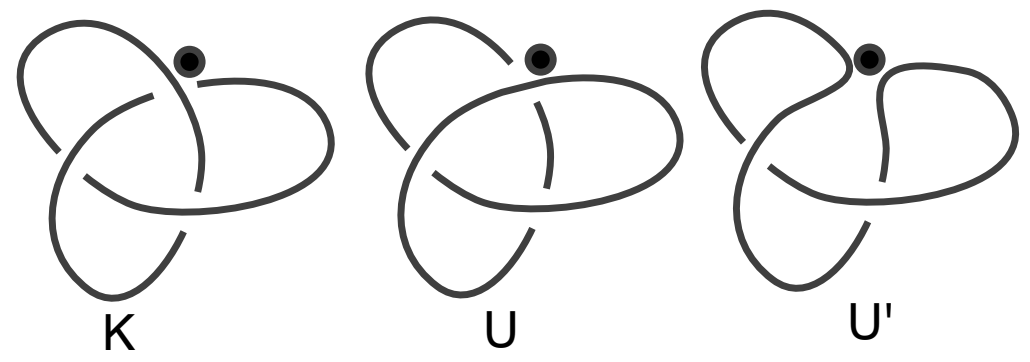

Figure 11 - Trefoil and Two Relatives 
Figure 11 shows a trefoil diagram $K$, an unknot diagram $U$ and another unknot diagram $U^{\prime}$. Applying the switching formula, we have

$$
A^{-1}<K>-A<U>=\left(A^{-2}-A^{2}\right)<U^{\prime}>
$$

and $\left\langle U>=-A^{3}\right.$ and $<U^{\prime}>=\left(-A^{-3}\right)^{2}=A^{-6}$. Thus

$$
A^{-1}<K>-A\left(-A^{3}\right)=\left(A^{-2}-A^{2}\right) A^{-6} .
$$

Hence

$$
A^{-1}<K>=-A^{4}+A^{-8}-A^{-4} .
$$

Thus

$$
<K>=-A^{5}-A^{-3}+A^{-7} .
$$

This is the bracket polynomial of the trefoil diagram $K$.

Since the trefoil diagram $K$ has writhe $w(K)=3$, we have the normalized polynomial

$$
f_{K}(A)=\left(-A^{3}\right)^{-3}<K>=-A^{-9}\left(-A^{5}-A^{-3}+A^{-7}\right)=A^{-4}+A^{-12}-A^{-16} .
$$

The bracket model for the Jones polynomial is quite useful both theoretically and in terms of practical computations. One of the neatest applications is to simply compute, as we have done, $f_{K}(A)$ for the trefoil knot $K$ and determine that $f_{K}(A)$ is not equal to $f_{K}\left(A^{-1}\right)=f_{-K}(A)$. This shows that the trefoil is not ambient isotopic to its mirror image, a fact that is much harder to prove by classical methods.

The State Summation. In order to obtain a closed formula for the bracket, we now describe it as a state summation. Let $K$ be any unoriented link diagram. Define a state, $S$, of $K$ to be a choice of smoothing for each crossing of $K$. There are two choices for smoothing a given crossing, and thus there are $2^{N}$ states of a diagram with $N$ crossings. In a state we label each smoothing with $A$ or $A^{-1}$ according to the left-right convention discussed in Property 3 (see Figure 9). The label is called a vertex weight of the state. There are two evaluations related to a state. The first one is the product of the vertex weights, denoted

$$
<K \mid S>\text {. }
$$

The second evaluation is the number of loops in the state $S$, denoted 
Define the state summation, $\langle K>$, by the formula

$$
<K>=\sum_{S}<K \mid S>\delta^{\|S\|-1}
$$

It follows from this definition that $\langle K\rangle$ satisfies the equations

$$
\begin{gathered}
\left.<\chi>=A<\asymp>+A^{-1}<\right)(>, \\
<K \sqcup O>=\delta<K> \\
<O>=1 .
\end{gathered}
$$

The first equation expresses the fact that the entire set of states of a given diagram is the union, with respect to a given crossing, of those states with an $A$-type smoothing and those with an $A^{-1}$-type smoothing at that crossing. The second and the third equation are clear from the formula defining the state summation. Hence this state summation produces the bracket polynomial as we have described it at the beginning of the section.

Remark. By a change of variables one obtains the original Jones polynomial, $V_{K}(t)$, for oriented knots and links from the normalized bracket:

$$
V_{K}(t)=f_{K}\left(t^{-\frac{1}{4}}\right)
$$

Remark. The bracket polynomial provides a connection between knot theory and physics, in that the state summation expression for it exhibits it as a generalized partition function defined on the knot diagram. Partition functions are ubiquitous in statistical mechanics, where they express the summation over all states of the physical system of probability weighting functions for the individual states. Such physical partition functions contain large amounts of information about the corresponding physical system. Some of this information is directly present in the properties of the function, such as the location of critical points and phase transition. Some of the information can be obtained by differentiating the partition function, or performing other mathematical operations on it.

There is much more in this connection with statistical mechanics in that the local weights in a partition function are often expressed in terms of solutions to a matrix equation called the Yang-Baxter equation, that turns out to fit perfectly invariance under the third Reidemeister move. As a result, there are many ways to define partition functions of knot diagrams that give rise to invariants of knots and links. The subject is intertwined with the algebraic structure of Hopf algebras and quantum groups, useful for producing systematic solutions to the Yang-Baxter equation. In fact Hopf algebras are deeply 
connected with the problem of constructing invariants of three-dimensional manifolds in relation to invariants of knots. We have chosen, in this survey paper, to not discuss the details of these approaches, but rather to proceed to Vassiliev invariants and the relationships with Witten's functional integral. The reader is referred to $[37,38,39,40,43,44,3,35,36,45,75,76,83,84]$ for more information about relationships of knot theory with statistical mechanics, Hopf algebras and quantum groups. For topology, the key point is that Lie algebras can be used to construct invariants of knots and links.

\subsection{Quantum Computation of the Jones Polynomial}

Can the invariants of knots and links such as the Jones polynomial be configured as quantum computers? This is an important question because the algorithms to compute the Jones polynomial are known to be $N P$-hard, and so corresponding quantum algorithms may shed light on the relationship of this level of computational complexity with quantum computing (See [29]). Such models can be formulated in terms of the Yang-Baxter equation [37, 38, 44, 49]. The next paragraph explains how this comes about.

In Figure 12, we indicate how topological braiding plus maxima (caps) and minima (cups) can be used to configure the diagram of a knot or link. This also can be translated into algebra by the association of a Yang-Baxter matrix $R$ (not necessarily the $R$ of the previous sections) to each crossing and other matrices to the maxima and minima. There are models of very effective invariants of knots and links such as the Jones polynomial that can be put into this form [49]. In this way of looking at things, the knot diagram can be viewed as a picture, with time as the vertical dimension, of particles arising from the vacuum, interacting (in a two-dimensional space) and finally annihilating one another. The invariant takes the form of an amplitude for this process that is computed through the association of the Yang-Baxter solution $R$ as the scattering matrix at the crossings and the minima and maxima as creation and annihilation operators. Thus we can write the amplitude in the form

$$
Z_{K}=\langle C U P|M| C A P\rangle
$$

where $\langle C U P|$ denotes the composition of cups, $M$ is the composition of elementary braiding matrices, and $|C A P\rangle$ is the composition of caps. We regard $\langle C U P|$ as the preparation of this state, and $|C A P\rangle$ as the measurement of this state. In order to view $Z_{K}$ as a quantum computation, $M$ must be a unitary operator. This is the case when the $R$-matrices (the solutions to the YangBaxter equation used in the model) are unitary. Each $R$-matrix is viewed as a 
quantum gate (or possibly a composition of quantum gates), and the vacuumvacuum diagram for the knot is interpreted as a quantum computer. This quantum computer will probabilistically (via quantum amplitudes) compute the values of the states in the state sum for $Z_{K}$.

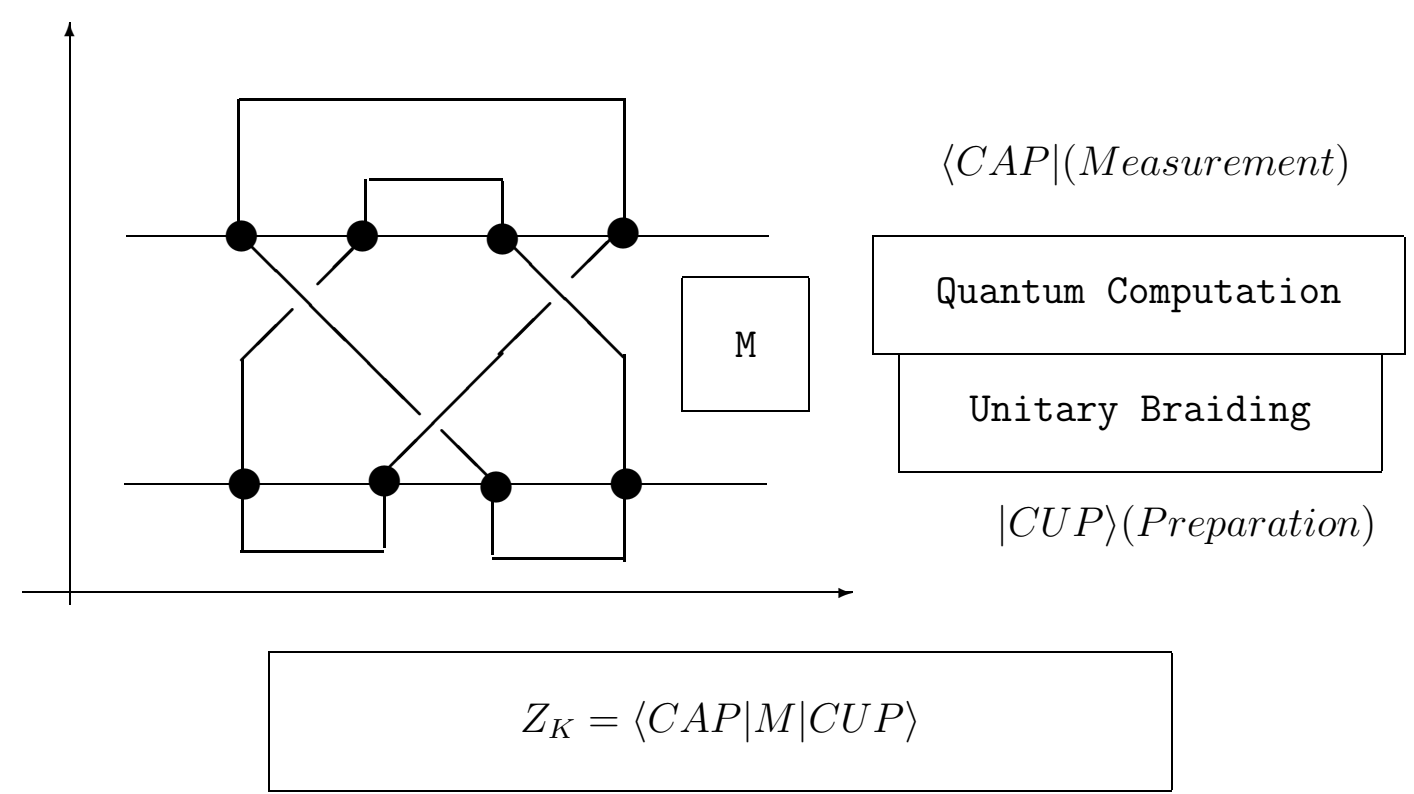

Figure 12 A Knot Quantum Computer

We should remark, however, that it is not necessary that the invariant be modeled via solutions to the Yang-Baxter equation. One can use unitary representations of the braid group that are constructed in other ways. In fact, the presently successful quantum algorithms for computing knot invariants indeed use such representations of the braid group, and we shall see this below. Nevertheless, it is useful to point out this analogy between the structure of the knot invariants and quantum computation.

Quantum algorithms for computing the Jones polynomial have been discussed elsewhere. See $[49,56,1,59,2,88]$. Here, as an example, we give a local unitary representation that can be used to compute the Jones polynomial for closures of 3-braids. We analyze this representation by making explicit how the bracket polynomial is computed from it, and showing how the quantum computation devolves to finding the trace of a unitary transformation.

The idea behind the construction of this representation depends upon the algebra generated by two single qubit density matrices (ket-bras). Let $|v\rangle$ 
and $|w\rangle$ be two qubits in $V$, a complex vector space of dimension two over the complex numbers. Let $P=|v\rangle\langle v|$ and $Q=|w\rangle\langle w|$ be the corresponding ket-bras. Note that

$$
\begin{gathered}
P^{2}=|v|^{2} P, \\
Q^{2}=|w|^{2} Q, \\
P Q P=|\langle v \mid w\rangle|^{2} P, \\
Q P Q=|\langle v \mid w\rangle|^{2} Q .
\end{gathered}
$$

$P$ and $Q$ generate a representation of the Temperley-Lieb algebra (See Section 5 of the present paper). One can adjust parameters to make a representation of the three-strand braid group in the form

$$
\begin{aligned}
& s_{1} \longmapsto r P+s I, \\
& s_{2} \longmapsto t Q+u I,
\end{aligned}
$$

where $I$ is the identity mapping on $V$ and $r, s, t, u$ are suitably chosen scalars. In the following we use this method to adjust such a representation so that it is unitary. Note also that this is a local unitary representation of $B_{3}$ to $U(2)$. We leave it as an exersise for the reader to verify that it fits into our general classification of such representations as given in section 3 of the present paper.

Here is a specific representation depending on two symmetric matrices $U_{1}$ and $U_{2}$ with

$$
U_{1}=\left[\begin{array}{ll}
d & 0 \\
0 & 0
\end{array}\right]=d|w\rangle\langle w|
$$

and

$$
U_{2}=\left[\begin{array}{cc}
d^{-1} & \sqrt{1-d^{-2}} \\
\sqrt{1-d^{-2}} & d-d^{-1}
\end{array}\right]=d|v\rangle\langle v|
$$

where $w=(1,0)$, and $v=\left(d^{-1}, \sqrt{1-d^{-2}}\right)$, assuming the entries of $v$ are real. Note that $U_{1}^{2}=d U_{1}$ and $U_{2}^{2}=d U_{1}$. Moreover, $U_{1} U_{2} U_{1}=U_{1}$ and $U_{2} U_{1} U_{2}=U_{1}$. This is an example of a specific representation of the Temperley-Lieb algebra $[37,49]$. The desired representation of the Artin braid group is given on the two braid generators for the three strand braid group by the equations:

$$
\begin{aligned}
& \Phi\left(s_{1}\right)=A I+A^{-1} U_{1}, \\
& \Phi\left(s_{2}\right)=A I+A^{-1} U_{2} .
\end{aligned}
$$

Here $I$ denotes the $2 \times 2$ identity matrix. 
For any $A$ with $d=-A^{2}-A^{-2}$ these formulas define a representation of the braid group. With $A=e^{i \theta}$, we have $d=-2 \cos (2 \theta)$. We find a specific range of angles $\theta$ in the following disjoint union of angular intervals

$$
\theta \in[0, \pi / 6] \sqcup[\pi / 3,2 \pi / 3] \sqcup[5 \pi / 6,7 \pi / 6] \sqcup[4 \pi / 3,5 \pi / 3] \sqcup[11 \pi / 6,2 \pi]
$$

that give unitary representations of the three-strand braid group. Thus a specialization of a more general represention of the braid group gives rise to a continuous family of unitary representations of the braid group.

Lemma. Note that the traces of these matrices are given by the formulas $\operatorname{tr}\left(U_{1}\right)=\operatorname{tr}\left(U_{2}\right)=d$ while $\operatorname{tr}\left(U_{1} U_{2}\right)=\operatorname{tr}\left(U_{2} U_{1}\right)=1$. If $b$ is any braid, let $I(b)$ denote the sum of the exponents in the braid word that expresses $b$. For $b$ a three-strand braid, it follows that

$$
\Phi(b)=A^{I(b)} I+\Pi(b)
$$

where $I$ is the $2 \times 2$ identity matrix and $\Pi(b)$ is a sum of products in the Temperley-Lieb algebra involving $U_{1}$ and $U_{2}$.

We omit the proof of this Lemma. It is a calculation. To see it, consider an example. Suppose that $b=s_{1} s_{2}^{-1} s_{1}$. Then

$$
\begin{aligned}
& \Phi(b)=\Phi\left(s_{1} s_{2}^{-1} s_{1}\right)=\Phi\left(s_{1}\right) \Phi\left(s_{2}^{-1}\right) \Phi\left(s_{1}\right)= \\
& \left(A I+A^{-1} U_{1}\right)\left(A^{-1} I+A U_{2}\right)\left(A I+A^{-1} U_{1}\right) .
\end{aligned}
$$

The sum of products over the generators $U_{1}$ and $U_{2}$ of the Temperley-Lieb algebra comes from expanding this expression.

Since the Temperley-Lieb algebra in this dimension is generated by $I, U_{1}$, $U_{2}, U_{1} U_{2}$ and $U_{2} U_{1}$, it follows that the value of the bracket polynomial of the closure of the braid $b$, denoted $\langle\bar{b}\rangle$, can be calculated directly from the trace of this representation, except for the part involving the identity matrix. The result is the equation

$$
<\bar{b}>=A^{I(b)} d^{2}+\operatorname{tr}(\Pi(b))
$$

where $\bar{b}$ denotes the standard braid closure of $b$, and the sharp brackets denote the bracket polynomial. From this we see at once that

$$
<\bar{b}>=\operatorname{tr}(\Phi(b))+A^{I(b)}\left(d^{2}-2\right) .
$$

It follows from this calculation that the question of computing the bracket polynomial for the closure of the three-strand braid $b$ is mathematically equivalent to the problem of computing the trace of the unitary matrix $\Phi(b)$. 


\section{The Hadamard Test}

In order to (quantum) compute the trace of a unitary matrix $U$, one can use the Hadamard test to obtain the diagonal matrix elements $\langle\psi|U| \psi\rangle$ of $U$. The trace is then the sum of these matrix elements as $|\psi\rangle$ runs over an orthonormal basis for the vector space. We first obtain

$$
\frac{1}{2}+\frac{1}{2} \operatorname{Re}\langle\psi|U| \psi\rangle
$$

as an expectation by applying the Hadamard gate $H$

$$
\begin{aligned}
& H|0\rangle=\frac{1}{\sqrt{2}}(|0\rangle+|1\rangle) \\
& H|1\rangle=\frac{1}{\sqrt{2}}(|0\rangle-|1\rangle)
\end{aligned}
$$

to the first qubit of

$$
C_{U} \circ(H \otimes 1)|0\rangle|\psi\rangle=\frac{1}{\sqrt{2}}(|0\rangle \otimes|\psi\rangle+|1\rangle \otimes U|\psi\rangle .
$$

Here $C_{U}$ denotes controlled $U$, acting as $U$ when the control bit is $|1\rangle$ and the identity mapping when the control bit is $|0\rangle$. We measure the expectation for the first qubit $|0\rangle$ of the resulting state

$$
\begin{gathered}
\frac{1}{2}(H|0\rangle \otimes|\psi\rangle+H|1\rangle \otimes U|\psi\rangle)=\frac{1}{2}((|0\rangle+|1\rangle) \otimes|\psi\rangle+(|0\rangle-|1\rangle) \otimes U|\psi\rangle) \\
=\frac{1}{2}(|0\rangle \otimes(|\psi\rangle+U|\psi\rangle)+|1\rangle \otimes(|\psi\rangle-U|\psi\rangle)) .
\end{gathered}
$$

This expectation is

$$
\frac{1}{2}\left(\left\langle\psi\left|+\langle\psi| U^{\dagger}\right)(|\psi\rangle+U|\psi\rangle)=\frac{1}{2}+\frac{1}{2} R e\langle\psi|U| \psi\rangle .\right.\right.
$$

The imaginary part is obtained by applying the same procedure to

$$
\frac{1}{\sqrt{2}}(|0\rangle \otimes|\psi\rangle-i|1\rangle \otimes U|\psi\rangle
$$

This is the method used in [1], and the reader may wish to contemplate its efficiency in the context of this simple model. Note that the Hadamard test enables this quantum computation to estimate the trace of any unitary matrix $U$ by repeated trials that estimate individual matrix entries $\langle\psi|U| \psi\rangle$. We shall return to quantum algorithms for the Jones polynomial and other knot polynomials in a subsequent paper. 


\section{Quantum Topology, Cobordism Categories, Temperley-Lieb Algebra and Topological Quan- tum Field Theory}

The purpose of this section is to discuss the general idea behind topological quantum field theory, and to illustrate its application to basic quantum mechanics and quantum mechanical formalism. It is useful in this regard to have available the concept of category, and we shall begin the section by discussing this far-reaching mathematical concept.

Definition. A category Cat consists in two related collections:

1. Obj(Cat), the objects of Cat, and

2. Morph(Cat), the morphisms of Cat.

satisfying the following axioms:

1. Each morphism $f$ is associated to two objects of Cat, the domain of $\mathrm{f}$ and the codomain of $\mathrm{f}$. Letting $A$ denote the domain of $f$ and $B$ denote the codomain of $f$, it is customary to denote the morphism $f$ by the arrow notation $f: A \longrightarrow B$.

2. Given $f: A \longrightarrow B$ and $g: B \longrightarrow C$ where $A, B$ and $C$ are objects of $C a t$, then there exists an associated morphism $g \circ f: A \longrightarrow C$ called the composition of $f$ and $g$.

3. To each object $A$ of $C a t$ there is a unique identity morphism $1_{A}: A \longrightarrow A$ such that $1_{A} \circ f=f$ for any morphism $f$ with codomain $A$, and $g \circ 1_{A}=g$ for any morphism $g$ with domain $A$.

4. Given three morphisms $f: A \longrightarrow B, g: B \longrightarrow C$ and $h: C \longrightarrow D$, then composition is associative. That is

$$
(h \circ g) \circ f=h \circ(g \circ f) .
$$

If $\mathrm{Cat}_{1}$ and $\mathrm{Cat}_{2}$ are two categories, then a functor $F: \mathrm{Cat}_{1} \longrightarrow \mathrm{Cat}_{2}$ consists in functions $\left.F_{O}: \operatorname{Obj}\left(\mathrm{Cat}_{1}\right) \longrightarrow \operatorname{Obj}_{\left(\mathrm{Cat}_{2}\right)}\right)$ and $F_{M}: \operatorname{Morph}_{\left(\mathrm{Cat}_{1}\right) \longrightarrow} \longrightarrow$ $M o r p h\left(\mathrm{Cat}_{2}\right)$ such that identity morphisms and composition of morphisms are preserved under these mappings. That is (writing just $F$ for $F_{O}$ and $F_{M}$ ),

1. $F\left(1_{A}\right)=1_{F(A)}$,

2. $F(f: A \longrightarrow B)=F(f): F(A) \longrightarrow F(B)$, 
3. $F(g \circ f)=F(g) \circ F(f)$.

A functor $F:$ Cat $_{1} \longrightarrow$ Cat $_{2}$ is a structure preserving mapping from one category to another. It is often convenient to think of the image of the functor $F$ as an interpretation of the first category in terms of the second. We shall use this terminology below and sometimes refer to an interpretation without specifying all the details of the functor that describes it.

The notion of category is a broad mathematical concept, encompassing many fields of mathematics. Thus one has the category of sets where the objects are sets (collections) and the morphisms are mappings between sets. One has the category of topological spaces where the objects are spaces and the morphisms are continuous mappings of topological spaces. One has the category of groups where the objects are groups and the morphisms are homomorphisms of groups. Functors are structure preserving mappings from one category to another. For example, the fundamental group is a functor from the category of topological spaces with base point, to the category of groups. In all the examples mentioned so far, the morphisms in the category are restrictions of mappings in the category of sets, but this is not necessarily the case. For example, any group $G$ can be regarded as a category, $\operatorname{Cat}(G)$, with one object $*$. The morphisms from $*$ to itself are the elements of the group and composition is group multiplication. In this example, the object has no internal structure and all the complexity of the category is in the morphisms.

The Artin braid group $B_{n}$ can be regarded as a category whose single object is an ordered row of points $[n]=\{1,2,3, \ldots, n\}$. The morphisms are the braids themselves and composition is the multiplication of the braids. A given ordered row of points is interpreted as the starting or ending row of points at the bottom or the top of the braid. In the case of the braid category, the morphisms have both external and internal structure. Each morphism produces a permutation of the ordered row of points (corresponding to the begiinning and ending points of the individual braid strands), and weaving of the braid is extra structure beyond the object that is its domain and codomain. Finally, for this example, we can take all the braid groups $B_{n}$ ( $n$ a positive integer) under the wing of a single category, $\operatorname{Cat}(B)$, whose objects are all ordered rows of points $[n]$, and whose morphisms are of the form $b:[n] \longrightarrow[n]$ where $b$ is a braid in $B_{n}$. The reader may wish to have morphisms between objects with different $n$. We will have this shortly in the Temperley-Lieb category and in the category of tangles.

The $n$-Cobordism Category, $\operatorname{Cob}[n]$, has as its objects smooth manifolds of dimension $n$, and as its morphisms, smooth manifolds $M^{n+1}$ of dimension $n+1$ 
with a partition of the boundary, $\partial M^{n+1}$, into two collections of $n$-manifolds that we denote by $L\left(M^{n+1}\right)$ and $R\left(M^{n+1}\right)$. We regard $M^{n+1}$ as a morphism from $L\left(M^{n+1}\right)$ to $R\left(M^{n+1}\right)$

$$
M^{n+1}: L\left(M^{n+1}\right) \longrightarrow R\left(M^{n+1}\right)
$$

As we shall see, these cobordism categories are highly significant for quantum mechanics, and the simplest one, $\operatorname{Cob}[0]$ is directly related to the Dirac notation of bras and kets and to the Temperley-Lieb algebara. We shall concentrate in this section on these cobordism categories, and their relationships with quantum mechanics.

One can choose to consider either oriented or non-oriented manifolds, and within unoriented manifolds there are those that are orientable and those that are not orientable. In this section we will implicitly discuss only orientable manifolds, but we shall not specify an orientation. In the next section, with the standard definition of topological quantum field theory, the manifolds will be oriented. The definitions of the cobordism categories for oriented manifolds go over mutatis mutandis.

Lets begin with $C o b[0]$. Zero dimensional manifolds are just collections of points. The simplest zero dimensional manifold is a single point $p$. We take $p$ to be an object of this category and also $*$, where $*$ denotes the empty manifold (i.e. the empty set in the category of manifolds). The object $*$ occurs in $\operatorname{Cob}[n]$ for every $n$, since it is possible that either the left set or the right set of a morphism is empty. A line segment $S$ with boundary points $p$ and $q$ is a morphism from $p$ to $q$.

$$
S: p \longrightarrow q
$$

See Figure 13. In this figure we have illustrated the morphism from $p$ to $p$. The simplest convention for this category is to take this morphism to be the identity. Thus if we look at the subcategory of $\operatorname{Cob}[0]$ whose only object is $p$, then the only morphism is the identity morphism. Two points occur as the boundary of an interval. The reader will note that $\operatorname{Cob}[0]$ and the usual arrow notation for morphisms are very closely related. This is a place where notation and mathematical structure share common elements. In general the objects of $C o b[0]$ consist in the empty object $*$ and non-empty rows of points, symbolized by

$$
p \otimes p \otimes \cdots \otimes p \otimes p
$$

Figure 13 also contains a morphism

$$
p \otimes p \longrightarrow *
$$


and the morphism

$$
* \longrightarrow p \otimes p \text {. }
$$

The first represents a cobordism of two points to the empty set (via the bounding curved interval). The second represents a cobordism from the empty set to two points.

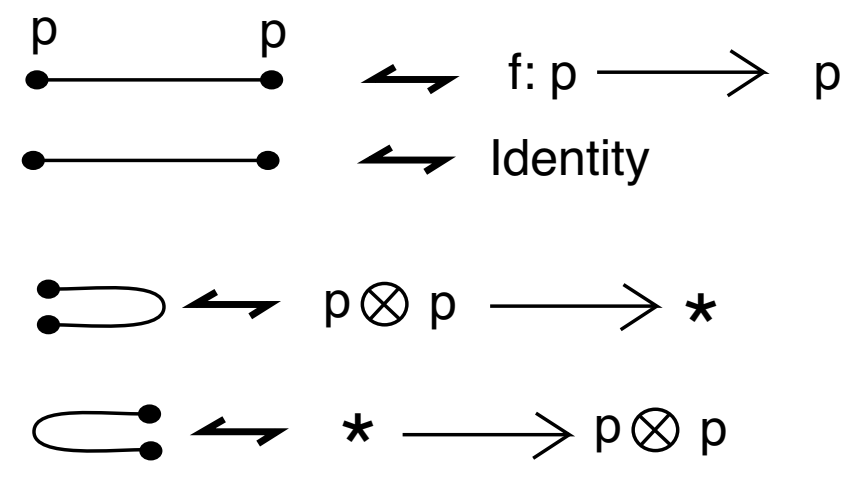

Figure 13 - Elementary Cobordisms

In Figure 14, we have indicated more morphisms in $\operatorname{Cob}[0]$, and we have named the morphisms just discussed as

$$
\begin{aligned}
& |\Omega\rangle: p \otimes p \longrightarrow *, \\
& \langle\Theta|: * \longrightarrow p \otimes p .
\end{aligned}
$$

The point to notice is that the usual conventions for handling Dirac bra-kets are essentially the same as the compostion rules in this topological category. Thus in Figure 14 we have that

$$
\langle\Theta|\circ| \Omega\rangle=\langle\Theta \mid \Omega\rangle: * \longrightarrow *
$$

represents a cobordism from the empty manifold to itself. This cobordism is topologically a circle and, in the Dirac formalism is interpreted as a scalar. In order to interpret the notion of scalar we would have to map the cobordism category to the category of vector spaces and linear mappings. We shall discuss this after describing the similarities with quantum mechanical formalism. Nevertheless, the reader should note that if $V$ is a vector space over the complex numbers $\mathcal{C}$, then a linear mapping from $\mathcal{C}$ to $\mathcal{C}$ is determined by the image of 1 , and hence is characterized by the scalar that is the image of 1 . In this sense a mapping $\mathcal{C} \longrightarrow \mathcal{C}$ can be regarded as a possible image in vector spaces of the abstract structure $\langle\Theta \mid \Omega\rangle: * \longrightarrow *$. It is therefore assumed that 
in $\operatorname{Cob}[0]$ the composition with the morphism $\langle\Theta \mid \Omega\rangle$ commutes with any other morphism. In that way $\langle\Theta \mid \Omega\rangle$ behaves like a scalar in the cobordism category. In general, an $n+1$ manifold without boundary behaves as a scalar in $\operatorname{Cob}[n]$, and if a manifold $M^{n+1}$ can be written as a union of two submanifolds $L^{n+1}$ and $R^{n+1}$ so that that an $n$-manifold $W^{n}$ is their common boundary:

$$
M^{n+1}=L^{n+1} \cup R^{n+1}
$$

with

$$
L^{n+1} \cap R^{n+1}=W^{n}
$$

then, we can write

$$
\left\langle M^{n+1}\right\rangle=\left\langle L^{n+1} \cup R^{n+1}\right\rangle=\left\langle L^{n+1} \mid R^{n+1}\right\rangle,
$$

and $\left\langle M^{n+1}\right\rangle$ will be a scalar (morphism that commutes with all other morphisms) in the category $\operatorname{Cob}[n]$.

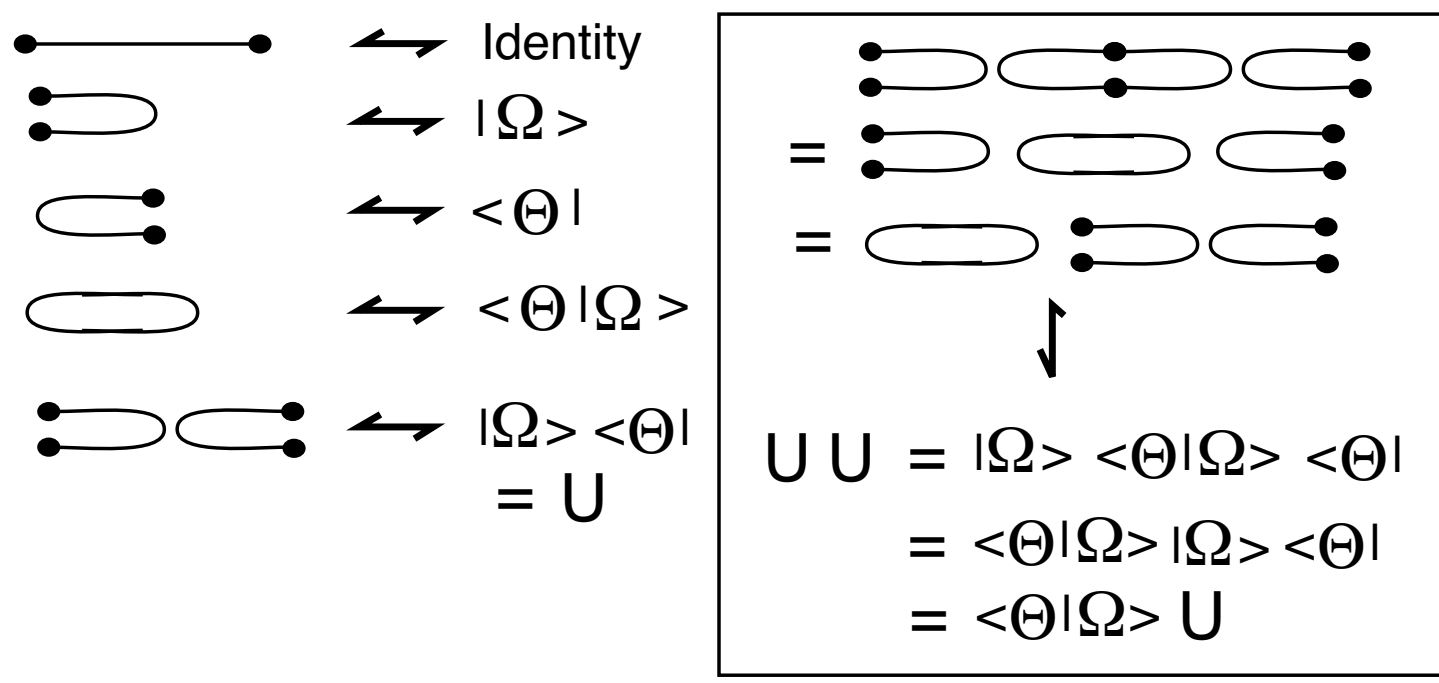

Figure 14 - Bras, Kets and Projectors 

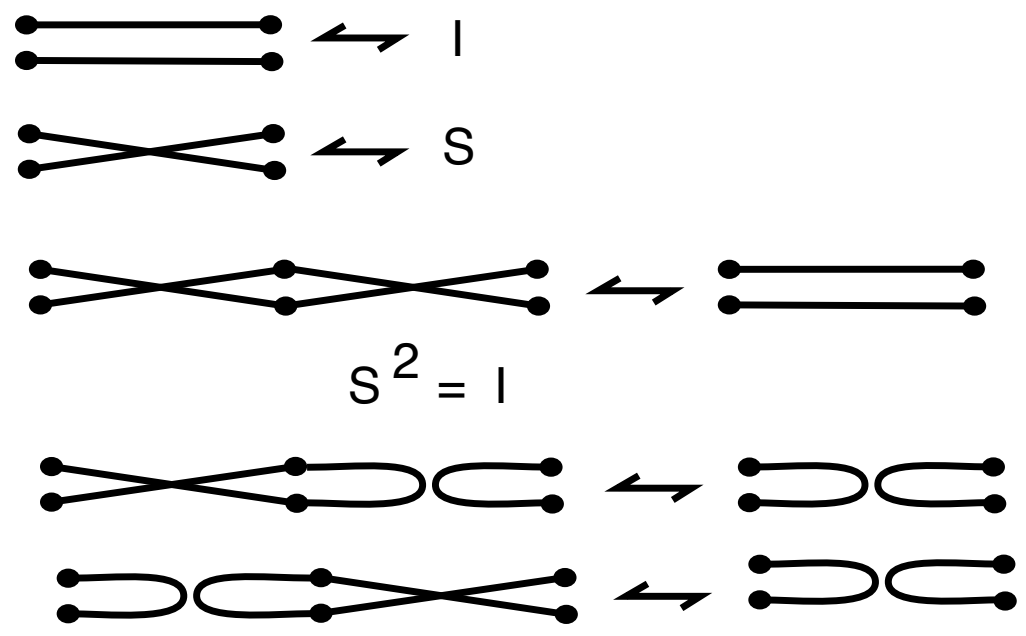

$S U=U S=U$

Figure 15 - Permutations

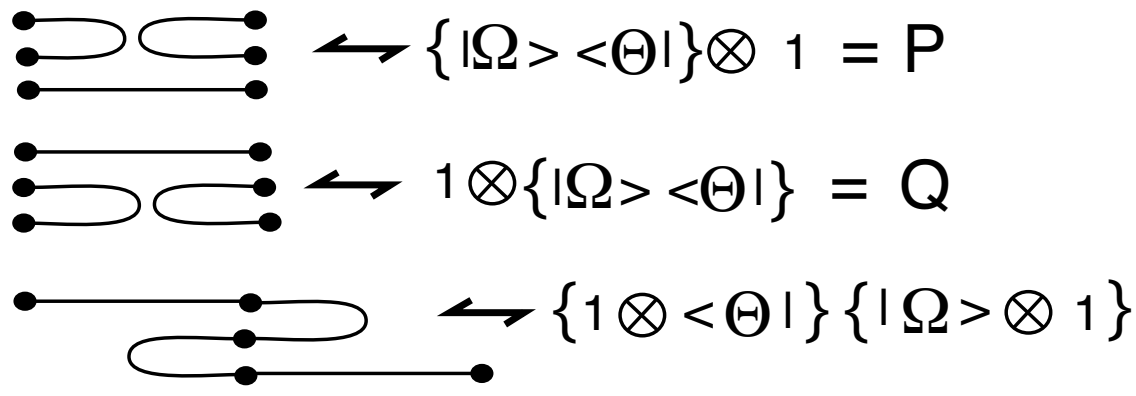

Figure 16 - Projectors in Tensor Lines and Elementary Topology

Getting back to the contents of Figure 14, note how the zero dimensional cobordism category has structural parallels to the Dirac ket-bra formalism

$$
\begin{gathered}
U=|\Omega\rangle\langle\Theta| \\
U U=|\Omega\rangle\langle\Theta \mid \Omega\rangle\langle\Theta|=\langle\Theta \mid \Omega\rangle| \Omega\rangle\langle\Theta|=\langle\Theta \mid \Omega\rangle U .
\end{gathered}
$$

In the cobordism category, the bra-ket and ket-bra formalism is seen as patterns of connection of the one-manifolds that realize the cobordisms. 
Now view Figure 15. This Figure illustrates a morphism $S$ in $\operatorname{Cob}[0]$ that requires two crossed line segments for its planar representation. Thus $S$ can be regarded as a non-trivial permutation, and $S^{2}=I$ where $I$ denotes the identity morphisms for a two-point row. From this example, it is clear that $\operatorname{Cob}[0]$ contains the structure of all the syymmetric groups and more. In fact, if we take the subcateogry of $C o b[0]$ consisting of all morphisms from $[n]$ to $[n]$ for a fixed positive integer $n$, then this gives the well-known Brauer algebra (see [13]) extending the symmetric group by allowing any connections among the points in the two rows. In this sense, one could call Cob[0] the Brauer category. We shall return to this point of view later.

In this section, we shall be concentrating on the part of $C o b[0]$ that does not involve permutations. This part can be characterized by those morphisms that can be represented by planar diagrams without crosssings between any of the line segments (the one-manifolds). We shall call this crossingless subcategory of $C o b[0]$ the Temperley-Lieb Category and denote it by CatTL. In CatTL we have the subcategory $T L[n]$ whose only objects are the row of $n$ points and the empty object $*$, and whose morphisms can all be represented by configurations that embed in the plane as in the morphisms $P$ and $Q$ in Figure 16. Note that with the empty object $*$, the morphism whose diagram is a single loop appears in $T L[n]$ and is taken to commute with all other morphisms.

The Temperley-Lieb Algebra, AlgTL[n] is generated by the morphisms in $T L[n]$ that go from $[n]$ to itself. Up to multiplication by the loop, the product (composition) of two such morphisms is another flat morphism from $[n]$ to itself. For algebraic purposes the loop $* \longrightarrow *$ is taken to be a scalar algebraic variable $\delta$ that commutes with all elements in the algebra. Thus the equation

$$
U U=\langle\Theta \mid \Omega\rangle U
$$

becomes

$$
U U=\delta U
$$

in the algebra. In the algebra we are allowed to add morphisms formally and this addition is taken to be commutative. Initially the algebra is taken with coefficients in the integers, but a different commutative ring of coefficients can be chosen and the value of the loop may be taken in this ring. For example, for quantum mechanical applications it is natural to work over the complex numbers. The multiplicative structure of $A l g T L[n]$ can be described by generators and relations as follows: Let $I_{n}$ denote the identity morphism from $[n]$ to $[n]$. Let $U_{i}$ denote the morphism from $[n]$ to $[n]$ that connects $k$ with $k$ for $k<i$ and $k>i+1$ from one row to the other, and connects $i$ to $i+1$ in each 
row. Then the algebra $\operatorname{Alg} T L[n]$ is generated by $\left\{I_{n}, U_{1}, U_{2}, \cdots, U_{n-1}\right\}$ with relations

$$
\begin{gathered}
U_{i}^{2}=\delta U_{i} \\
U_{i} U_{i+1} U_{i}=U_{i} \\
U_{i} U_{j}=U_{j} U_{i}:|i-j|>1 .
\end{gathered}
$$

These relations are illustrated for three strands in Figure 16. We leave the commuting relation for the reader to draw in the case where $n$ is four or greater. For a proof that these are indeed all the relations, see [52].

Figures 16 and 17 indicate how the zero dimensional cobordism category contains structure that goes well beyond the usual Dirac formalism. By tensoring the ket-bra on one side or another by identity morphisms, we obtain the beginnings of the Temperley-Lieb algebra and the Temperley-Lieb category. Thus Figure 17 illustrates the morphisms $P$ and $Q$ obtained by such tensoring, and the relation $P Q P=P$ which is the same as $U_{1} U_{2} U_{1}=U_{1}$

Note the composition at the bottom of the Figure 17. Here we see a composition of the identity tensored with a ket, followed by a bra tensored with the identity. The diagrammatic for this association involves "straightening" the curved structure of the morphism to a straight line. In Figure 18 we have elaborated this situation even further, pointing out that in this category each of the morphisms $\langle\Theta|$ and $|\Omega\rangle$ can be seen, by straightening, as mappings from the generating object to itself. We have denoted these corresponding morphisms by $\Theta$ and $\Omega$ respectively. In this way there is a correspondence between morphisms $p \otimes p \longrightarrow *$ and morphims $p \longrightarrow p$.

In Figure 18 we have illustrated the generalization of the straightening procedure of Figure 17. In Figure 17 the straightening occurs because the connection structure in the morphism of $C o b[0]$ does not depend on the wandering of curves in diagrams for the morphisms in that category. Nevertheless, one can envisage a more complex interpretation of the morphisms where each one-manifold (line segment) has a label, and a multiplicity of morphisms can correspond to a single line segment. This is exactly what we expect in interpretations. For example, we can interpret the line segment [1] $\longrightarrow[1]$ as a mapping from a vector space $V$ to itself. Then [1] $\longrightarrow[1]$ is the diagrammatic abstraction for $V \longrightarrow V$, and there are many instances of linear mappings from $V$ to $V$.

At the vector space level there is a duality between mappings $V \otimes V \longrightarrow \mathcal{C}$ and linear maps $V \longrightarrow V$. Specifically, let

$$
\{|0\rangle, \cdots,|m\rangle\}
$$


be a basis for $V$. Then $\Theta: V \longrightarrow V$ is determined by

$$
\Theta|i\rangle=\Theta_{i j}|j\rangle
$$

(where we have used the Einstein summation convention on the repeated index j) corresponds to the bra

$$
\langle\Theta|: V \otimes V \longrightarrow \mathcal{C}
$$

defined by

$$
\langle\Theta \mid i j\rangle=\Theta_{i j}
$$

Given $\langle\Theta|: V \otimes V \longrightarrow \mathcal{C}$, we associate $\Theta: V \longrightarrow V$ in this way.

Comparing with the diagrammatic for the category $\operatorname{Cob}[0]$, we say that $\Theta: V \longrightarrow V$ is obtained by straightening the mapping

$$
\langle\Theta|: V \otimes V \longrightarrow \mathcal{C} \text {. }
$$

Note that in this interpretation, the bras and kets are defined relative to the tensor product of $V$ with itself and [2] is interpreted as $V \otimes V$. If we interpret [2] as a single vector space $W$, then the usual formalisms of bras and kets still pass over from the cobordism category.

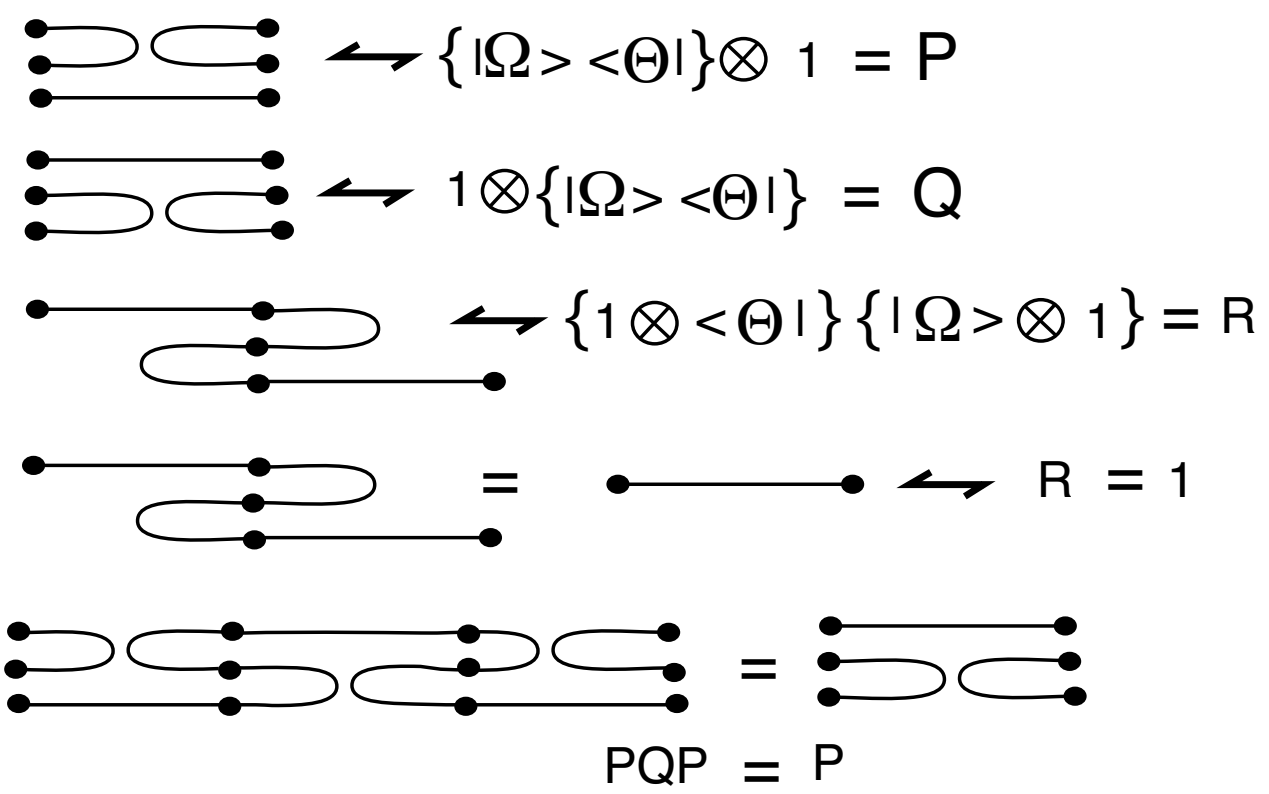

Figure 17 - The Basic Temperley-Lieb Relation 

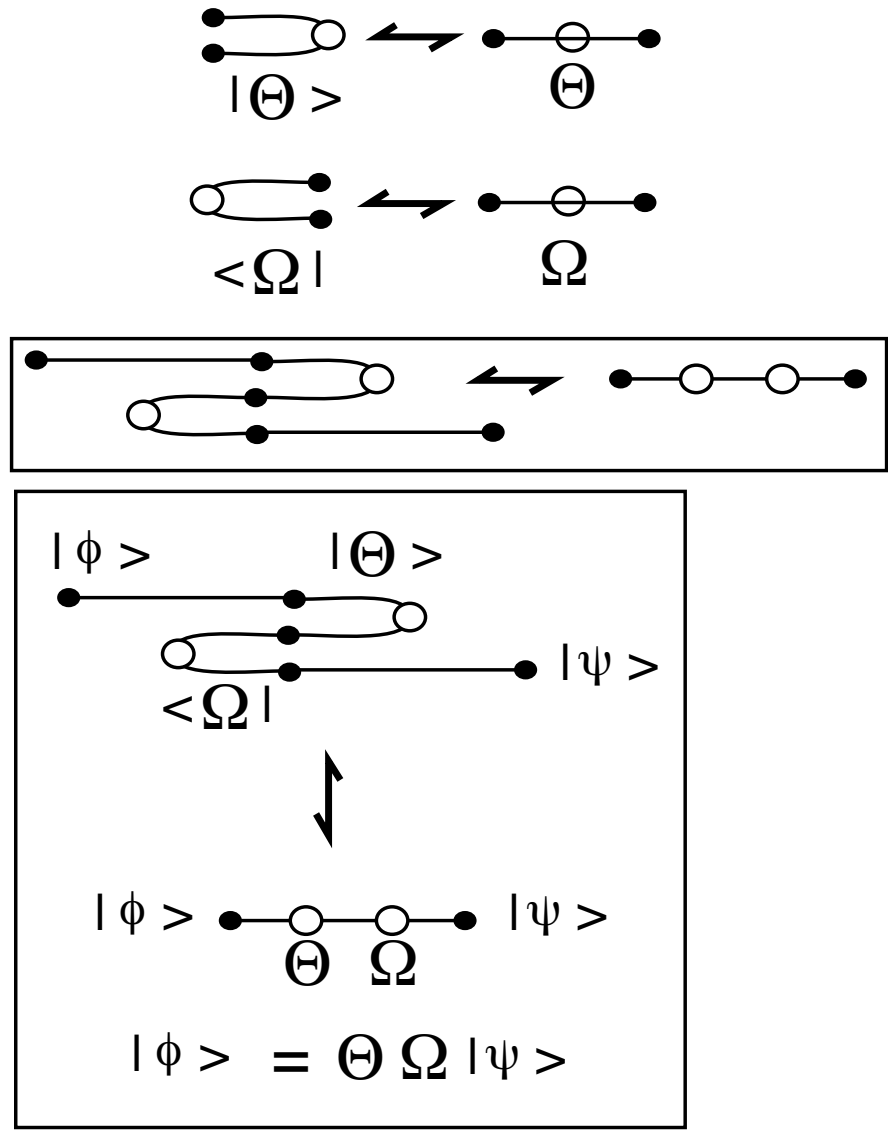

Figure 18 - The Key to Teleportation

Figure 18 illustrates the staightening of $|\Theta\rangle$ and $\langle\Omega|$, and the straightening of a composition of these applied to $|\psi\rangle$, resulting in $|\phi\rangle$. In the left-hand part of the bottom of Figure 18 we illustrate the preparation of the tensor product $|\Theta\rangle \otimes|\psi\rangle$ followed by a successful measurement by $\langle\Omega|$ in the second two tensor factors. The resulting single qubit state, as seen by straightening, is $|\phi\rangle=\Theta \circ \Omega|\psi\rangle$.

From this, we see that it is possible to reversibly, indeed unitarily, transform a state $|\psi\rangle$ via a combination of preparation and measurement just so long as the straightenings of the preparation and measurement $(\Theta$ and $\Omega$ ) are each invertible (unitary). This is the key to teleportation $[51,20,21]$. In the standard teleportation procedure one chooses the preparation $\Theta$ to be (up to normalization) the 2 dimensional identity matrix so that $|\theta\rangle=|00\rangle+|11\rangle$. If the successful measurement $\Omega$ is also the identity, then the transmitted state $|\phi\rangle$ will be equal to $|\psi\rangle$. In general we will have $|\phi\rangle=\Omega|\psi\rangle$. One can then choose a basis of measurements $|\Omega\rangle$, each corresponding to a unitary transformation $\Omega$ 
so that the recipient of the transmission can rotate the result by the inverse of $\Omega$ to reconsitute $|\psi\rangle$ if he is given the requisite information. This is the basic design of the teleportation procedure.

There is much more to say about the category $\operatorname{Cob}[0]$ and its relationship with quantum mechanics. We will stop here, and invite the reader to explore further. Later in this paper, we shall use these ideas in formulating our representations of the braid group. For now, we point out how things look as we move upward to $\operatorname{Cob}[n]$ for $n>0$. In Figure 19 we show typical cobordisms (morphisms) in $\operatorname{Cob}[1]$ from two circles to one circle and from one circle to two circles. These are often called "pairs of pants". Their composition is a surface of genus one seen as a morphism from two circles to two circles. The bottom of the figure indicates a ket-bra in this dimension in the form of a mapping from one circle to one circle as a composition of a cobordism of a circle to the empty set and a cobordism from the empty set to a circle (circles bounding disks). As we go to higher dimensions the structure of cobordisms becomes more interesting and more complicated. It is remarkable that there is so much structure in the lowest dimensions of these categories.

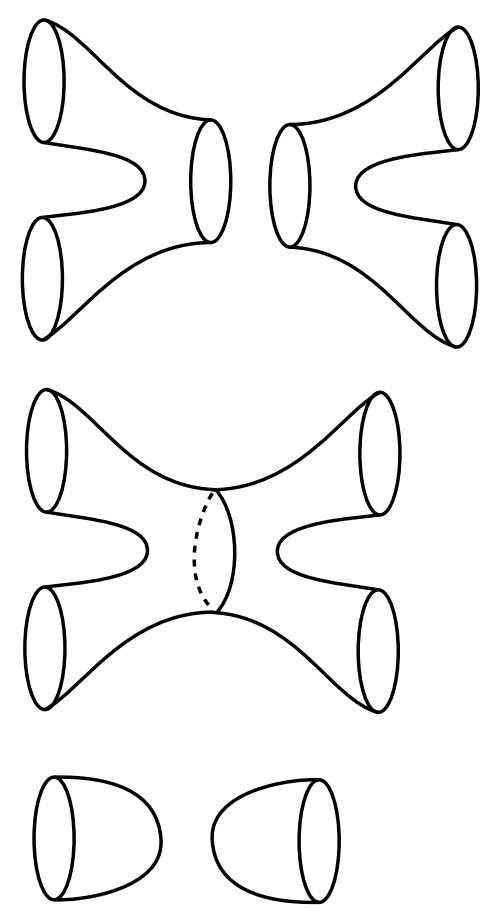

Figure 19 - Corbordisms of 1-Manifolds are Surfaces 


\section{Braiding and Topological Quantum Field The- ory}

The purpose of this section is to discuss in a very general way how braiding is related to topological quantum field theory. In the section to follow, we will use the Temperley-Lieb recoupling theory to produce specfic unitary representations of the Artin braid group.

The ideas in the subject of topological quantum field theory (TQFT) are well expressed in the book [6] by Michael Atiyah and the paper [87] by Edward Witten. Here is Atiyah's definition:

Definition. A TQFT in dimension $d$ is a functor $Z(\Sigma)$ from the cobordism category $C o b[d]$ to the category $V e c t$ of vector spaces and linear mappings which assigns

1. a finite dimensional vector space $Z(\Sigma)$ to each compact, oriented $d$ dimensional manifold $\Sigma$,

2. a vector $Z(Y) \in Z(\Sigma)$ for each compact, oriented $(d+1)$-dimensional manifold $Y$ with boundary $\Sigma$.

3. a linear mapping $Z(Y): Z\left(\Sigma_{1}\right) \longrightarrow Z\left(\Sigma_{2}\right)$ when $Y$ is a $(d+1)$-manifold that is a cobordism between $\Sigma_{1}$ and $\Sigma_{2}$ (whence the boundary of $Y$ is the union of $\Sigma_{1}$ and $-\Sigma_{2}$.

The functor satisfies the following axioms.

1. $Z\left(\Sigma^{\dagger}\right)=Z(\Sigma)^{\dagger}$ where $\Sigma^{\dagger}$ denotes the manifold $\Sigma$ with the opposite orientation and $Z(\Sigma)^{\dagger}$ is the dual vector space.

2. $Z\left(\Sigma_{1} \cup \Sigma_{2}\right)=Z\left(\Sigma_{1}\right) \otimes Z\left(\Sigma_{2}\right)$ where $\cup$ denotes disjoint union.

3. If $Y_{1}$ is a cobordism from $\Sigma_{1}$ to $\Sigma_{2}, Y_{2}$ is a cobordism from $\Sigma_{2}$ to $\Sigma_{3}$ and $Y$ is the composite cobordism $Y=Y_{1} \cup_{\Sigma_{2}} Y_{2}$, then

$$
Z(Y)=Z\left(Y_{2}\right) \circ Z\left(Y_{1}\right): Z\left(\Sigma_{1}\right) \longrightarrow Z\left(\Sigma_{2}\right)
$$

is the composite of the corresponding linear mappings.

4. $Z(\phi)=\mathcal{C}(\mathcal{C}$ denotes the complex numbers $)$ for the empty manifold $\phi$.

5. With $\Sigma \times I$ (where $I$ denotes the unit interval) denoting the identity cobordism from $\Sigma$ to $\Sigma, Z(\Sigma \times I)$ is the identity mapping on $Z(\Sigma)$. 
Note that, in this view a TQFT is basically a functor from the cobordism categories defined in the last section to Vector Spaces over the complex numbers. We have already seen that in the lowest dimensional case of cobordisms of zero-dimensional manifolds, this gives rise to a rich structure related to quatum mechanics and quantum information theory. The remarkable fact is that the case of three-dimensions is also related to quantum theory, and to the lower-dimensional versions of the TQFT. This gives a significant way to think about three-manifold invariants in terms of lower dimensional patterns of interaction. Here follows a brief description.

Regard the three-manifold as a union of two handlebodies with boundary an orientable surface $S_{g}$ of genus $g$. The surface is divided up into trinions as illustrated in Figure 20. A trinion is a surface with boundary that is topologically equivalent to a sphere with three punctures. The trinion constitutes, in itself a cobordism in $C o b[1]$ from two circles to a single circle, or from a single circle to two circles, or from three circles to the empty set. The pattern of a trinion is a trivalent graphical vertex, as illustrated in Figure 20. In that figure we show the trivalent vertex graphical pattern drawn on the surface of the trinion, forming a graphical pattern for this combordism. It should be clear from this figure that any cobordism in $\operatorname{Cob}[1]$ can be diagrammed by a trivalent graph, so that the category of trivalent graphs (as morphisms from ordered sets of points to ordered sets of points) has an image in the category of cobordisms of compact one-dimensional manifolds. Given a surface $S$ (possibly with boundary) and a decomposition of that surface into triions, we associate to it a trivalent graph $G(S, t)$ where $t$ denotes the particular trinion decomposition.

In this correspondence, distinct graphs can correspond to topologically identical cobordisms of circles, as illustrated in Figure 22. It turns out that the graphical structure is important, and that it is extraordinarily useful to articulate transformations between the graphs that correspond to the homeomorphisms of the corresponding surfaces. The beginning of this structure is indicated in the bottom part of Figure 22.

In Figure 23 we illustrate another feature of the relationship betweem surfaces and graphs. At the top of the figure we indicate a homeomorphism between a twisted trinion and a standard trinion. The homeomorphism leaves the ends of the trinion (denoted $A, B$ and $C$ ) fixed while undoing the internal twist. This can be accomplished as an ambient isotopy of the embeddings in three dimensional space that are indicated by this figure. Below this isotopy we indicate the corresponding graphs. In the graph category there will have 
to be a transformation between a braided and an unbraided trivalent vertex that corresponds to this homeomorphism.
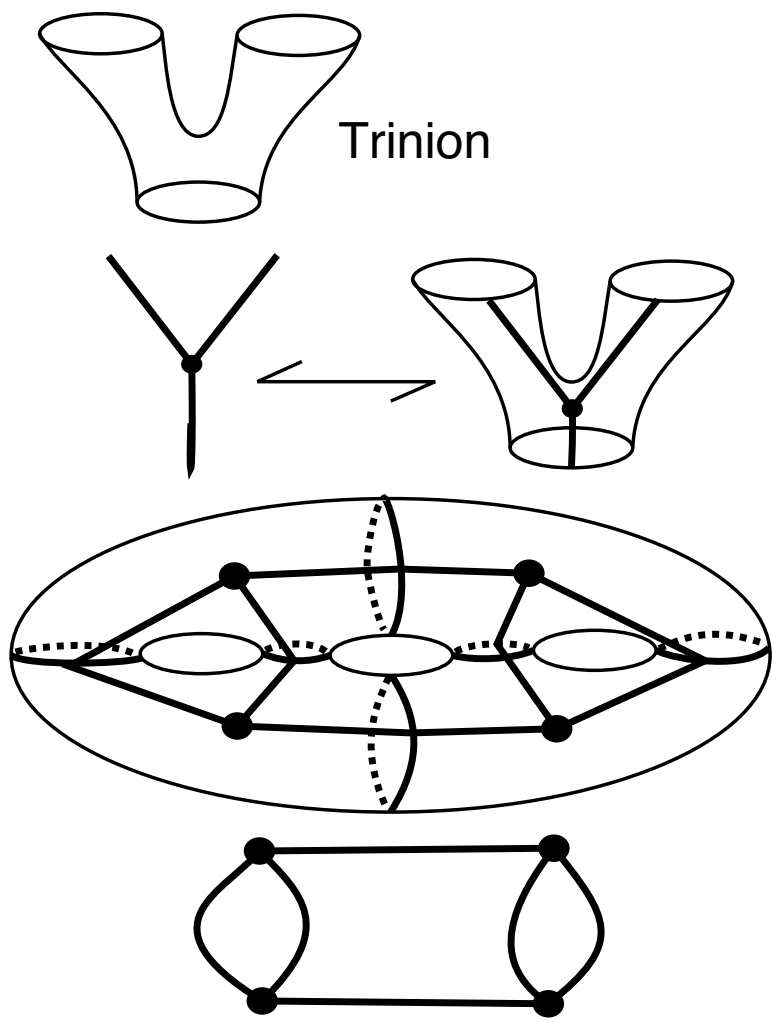

Figure 20 - Decomposition of a Surface into Trinions
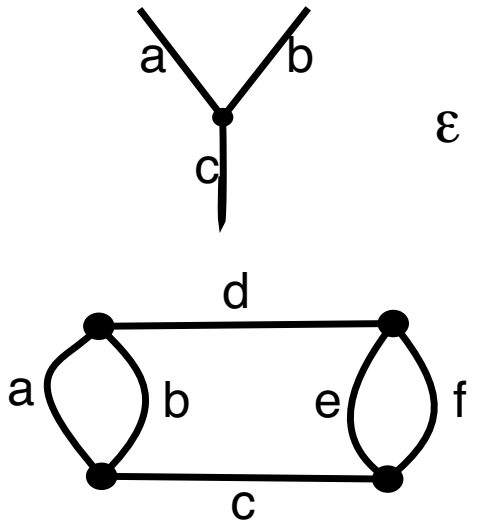
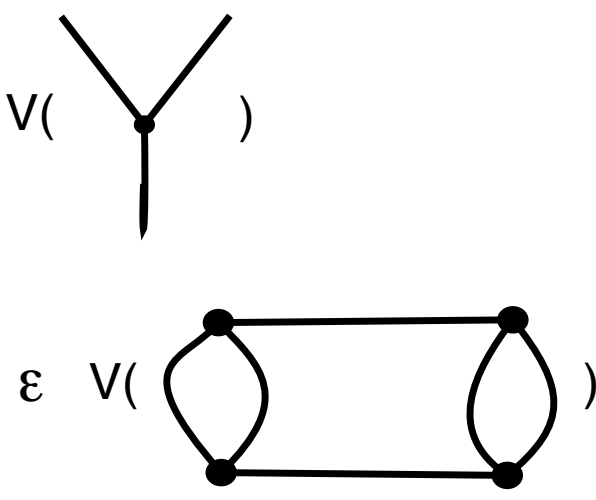

Figure 21 - Trivalent Vectors 

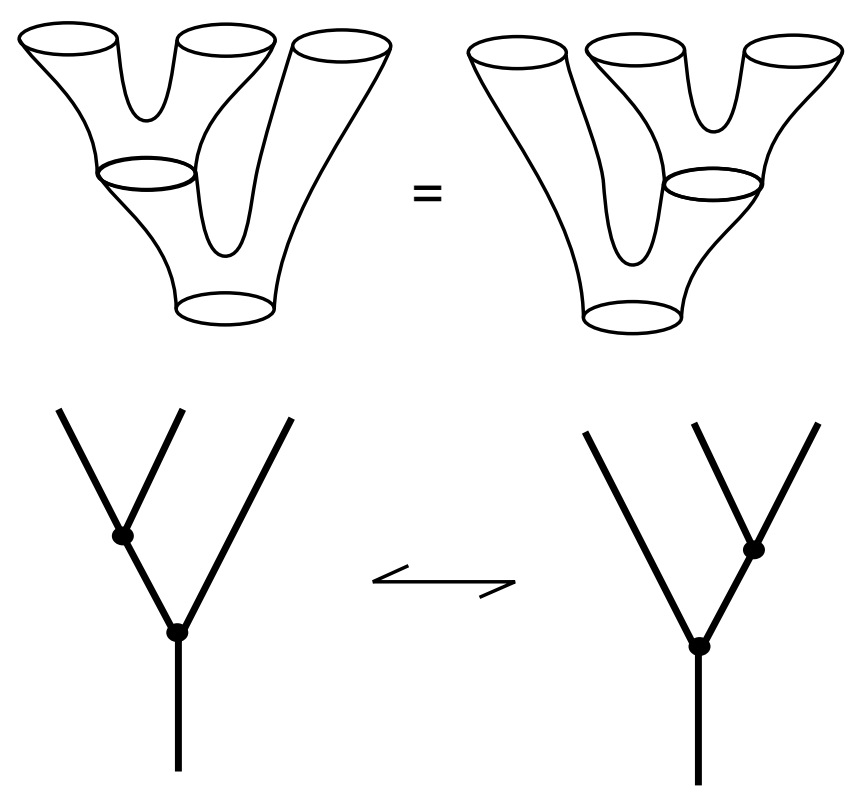

Figure 22 - Trinion Associativity
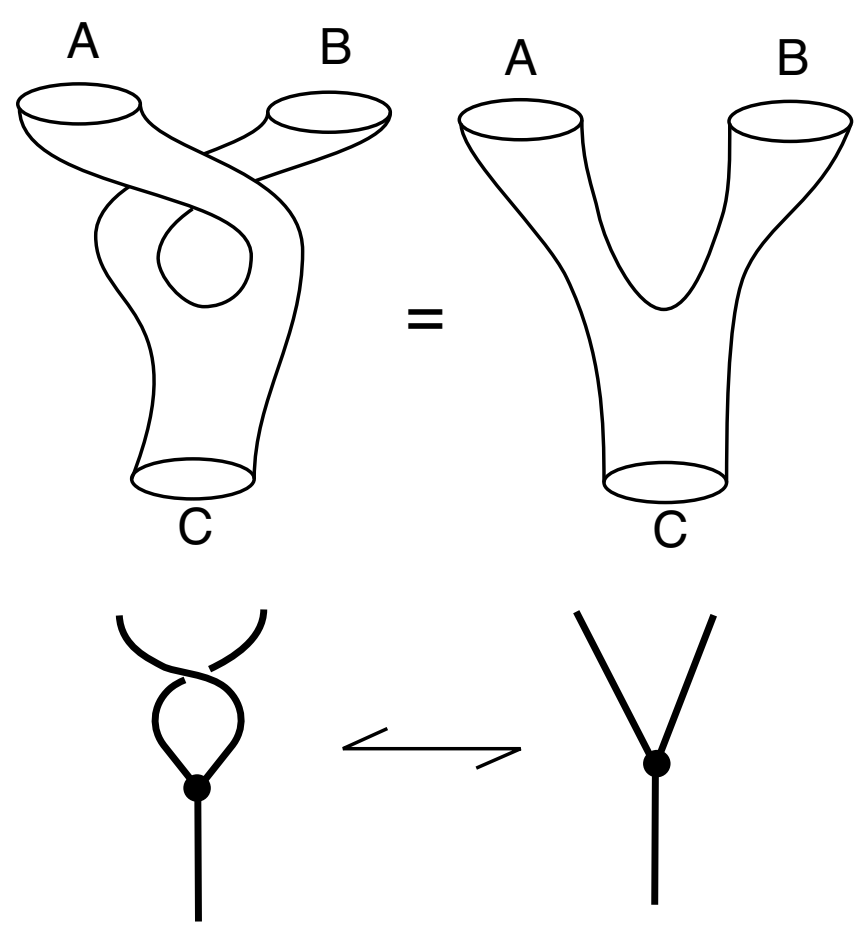

Figure 23 - Tube Twist 
From the point of view that we shall take in this paper, the key to the mathematical structure of three-dimensional TQFT lies in the trivalent graphs, including the braiding of grapical arcs. We can think of these braided graphs as representing idealized Feynman diagrams, with the trivalent vertex as the basic particle interaction vertex, and the braiding of lines representing an interaction resulting from an exchange of particles. In this view one thinks of the particles as moving in a two-dimensional medium, and the diagrams of braiding and trivalent vertex interactions as indications of the temporal events in the system, with time indicated in the direction of the morphisms in the category. Adding such graphs to the category of knots and links is an extension of the tangle category where one has already extended braids to allow any embedding of strands and circles that start in $n$ ordered points and end in $m$ ordered points. The tangle category includes the braid category and the Temperley-Lieb category. These are both included in the category of braided trivalent graphs.

Thinking of the basic trivalent vertex as the form of a particle interaction there will be a set of particle states that can label each arc incident to the vertex. In Figure 21 we illustrate the labeling of the trivalent graphs by such particle states. In the next two sections we will see specific rules for labeling such states. Here it suffices to note that there will be some restrictions on these labels, so that a trivalent vertex has a set of possible labelings. Similarly, any trivalent graph will have a set of admissible labelings. These are the possible particle processes that this graph can support. We take the set of admissible labelings of a given graph $G$ as a basis for a vector space $V(G)$ over the complex numbers. This vector space is the space of processes associated with the graph $G$. Given a surface $S$ and a decomposition $t$ of the surface into trinions, we have the associated graph $G(S, t)$ and hence a vector space of processes $V(G(S, t))$. It is desirable to have this vector space independent of the particular decomposition into trinions. If this can be accomplished, then the set of vector spaces and linear mappings associated to the surfaces can consitute a functor from the category of cobordisms of one-manifolds to vector spaces, and hence gives rise to a one-dimensional topological quantum field theory. To this end we need some properties of the particle interactions that will be described below.

A spin network is, by definition a lableled trivalent graph in a category of graphs that satisfy the properties outlined in the previous paragraph. We shall detail the requirements below.

The simplest case of this idea is C. N. Yang's original interpretation of the Yang-Baxter equation [89]. Yang articulated a quantum field theory in 
one dimension of space and one dimension of time in which the $R$-matrix giving the scattering ampitudes for an interaction of two particles whose (let us say) spins corresponded to the matrix indices so that $R_{a b}^{c d}$ is the amplitude for particles of spin $a$ and spin $b$ to interact and produce particles of spin $c$ and $d$. Since these interactions are between particles in a line, one takes the convention that the particle with spin $a$ is to the left of the particle with spin $b$, and the particle with spin $c$ is to the left of the particle with spin $d$. If one follows the concatenation of such interactions, then there is an underlying permutation that is obtained by following strands from the bottom to the top of the diagram (thinking of time as moving up the page). Yang designed the Yang-Baxter equation for $R$ so that the amplitudes for a composite process depend only on the underlying permutation corresponding to the process and not on the individual sequences of interactions.

In taking over the Yang-Baxter equation for topological purposes, we can use the same interpretation, but think of the diagrams with their under- and over-crossings as modeling events in a spacetime with two dimensions of space and one dimension of time. The extra spatial dimension is taken in displacing the woven strands perpendicular to the page, and allows us to use braiding operators $R$ and $R^{-1}$ as scattering matrices. Taking this picture to heart, one can add other particle properties to the idealized theory. In particular one can add fusion and creation vertices where in fusion two particles interact to become a single particle and in creation one particle changes (decays) into two particles. These are the trivalent vertices discussed above. Matrix elements corresponding to trivalent vertices can represent these interactions. See Figure 24.

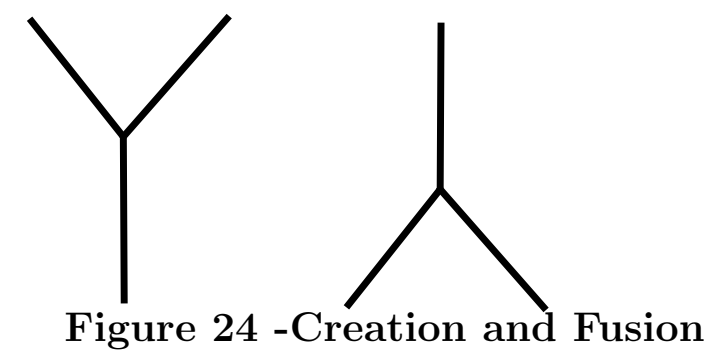

Once one introduces trivalent vertices for fusion and creation, there is the question how these interactions will behave in respect to the braiding operators. There will be a matrix expression for the compositions of braiding and fusion or creation as indicated in Figure 25. Here we will restrict ourselves to showing the diagrammatics with the intent of giving the reader a flavor of these structures. It is natural to assume that braiding intertwines with creation as shown in 
Figure 27 (similarly with fusion). This intertwining identity is clearly the sort of thing that a topologist will love, since it indicates that the diagrams can be interpreted as embeddings of graphs in three-dimensional space, and it fits with our interpretation of the vertices in terms of trinions. Figure 25 illustrates the Yang-Baxter equation. The intertwining identity is an assumption like the Yang-Baxter equation itself, that simplifies the mathematical structure of the model.

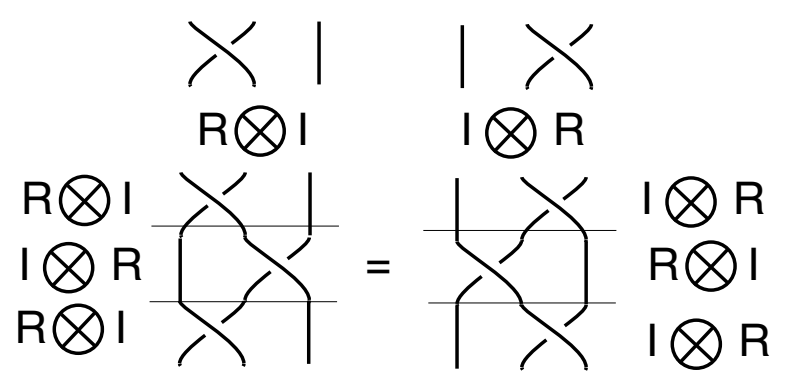

Figure 25 - YangBaxterEquation

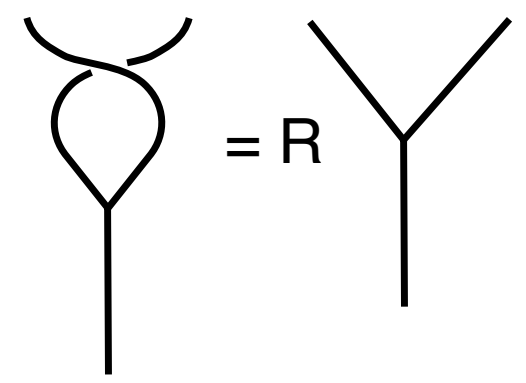

Figure 26 - Braiding

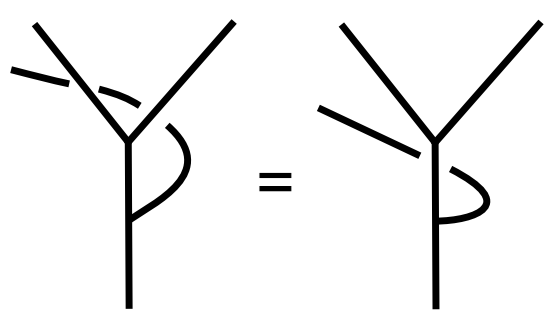

Figure 27 - Intertwining 
It is to be expected that there will be an operator that expresses the recoupling of vertex interactions as shown in Figure 28 and labeled by $Q$. This corresponds to the associativity at the level of trinion combinations shown in Figure 22. The actual formalism of such an operator will parallel the mathematics of recoupling for angular momentum. See for example [39]. If one just considers the abstract structure of recoupling then one sees that for trees with four branches (each with a single root) there is a cycle of length five as shown in Figure 29. One can start with any pattern of three vertex interactions and go through a sequence of five recouplings that bring one back to the same tree from which one started. It is a natural simplifying axiom to assume that this composition is the identity mapping. This axiom is called the pentagon identity.

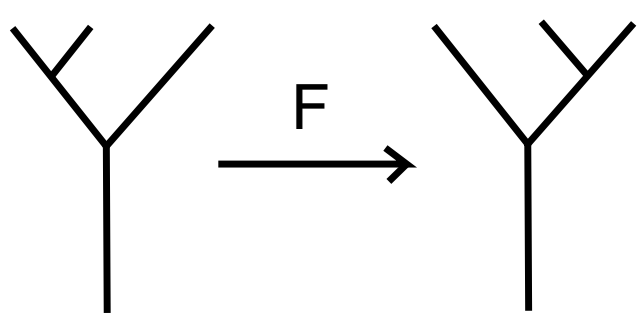

Figure 28 - Recoupling

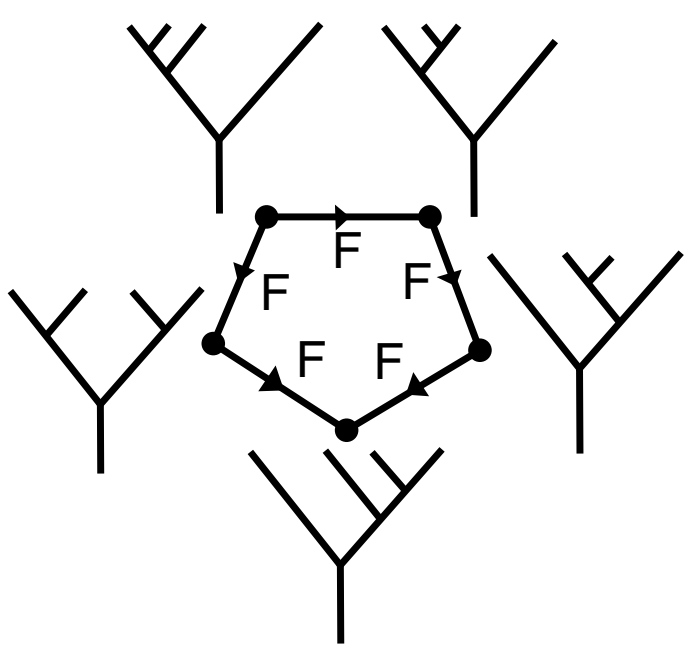

Figure 29 - Pentagon Identity 
Finally there is a hexagonal cycle of interactions between braiding, recoupling and the intertwining identity as shown in Figure 30. One says that the interactions satisfy the hexagon identity if this composition is the identity.

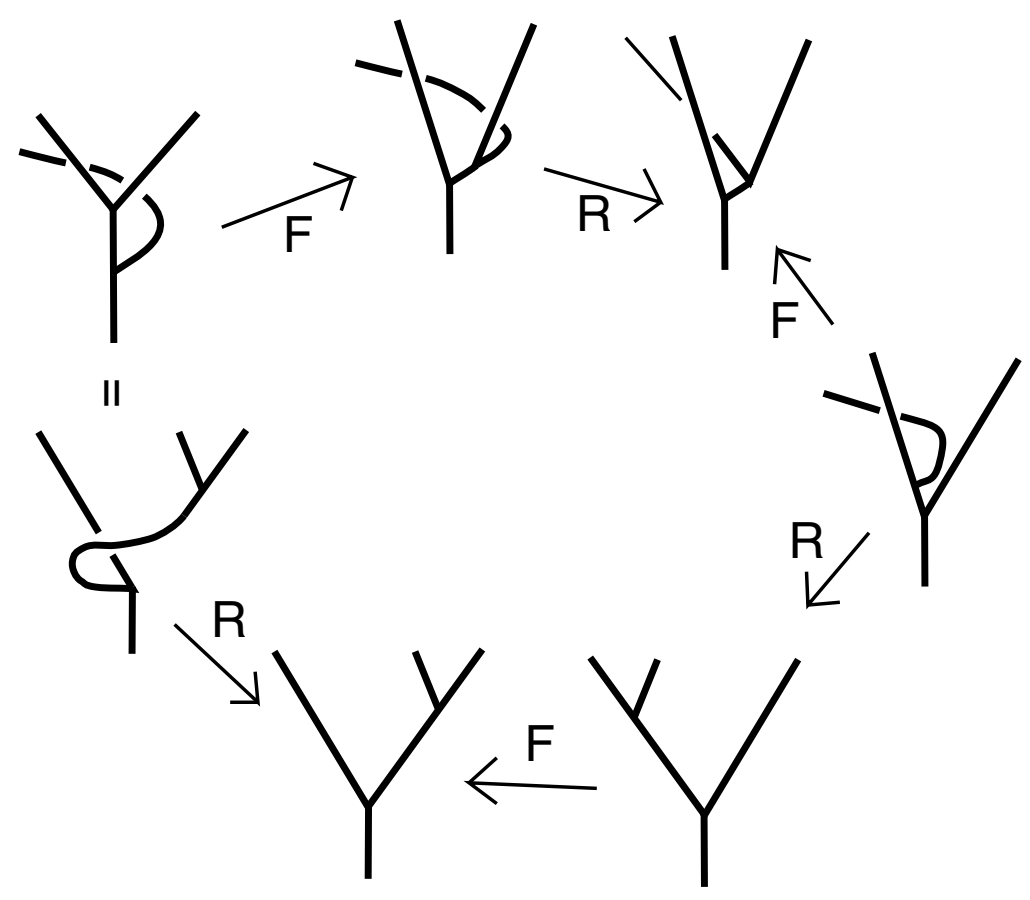

Figure 30 - Hexagon Identity

A graphical three-dimensional topological quantum field theory is an algebra of interactions that satisfies the Yang-Baxter equation, the intertwining identity, the pentagon identity and the hexagon identity. There is not room in this summary to detail the way that these properties fit into the topology of knots and three-dimensional manifolds, but a sketch is in order. For the case of topological quantum field theory related to the group $S U(2)$ there is a construction based entirely on the combinatorial topology of the bracket polynomial (See Sections 7,9 and 10 of this article.). See [44, 39] for more information on this approach.

Now return to Figure 20 where we illustrate trinions, shown in relation to a trivalent vertex, and a surface of genus three that is decomposed into four trinions. It turns out that the vector space $V\left(S_{g}\right)=V\left(G\left(S_{g}, t\right)\right)$ to a surface with a trinion decomposition as $t$ described above, and defined in 
terms of the graphical topological quantum field theory, does not depend upon the choice of trinion decomposition. This independence is guaranteed by the braiding, hexagon and pentagon identities. One can then associate a welldefined vector $|M\rangle$ in $V\left(S_{g}\right)$ whenenver $M$ is a three manifold whose boundary is $S_{g}$. Furthermore, if a closed three-manifold $M^{3}$ is decomposed along a surface $S_{g}$ into the union of $M_{-}$and $M_{+}$where these parts are otherwise disjoint threemanifolds with boundary $S_{g}$, then the inner product $I(M)=\left\langle M_{-} \mid M_{+}\right\rangle$is, up to normalization, an invariant of the three-manifold $M_{3}$. With the definition of graphical topological quantum field theory given above, knots and links can be incorporated as well, so that one obtains a source of invariants $I\left(M^{3}, K\right)$ of knots and links in orientable three-manifolds. Here we see the uses of the relationships that occur in the higher dimensional cobordism categories, as descirbed in the previous section.

The invariant $I\left(M^{3}, K\right)$ can be formally compared with the Witten [87] integral

$$
Z\left(M^{3}, K\right)=\int D A e^{(i k / 4 \pi) S(M, A)} W_{K}(A)
$$

It can be shown that up to limits of the heuristics, $Z(M, K)$ and $I\left(M^{3}, K\right)$ are essentially equivalent for appropriate choice of gauge group and corresponding spin networks.

By these graphical reformulations, a three-dimensional TQFT is, at base, a highly simplified theory of point particle interactions in $2+1$ dimensional spacetime. It can be used to articulate invariants of knots and links and invariants of three manifolds. The reader interested in the $S U(2)$ case of this structure and its implications for invariants of knots and three manifolds can consult $[39,44,65,19,70]$. One expects that physical situations involving $2+1$ spacetime will be approximated by such an idealized theory. There are also applications to $3+1$ quantum gravity $[7,8,53]$. Aspects of the quantum Hall effect may be related to topological quantum field theory [86]. One can study a physics in two dimensional space where the braiding of particles or collective excitations leads to non-trival representations of the Artin braid group. Such particles are called Anyons. Such TQFT models would describe applicable physics. One can think about applications of anyons to quantum computing along the lines of the topoological models described here. 

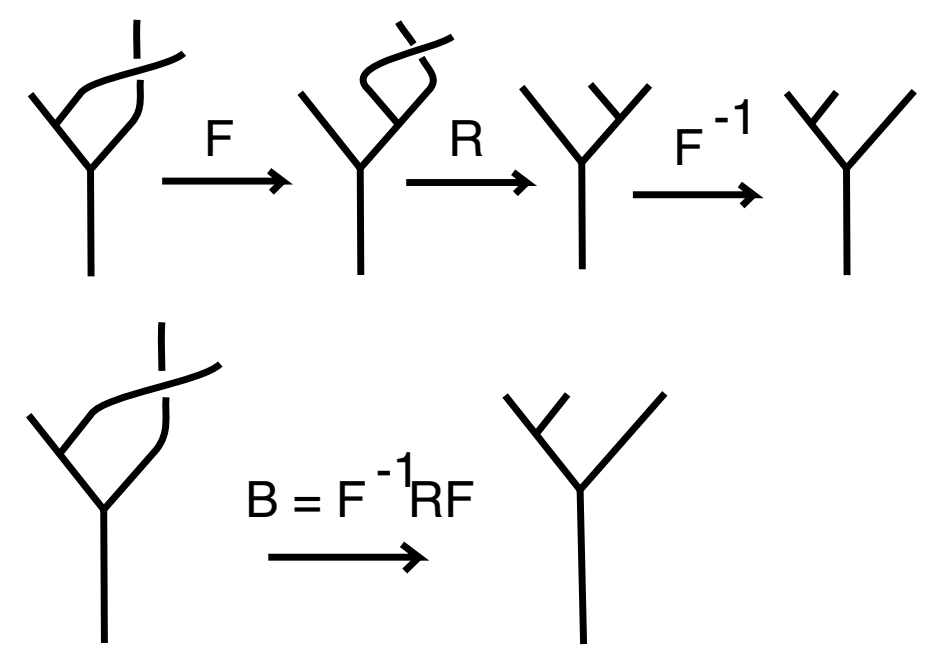

\section{Figure 31 - A More Complex Braiding Operator}

A key point in the application of TQFT to quantum information theory is contained in the structure illustrated in Figure 31. There we show a more complex braiding operator, based on the composition of recoupling with the elementary braiding at a vertex. (This structure is implicit in the Hexagon identity of Figure 30.) The new braiding operator is a source of unitary representations of braid group in situations (which exist mathematically) where the recoupling transformations are themselves unitary. This kind of pattern is utilized in the work of Freedman and collaborators [27, 28, 29, 30, 31] and in the case of classical angular momentum formalism has been dubbed a "spinnetwork quantum simlator" by Rasetti and collaborators [67, 68]. In the next section we show how certain natural deformations [39] of Penrose spin networks [72] can be used to produce these unitary representations of the Artin braid group and the corresponding models for anyonic topological quantum computation.

\section{Spin Networks and Temperley-Lieb Recou- pling Theory}

In this section we discuss a combinatorial construction for spin networks that generalizes the original construction of Roger Penrose. The result of this generalization is a structure that satisfies all the properties of a graphical TQFT as described in the previous section, and specializes to classical angular momentum recoupling theory in the limit of its basic variable. The construction 
is based on the properties of the bracket polynomial (as already described in Section 4). A complete description of this theory can be found in the book "Temperley-Lieb Recoupling Theory and Invariants of Three-Manifolds" by Kauffman and Lins [39].

The " $q$-deformed" spin networks that we construct here are based on the bracket polynomial relation. View Figure 32 and Figure 33.
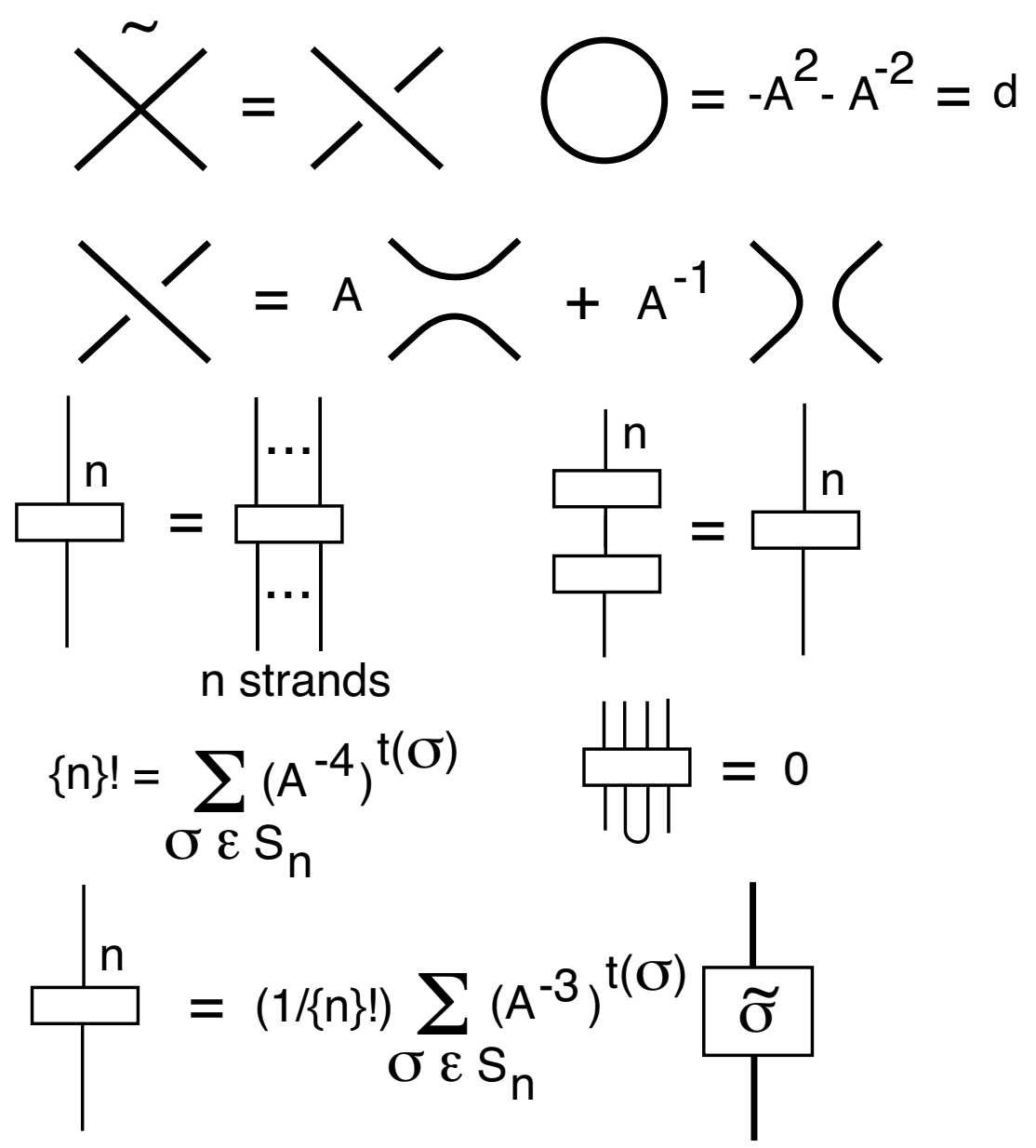

Figure 32 - Basic Projectors 


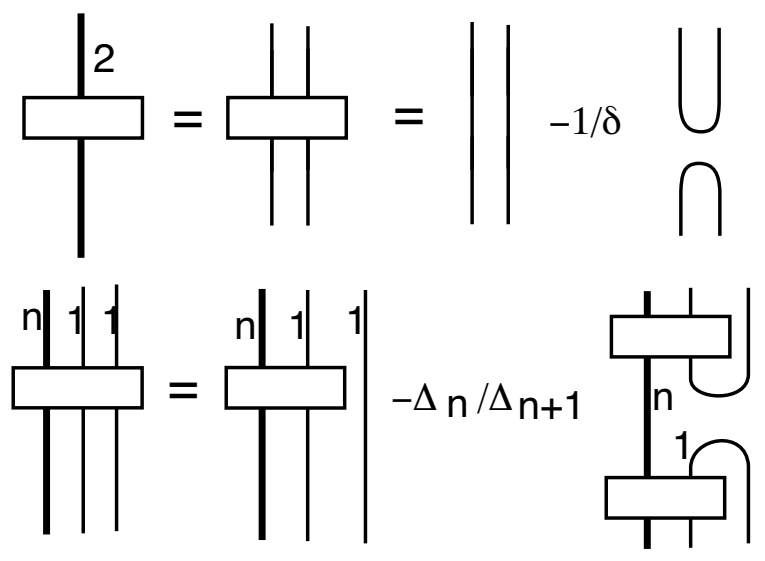

$$
\begin{aligned}
& \Delta_{-1}=0 \quad \Delta_{0}=1 \\
& \Delta_{\mathrm{n}+1}=\delta \Delta_{\mathrm{n}}-\Delta_{\mathrm{n}-1}
\end{aligned}
$$

Figure 33 - Two Strand Projector

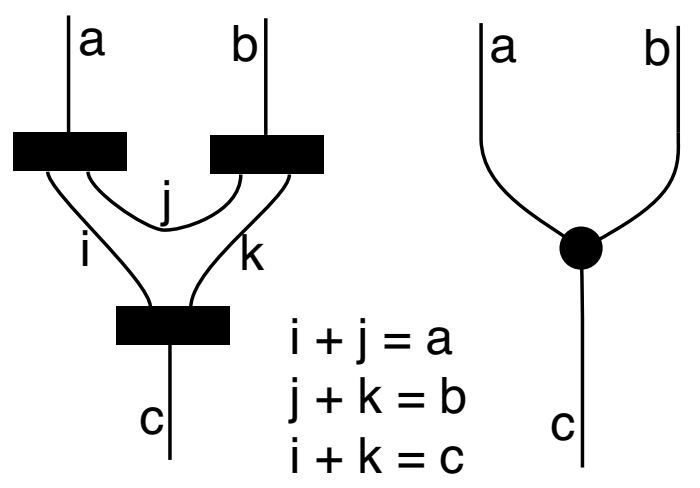

\section{Figure 34 -Vertex}

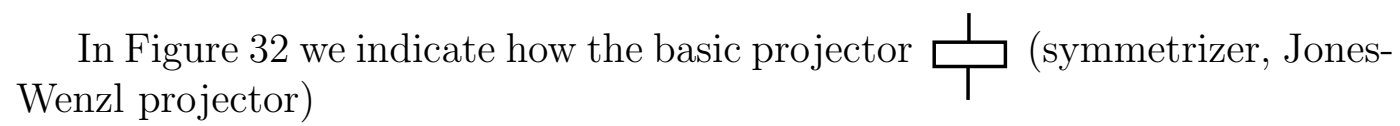


is constructed on the basis of the bracket polynomial expansion. In this technology a symmetrizer is a sum of tangles on $n$ strands (for a chosen integer $n$ ). The tangles are made by summing over braid lifts of permutations in the symmetric group on $n$ letters, as indicated in Figure 32. Each elementary braid is then expanded by the bracket polynomial relation as indicated in Figure 32 so that the resulting sum consists of flat tangles without any crossings (these can be viewed as elements in the Temperley-Lieb algebra). The projectors have the property that the concatenation of a projector with itself is just that projector, and if you tie two lines on the top or the bottom of a projector together, then the evaluation is zero. This general definition of projectors is very useful for this theory. The two-strand projector is shown in Figure 33. Here the formula for that projector is particularly simple. It is the sum of two parallel arcs and two turn-around arcs (with coefficient $-1 / d$, with $d=-A^{2}-A^{-2}$ is the loop value for the bracket polynomial. Figure 33 also shows the recursion formula for the general projector. This recursion formula is due to Jones and Wenzl and the projector in this form, developed as a sum in the Temperley-Lieb algebra (see Section 5 of this paper), is usually known as the Jones-Wenzl projector.

The projectors are combinatorial analogs of irreducible representations of a group (the original spin nets were based on $S U(2)$ and these deformed nets are based on the corresponding quantum group to $\mathrm{SU}(2)$ ). As such the reader can think of them as "particles". The interactions of these particles are governed by how they can be tied together into three-vertices. See Figure 34. In Figure 34 we show how to tie three projectors, of $a, b, c$ strands respectively, together to form a three-vertex. In order to accomplish this interaction, we must share lines between them as shown in that figure so that there are non-negative integers $i, j, k$ so that $a=i+j, b=j+k, c=i+k$. This is equivalent to the condition that $a+b+c$ is even and that the sum of any two of $a, b, c$ is greater than or equal to the third. For example $a+b \geq c$. One can think of the vertex as a possible particle interaction where $[a]$ and $[b]$ interact to produce $[c]$. That is, any two of the legs of the vertex can be regarded as interacting to produce the third leg.

There is a basic orthogonality of three vertices as shown in Figure 35. Here if we tie two three-vertices together so that they form a "bubble" in the middle, then the resulting network with labels $a$ and $b$ on its free ends is a multiple of an $a$-line (meaning a line with an $a$-projector on it) or zero (if $a$ is not equal to $b$ ). The multiple is compatible with the results of closing the diagram in the equation of Figure 35 so the two free ends are identified with one another. On closure, as shown in the figure, the left hand side of the equation becomes a Theta graph and the right hand side becomes a multiple of a "delta" where 
$\Delta_{a}$ denotes the bracket polynomial evaluation of the $a$-strand loop with a projector on it. The $\Theta(a, b, c)$ denotes the bracket evaluation of a theta graph made from three trivalent vertices and labeled with $a, b, c$ on its edges.

There is a recoupling formula in this theory in the form shown in Figure 36. Here there are "6-j symbols", recoupling coefficients that can be expressed, as shown in Figure 36, in terms of tetrahedral graph evaluations and theta graph evaluations. The tetrahedral graph is shown in Figure 37. One derives the formulas for these coefficients directly from the orthogonality relations for the trivalent vertices by closing the left hand side of the recoupling formula and using orthogonality to evaluate the right hand side. This is illustrated in Figure 38. The reader should be advised that there are specific calculational formulas for the theta and tetrahedral nets. These can be found in [39]. Here we are indicating only the relationships and external logic of these objects.

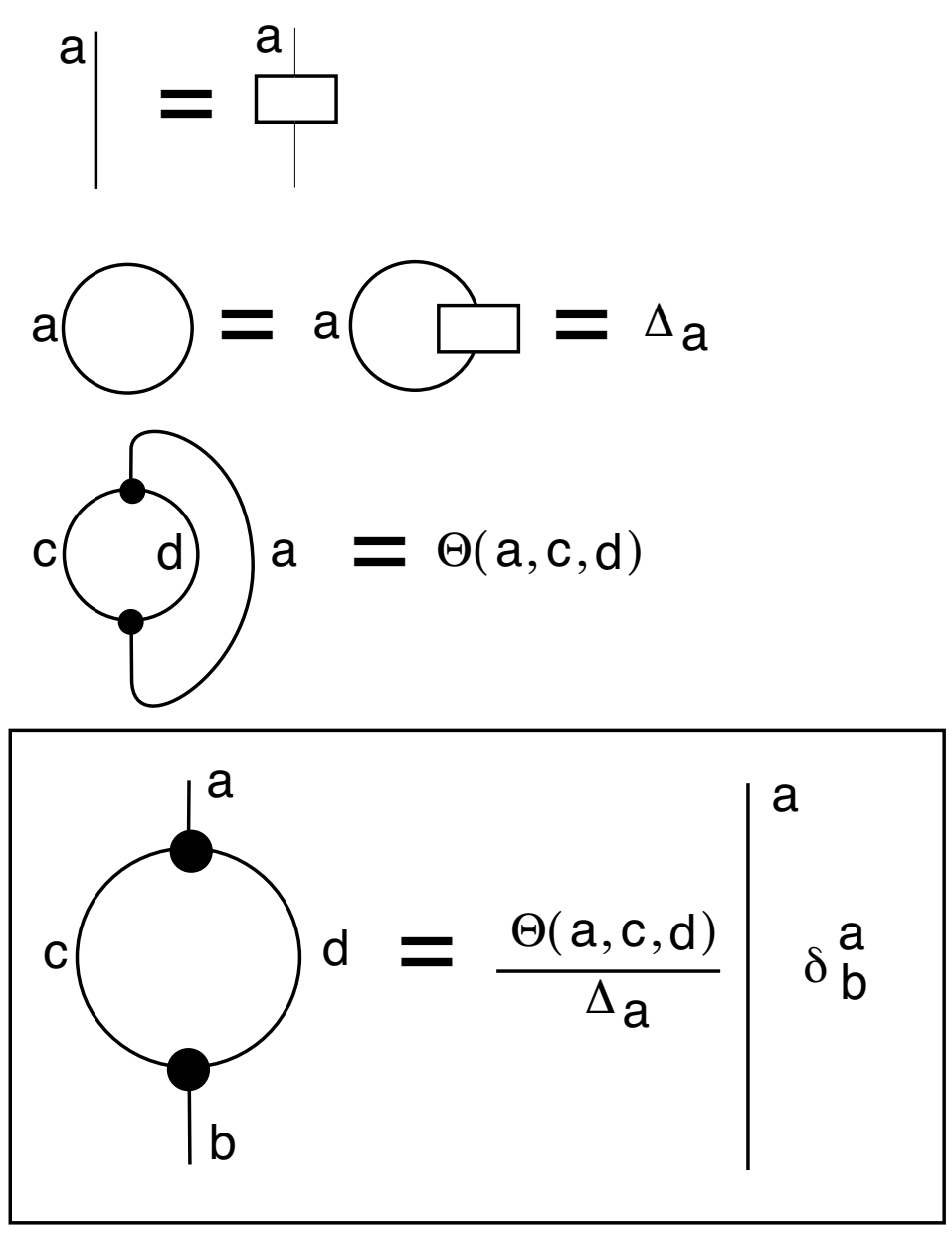

Figure 35 - Orthogonality of Trivalent Vertices 


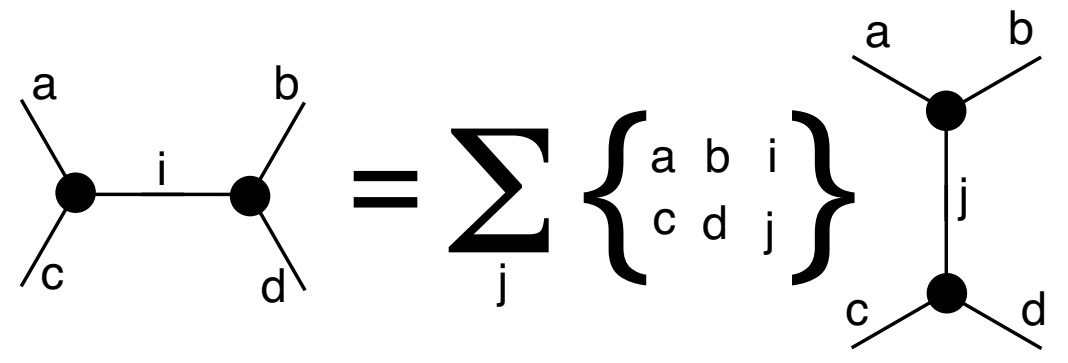

Figure 36 - Recoupling Formula

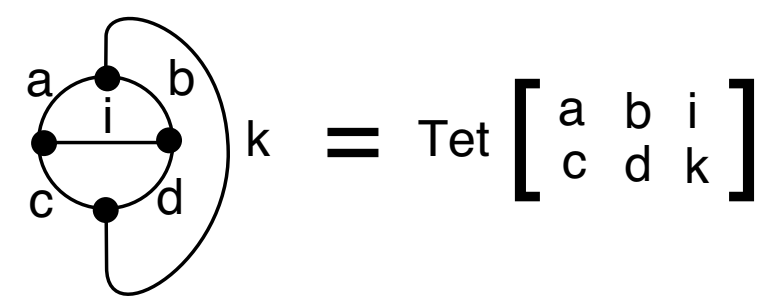

Figure 37 - Tetrahedron Network

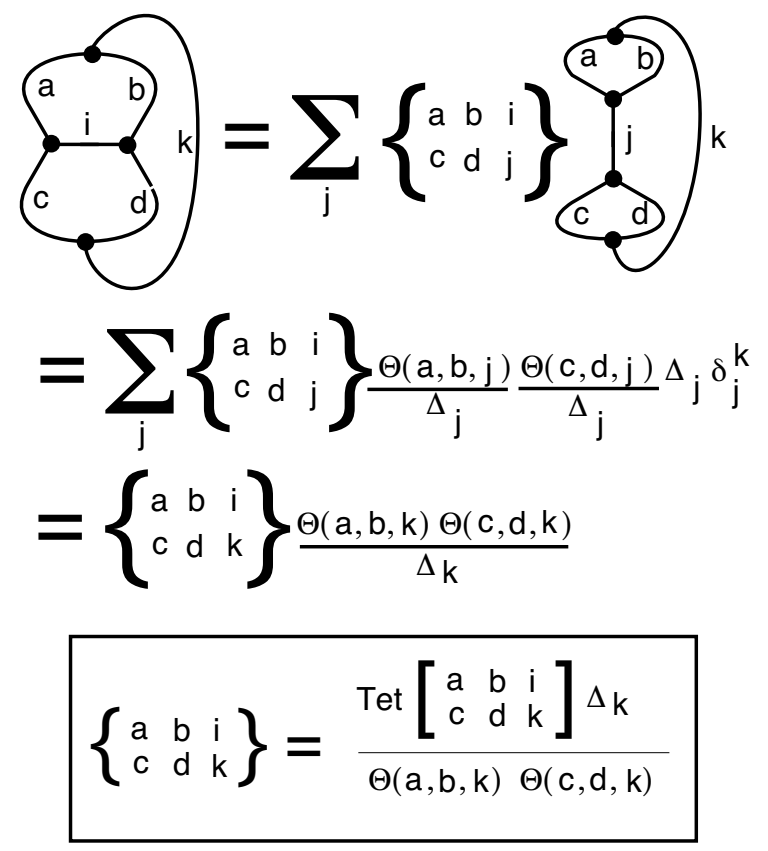

Figure 38 - Tetrahedron Formula for Recoupling Coefficients 
Finally, there is the braiding relation, as illustrated in Figure 36.

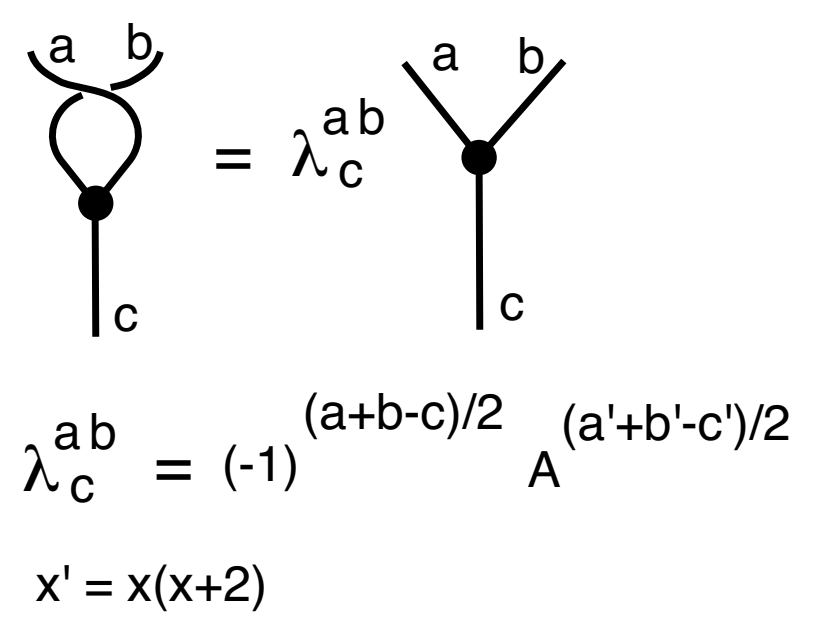

Figure 39 - Local Braiding Formula

With the braiding relation in place, this $q$-deformed spin network theory satisfies the pentagon, hexagon and braiding naturality identities needed for a topological quantum field theory. All these identities follow naturally from the basic underlying topological construction of the bracket polynomial. One can apply the theory to many different situations.

\subsection{Evaluations}

In this section we discuss the structure of the evaluations for $\Delta_{n}$ and the theta and tetrahedral networks. We refer to [39] for the details behind these formulas. Recall that $\Delta_{n}$ is the bracket evaluation of the closure of the $n$ strand projector, as illustrated in Figure 35. For the bracket variable $A$, one finds that

$$
\Delta_{n}=(-1)^{n} \frac{A^{2 n+2}-A^{-2 n-2}}{A^{2}-A^{-2}} .
$$

One sometimes writes the quantum integer

$$
[n]=(-1)^{n-1} \Delta_{n-1}=\frac{A^{2 n}-A^{-2 n}}{A^{2}-A^{-2}} .
$$

If

$$
A=e^{i \pi / 2 r}
$$


where $r$ is a positive integer, then

$$
\Delta_{n}=(-1)^{n} \frac{\sin ((n+1) \pi / r)}{\sin (\pi / r)} .
$$

Here the corresponding quantum integer is

$$
[n]=\frac{\sin (n \pi / r)}{\sin (\pi / r)}
$$

Note that $[n+1]$ is a positive real number for $n=0,1,2, \ldots r-2$ and that $[r-1]=0$.

The evaluation of the theta net is expressed in terms of quantum integers by the formula

$$
\Theta(a, b, c)=(-1)^{m+n+p} \frac{[m+n+p+1] ![n] ![m] ![p] !}{[m+n] ![n+p] ![p+m] !}
$$

where

$$
a=m+p, b=m+n, c=n+p .
$$

Note that

$$
(a+b+c) / 2=m+n+p .
$$

When $A=e^{i \pi / 2 r}$, the recoupling theory becomes finite with the restriction that only three-vertices (labeled with $a, b, c$ ) are admissible when $a+b+c \leq$ $2 r-4$. All the summations in the formulas for recoupling are restricted to admissible triples of this form.

\subsection{Symmetry and Unitarity}

The formula for the recoupling coefficients given in Figure 38 has less symmetry than is actually inherent in the structure of the situation. By multiplying all the vertices by an appropriate factor, we can reconfigure the formulas in this theory so that the revised recoupling transformation is orthogonal, in the sense that its transpose is equal to its inverse. This is a very useful fact. It means that when the resulting matrices are real, then the recoupling transformations are unitary. We shall see particular applications of this viewpoint later in the paper. 
Figure 40 illustrates this modification of the three-vertex. Let $\operatorname{Vert}[a, b, c]$ denote the original 3-vertex of the Temperley-Lieb recoupling theory. Let ModVert $[a, b, c]$ denote the modified vertex. Then we have the formula

$$
\operatorname{ModVert}[a, b, c]=\frac{\sqrt{\sqrt{\Delta_{a} \Delta_{b} \Delta_{c}}}}{\sqrt{\Theta(a, b, c)}} \operatorname{Vert}[a, b, c] .
$$

Lemma. For the bracket evaluation at the root of unity $A=e^{i \pi / 2 r}$ the factor

$$
f(a, b, c)=\frac{\sqrt{\sqrt{\Delta_{a} \Delta_{b} \Delta_{c}}}}{\sqrt{\Theta(a, b, c)}}
$$

is real, and can be taken to be a positive real number for $(a, b, c)$ admissible (i.e. $a+b+c \leq 2 r-4$ ).

Proof. By the results from the previous subsection,

$$
\Theta(a, b, c)=(-1)^{(a+b+c) / 2} \hat{\Theta}(a, b, c)
$$

where $\hat{\Theta}(a, b, c)$ is positive real, and

$$
\Delta_{a} \Delta_{b} \Delta_{c}=(-1)^{(a+b+c)}[a+1][b+1][c+1]
$$

where the quantum integers in this formula can be taken to be positive real. It follows from this that

$$
f(a, b, c)=\sqrt{\frac{\sqrt{[a+1][b+1][c+1]}}{\hat{\Theta}(a, b, c)}},
$$

showing that this factor can be taken to be positive real.

In Figure 41 we show how this modification of the vertex affects the nonzero term of the orthogonality of trivalent vertices (compare with Figure 35). We refer to this as the "modified bubble identity." The coefficient in the modified bubble identity is

$$
\sqrt{\frac{\Delta_{b} \Delta_{c}}{\Delta_{a}}}=(-1)^{(b+c-a) / 2} \sqrt{\frac{[b+1][c+1]}{[a+1]}}
$$

where $(a, b, c)$ form an admissible triple. In particular $b+c-a$ is even and hence this factor can be taken to be real. 
We rewrite the recoupling formula in this new basis and emphasize that the recoupling coefficients can be seen (for fixed external labels $a, b, c, d$ ) as a matrix transforming the horizontal "double- $Y$ " basis to a vertically disposed double- $Y$ basis. In Figures 42, 43 and 44 we have shown the form of this transformation,using the matrix notation

$$
M[a, b, c, d]_{i j}
$$

for the modified recoupling coefficients. In Figure 42 we derive an explicit formula for these matrix elements. The proof of this formula follows directly from trivalent-vertex orthogonality (See Figures 35 and 38.), and is given in Figure 42. The result shown in Figure 42 and Figure 43 is the following formula for the recoupling matrix elements.

$$
M[a, b, c, d]_{i j}=\operatorname{ModTet}\left(\begin{array}{ccc}
a & b & i \\
c & d & j
\end{array}\right) / \sqrt{\Delta_{a} \Delta_{b} \Delta_{c} \Delta_{d}}
$$

where $\sqrt{\Delta_{a} \Delta_{b} \Delta_{c} \Delta_{d}}$ is short-hand for the product

$$
\begin{gathered}
\sqrt{\frac{\Delta_{a} \Delta_{b}}{\Delta_{j}}} \sqrt{\frac{\Delta_{c} \Delta_{d}}{\Delta_{j}}} \Delta_{j} \\
=(-1)^{(a+b-j) / 2}(-1)^{(c+d-j) / 2}(-1)^{j} \sqrt{\frac{[a+1][b+1]}{[j+1]}} \sqrt{\frac{[c+1][d+1]}{[j+1]}}[j+1] \\
=(-1)^{(a+b+c+d) / 2} \sqrt{[a+1][b+1][c+1][d+1]}
\end{gathered}
$$

In this form, since $(a, b, j)$ and $(c, d, j)$ are admissible triples, we see that this coeffient can be taken to be real, and its value is independent of the choice of $i$ and $j$. The matrix $M[a, b, c, d]$ is real-valued.

It follows from Figure 36 (turn the diagrams by ninety degrees) that

$$
M[a, b, c, d]^{-1}=M[b, d, a, c] .
$$

In Figure 45 we illustrate the formula

$$
M[a, b, c, d]^{T}=M[b, d, a, c] .
$$

It follows from this formula that

$$
M[a, b, c, d]^{T}=M[a, b, c, d]^{-1}
$$

Hence $M[a, b, c, d]$ is an orthogonal, real-valued matrix. 


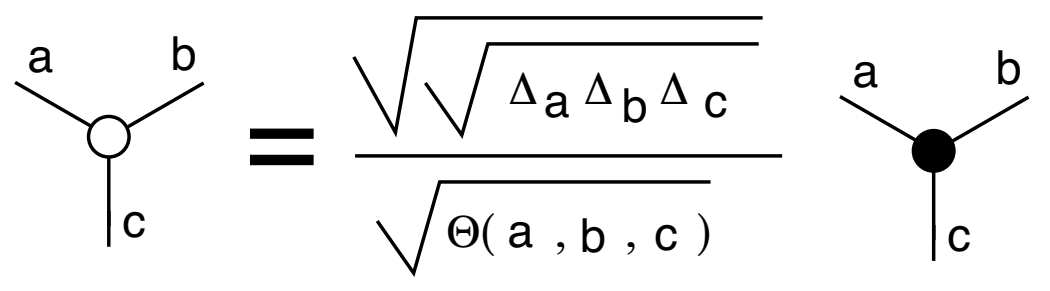

Figure 40 - Modified Three Vertex
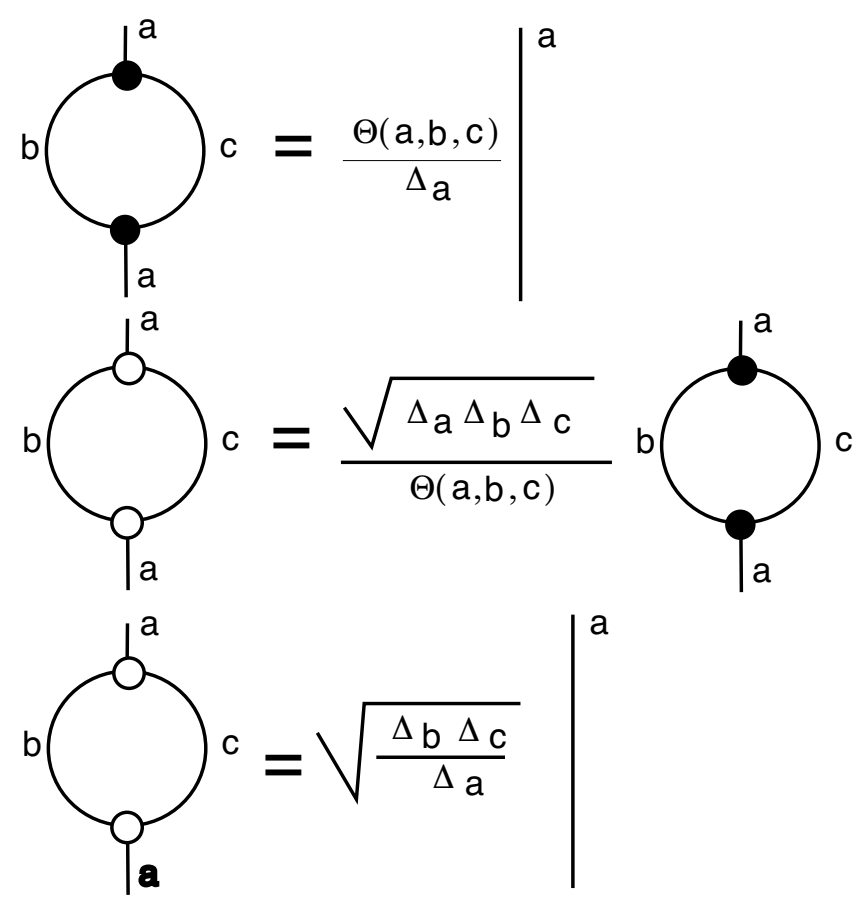

Figure 41 - Modified Bubble Identiy 


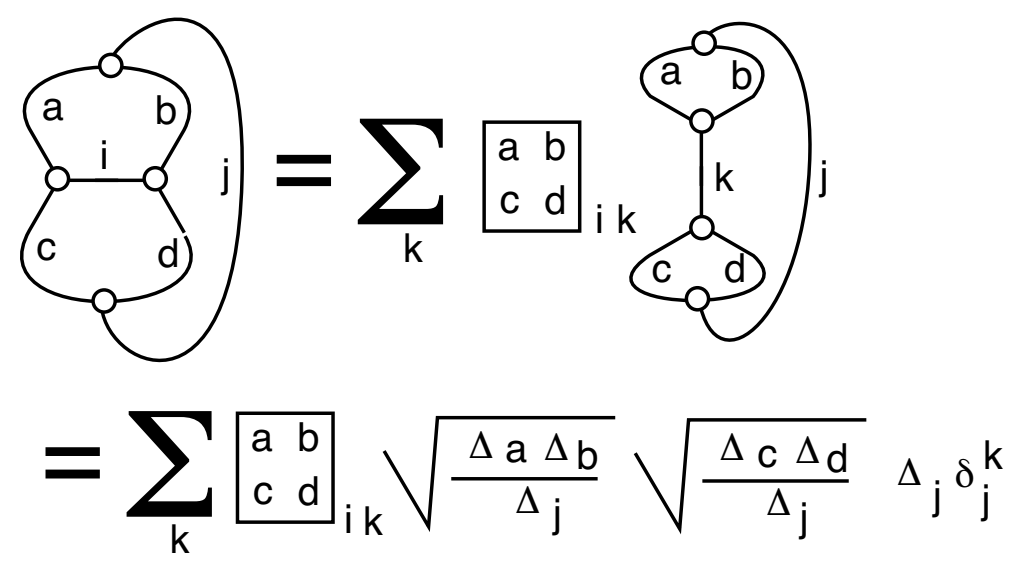

$$
=\left[\begin{array}{ll}
a & b \\
c & d
\end{array}\right]_{i j} \sqrt{\frac{\Delta a \Delta b}{\Delta j}} \sqrt{\frac{\Delta c \Delta_{d}}{\Delta_{j}}} \Delta_{j}
$$

$$
\frac{\left[\begin{array}{ll}
a \\
c & d
\end{array}\right]_{i j}=\frac{\operatorname{ModTet}\left[\begin{array}{lll}
a & b & i \\
c & d & j
\end{array}\right]}{\sqrt{\frac{\Delta a \Delta_{b} \Delta_{b} \Delta_{c} \Delta_{d}}{\Delta_{j}}} \sqrt{\frac{\Delta_{c} \Delta_{d}}{\Delta_{j}}} \Delta_{j}}}{\sqrt{\Delta_{d}^{b}}}
$$

Figure 42 - Derivation of Modified Recoupling Coefficients 


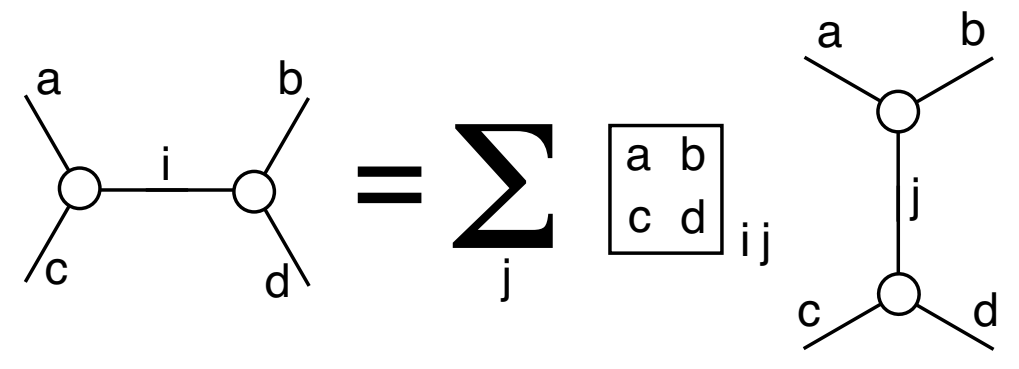

Figure 43 - Modified Recoupling Formula

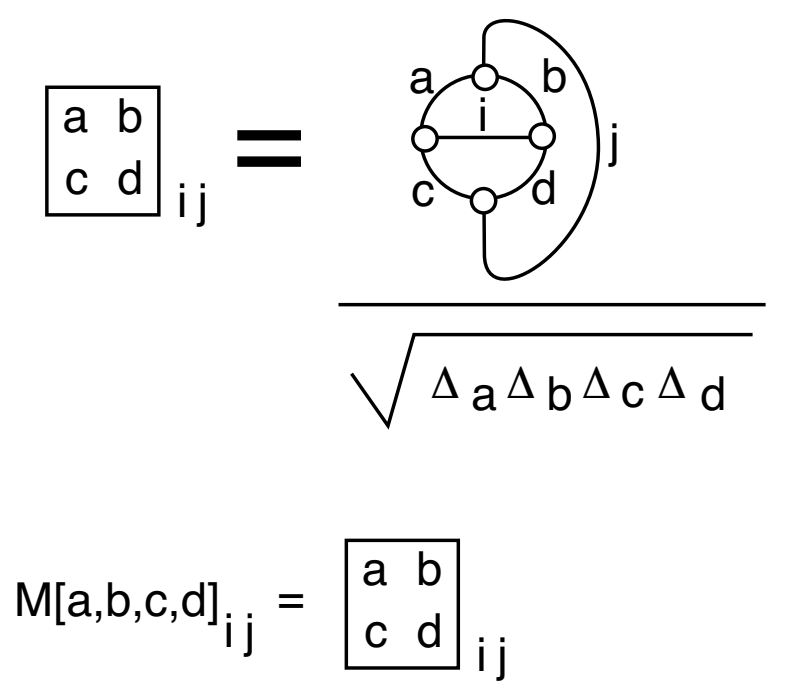

Figure 44 - Modified Recoupling Matrix 


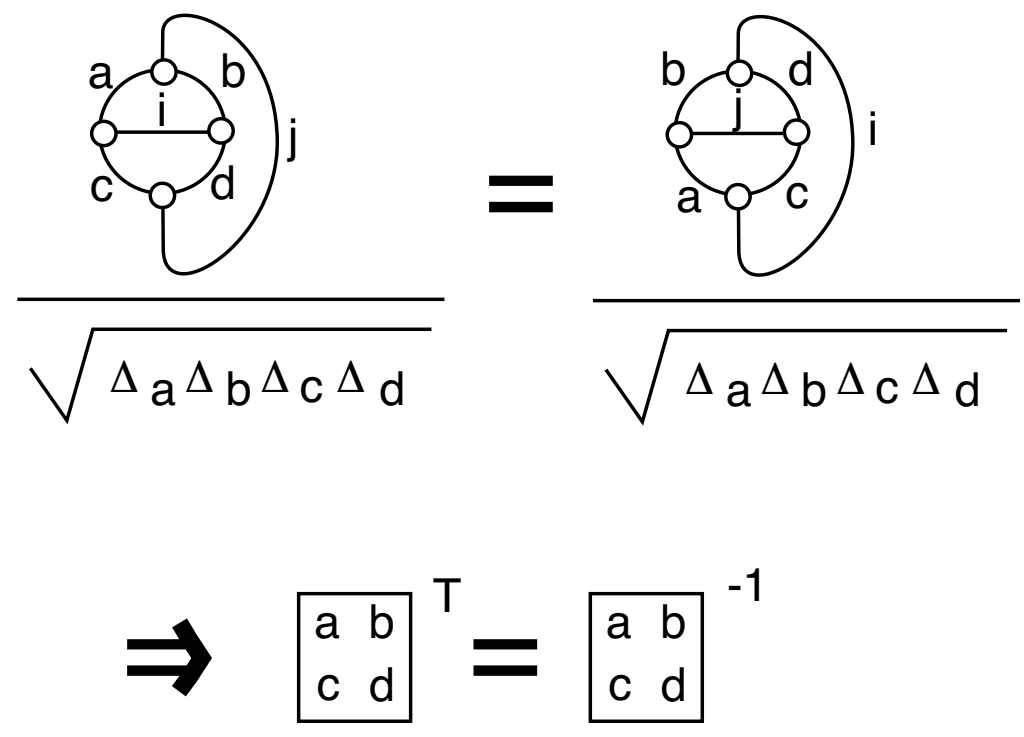

Figure 45 - Modified Matrix Transpose

Theorem. In the Temperley-Lieb theory we obtain unitary (in fact real orthogonal) recoupling transformations when the bracket variable $A$ has the form $A=e^{i \pi / 2 r}$ for $r$ a positive integer. Thus we obtain families of unitary representations of the Artin braid group from the recoupling theory at these roots of unity.

Proof. The proof is given the discussion above.

In Section 9 we shall show explictly how these methods work in the case of the Fibonacci model where $A=e^{3 i \pi / 5}$.

\section{Fibonacci Particles}

In this section and the next we detail how the Fibonacci model for anyonic quantum computing $[62,73]$ can be constructed by using a version of the twostranded bracket polynomial and a generalization of Penrose spin networks. This is a fragment of the Temperly-Lieb recoupling theory [39]. We already gave in the preceding sections a general discussion of the theory of spin networks and their relationship with quantum computing. 
The Fibonacci model is a TQFT that is based on a single "particle" with two states that we shall call the marked state and the unmarked state. The particle in the marked state can interact with itself either to produce a single particle in the marked state, or to produce a single particle in the unmarked state. The particle in the unmarked state has no influence in interactions (an unmarked state interacting with any state $S$ yields that state $S$ ). One way to indicate these two interactions symbolically is to use a box,for the marked state and a blank space for the unmarked state. Then one has two modes of interaction of a box with itself:

1. Adjacency:

and

2. Nesting:

With this convention we take the adjacency interaction to yield a single box, and the nesting interaction to produce nothing:

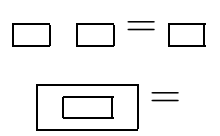

We take the notational opportunity to denote nothing by an asterisk $\left(^{*}\right)$. The syntatical rules for operating the asterisk are Thus the asterisk is a stand-in for no mark at all and it can be erased or placed wherever it is convenient to do so. Thus

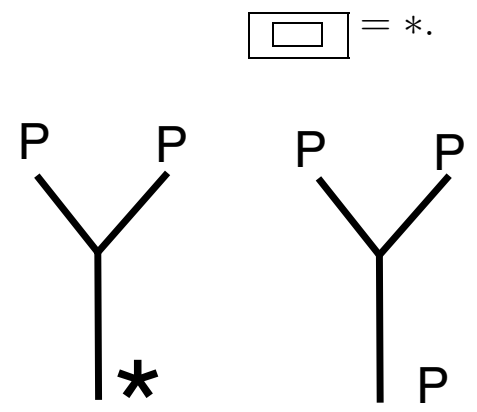

Figure 46 - Fibonacci Particle Interaction

We shall make a recoupling theory based on this particle, but it is worth noting some of its purely combinatorial properties first. The arithmetic of combining boxes (standing for acts of distinction) according to these rules has been studied and formalized in [82] and correlated with Boolean algebra and classical logic. Here within and next to are ways to refer to the two 
sides delineated by the given distinction. From this point of view, there are two modes of relationship (adjacency and nesting) that arise at once in the presence of a distinction.
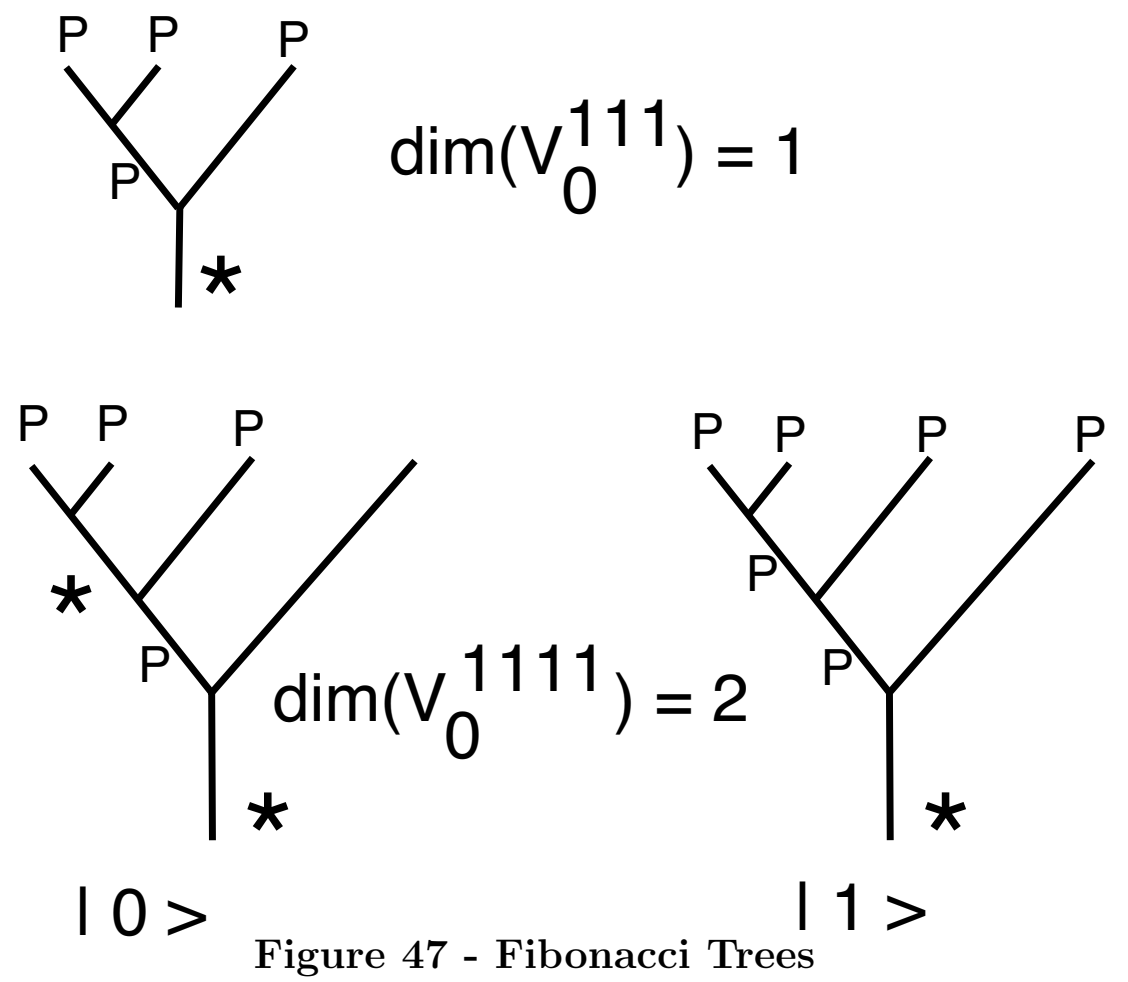

From here on we shall denote the Fibonacii particle by the letter $P$. Thus the two possible interactions of $P$ with itself are as follows.

1. $P, P \longrightarrow *$

2. $P, P \longrightarrow P$

In Figure 47 we indicate in small tree diagrams the two possible interactions of the particle $P$ with itself. In the first interaction the particle vanishes, producing the asterix. In the second interaction the particle a single copy of $P$ is produced. These are the two basic actions of a single distinction relative to itself, and they constitute our formalism for this very elementary particle.

In Figure 47, we have indicated the different results of particle processes where we begin with a left-associated tree structure with three branches, all marked and then four branches all marked. In each case we demand that the particles interact successively to produce an unmarked particle in the end, at 
the root of the tree. More generally one can consider a left-associated tree with $n$ upward branches and one root. Let $T\left(a_{1}, a_{2}, \cdots, a_{n}: b\right)$ denote such a tree with particle labels $a_{1}, \cdots, a_{n}$ on the top and root label $b$ at the bottom of the tree. We consider all possible processes (sequences of particle interactions) that start with the labels at the top of the tree, and end with the labels at the bottom of the tree. Each such sequence is regarded as a basis vector in a complex vector space

$$
V_{b}^{a_{1}, a_{2}, \cdots, a_{n}}
$$

associated with the tree. In the case where all the labels are marked at the top and the bottom label is unmarked, we shall denote this tree by

$$
V_{0}^{111 \cdots 11}=V_{0}^{(n)}
$$

where $n$ denotes the number of upward branches in the tree. We see from Figure 47 that the dimension of $V_{0}^{(3)}$ is 1 , and that

$$
\operatorname{dim}\left(V_{0}^{(4)}\right)=2
$$

This means that $V_{0}^{(4)}$ is a natural candidate in this context for the two-qubit space.

Given the tree $T(1,1,1, \cdots, 1: 0)$ ( $n$ marked states at the top, an unmarked state at the bottom), a process basis vector in $V_{0}^{(n)}$ is in direct correspondence with a string of boxes and asterisks (1's and 0's) of length $n-2$ with no repeated asterisks and ending in a marked state. See Figure 47 for an illustration of the simplest cases. It follows from this that

$$
\operatorname{dim}\left(V_{0}^{(n)}\right)=f_{n-2}
$$

where $f_{k}$ denotes the $k$-th Fibonacci number:

$$
f_{0}=1, f_{1}=1, f_{2}=2, f_{3}=3, f_{4}=5, f_{5}=8, \cdots
$$

where

$$
f_{n+2}=f_{n+1}+f_{n}
$$

The dimension formula for these spaces follows from the fact that there are $f_{n}$ sequences of length $n-1$ of marked and unmarked states with no repetition of an unmarked state. This fact is illustrated in Figure 48. 


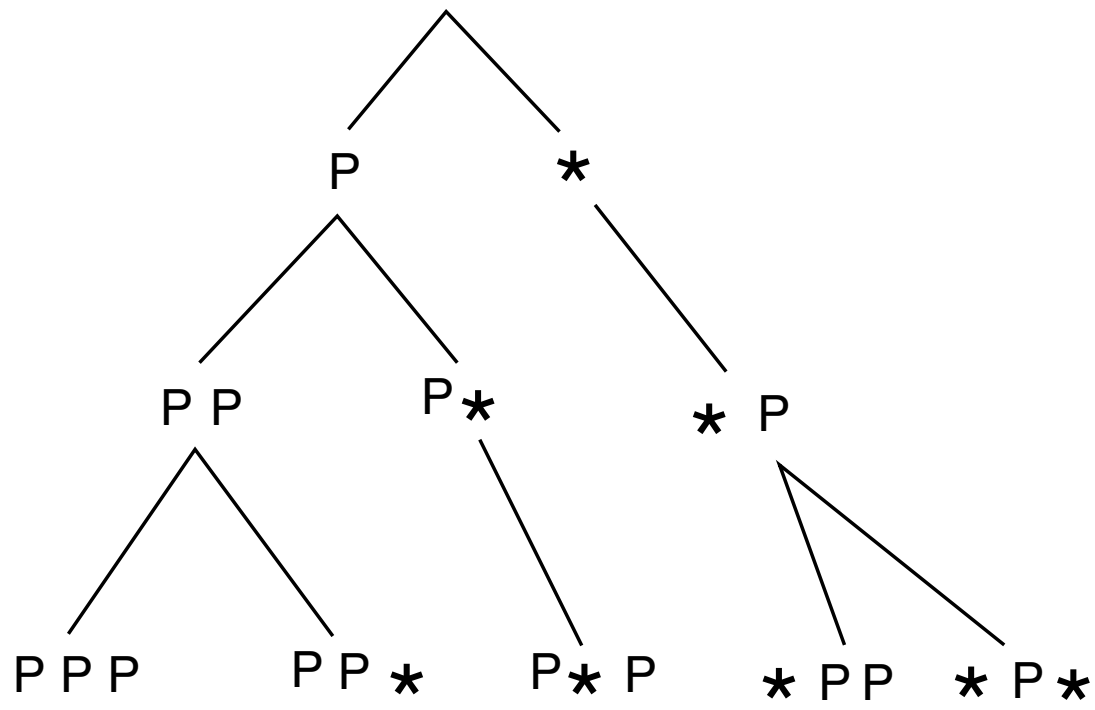

\title{
Tree of squences with no occurence of $\star \star$
}

\author{
Figure 48 - Fibonacci Sequence
}

\section{The Fibonacci Recoupling Model}

We now show how to make a model for recoupling the Fibonacci particle by using the Temperley Lieb recoupling theory and the bracket polynomial. Everything we do in this section will be based on the 2-projector, its properties and evaluations based on the bracket polynomial model for the Jones polynomial. While we have outlined the general recoupling theory based on the bracket polynomial in earlier sections of this paper, the present section is selfcontained, using only basic information about the bracket polyonmial, and the essential properties of the 2-projector as shown in Figure 49. In this figure we state the definition of the 2-projector, list its two main properties (the operator is idempotent and a self-attached strand yields a zero evaluation) and give diagrammatic proofs of these properties. 


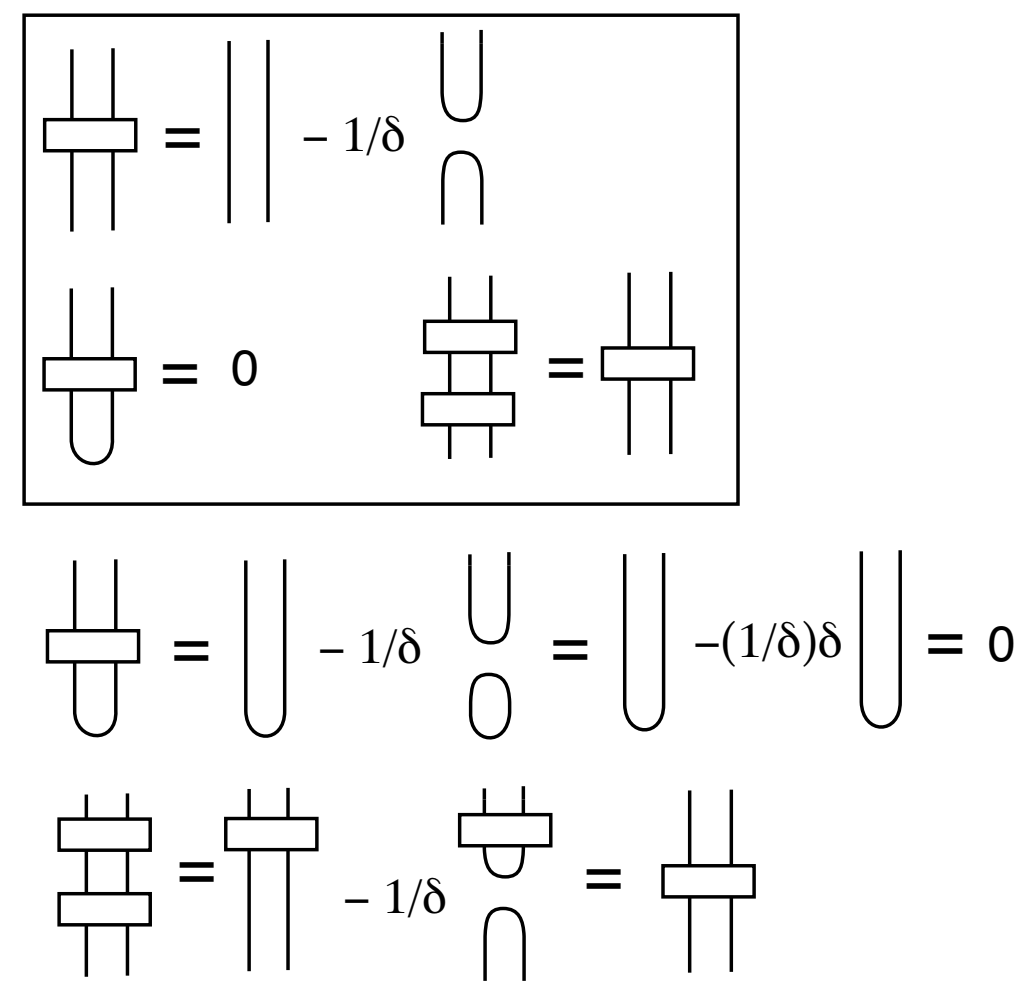

Figure 49 - The 2-Projector

In Figure 50, we show the essence of the Temperley-Lieb recoupling model for the Fibonacci particle. The Fibonaccie particle is, in this mathematical model, identified with the 2-projector itself. As the reader can see from Figure 50 , there are two basic interactions of the 2-projector with itself, one giving a 2-projector, the other giving nothing. This is the pattern of self-iteraction of the Fibonacci particle. There is a third possibility, depicted in Figure 50, where two 2-projectors interact to produce a 4 -projector. We could remark at the outset, that the 4-projector will be zero if we choose the bracket polynomial variable $A=e^{3 \pi / 5}$. Rather than start there, we will assume that the 4-projector is forbidden and deduce (below) that the theory has to be at this root of unity. 

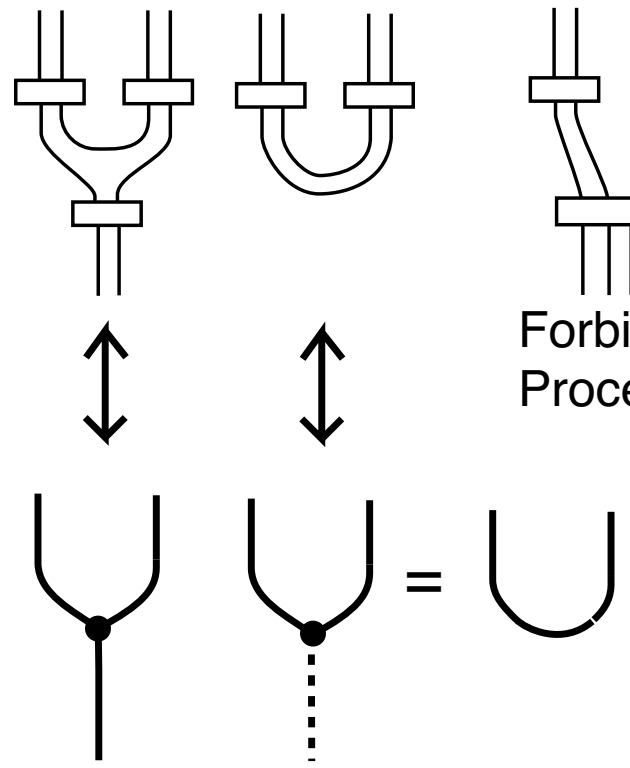

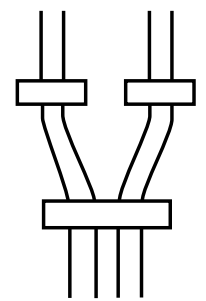

Forbidden Process

\section{Figure 50 - Fibonacci Particle as 2-Projector}

Note that in Figure 50 we have adopted a single strand notation for the particle interactions, with a solid strand corresponding to the marked particle, a dotted strand (or nothing) corresponding to the unmarked particle. A dark vertex indicates either an interaction point, or it may be used to indicate the single strand is shorthand for two ordinary strands. Remember that these are all shorthand expressions for underlying bracket polynomial calculations.

In Figures 51, 52, 53, 54, 55 and 56 we have provided complete diagrammatic calculations of all of the relevant small nets and evaluations that are useful in the two-strand theory that is being used here. The reader may wish to skip directly to Figure 57 where we determine the form of the recoupling coefficients for this theory. We will discuss the resulting algebra below.

For the reader who does not want to skip the next collection of figures, here is a guided tour. Figure 51 illustrates three three basic nets in case of two strands. These are the theta, delta and tetrahedron nets. In this figure we have shown the decomposition on the theta and delta nets in terms of 2projectors. The Tetrahedron net will be similarly decomposed in Figures 55 and 56. The theta net is denoted $\Theta$, the delta by $\Delta$, and the tetrahedron by $T$. In Figure 52 we illustrate how a pedant loop has a zero evaluation. In Figure 53 we use the identity in Figure 52 to show how an interior loop (formed by two trivalent vertices) can be removed and replaced by a factor of $\Theta / \Delta$. Note how, in this figure, line two proves that one network is a multiple of the other, while line three determines the value of the multiple by closing both nets. 
Figure 54 illustrates the explicit calculation of the delta and theta nets. The figure begins with a calculation of the result of closing a single strand of the 2-projector. The result is a single stand multiplied by $(\delta-1 / \delta)$ where $\delta=$ $-A^{2}-A^{-2}$, and $A$ is the bracket polynomial parameter. We then find that

$$
\Delta=\delta^{2}-1
$$

and

$$
\Theta=(\delta-1 / \delta)^{2} \delta-\Delta / \delta=(\delta-1 / \delta)\left(\delta^{2}-2\right)
$$

Figures 55 and 56 illustrate the calculation of the value of the tetrahedral network $T$. The reader should note the first line of Figure 55 where the tetradedral net is translated into a pattern of 2-projectors, and simplified. The rest of these two figures are a diagrammatic calculation, using the expansion formula for the 2-projector. At the end of Figure 56 we obtain the formula for the tetrahedron

$$
T=(\delta-1 / \delta)^{2}\left(\delta^{2}-2\right)-2 \Theta / \delta
$$

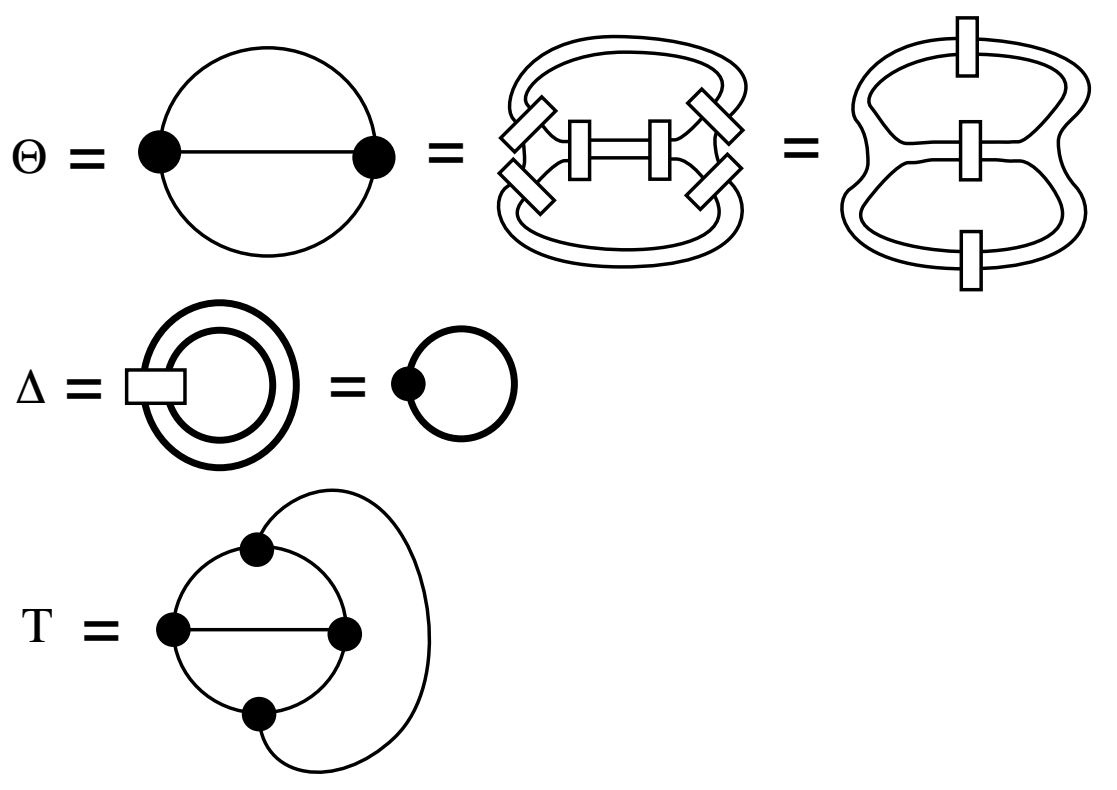

Figure 51 - Theta, Delta and Tetrahedron 


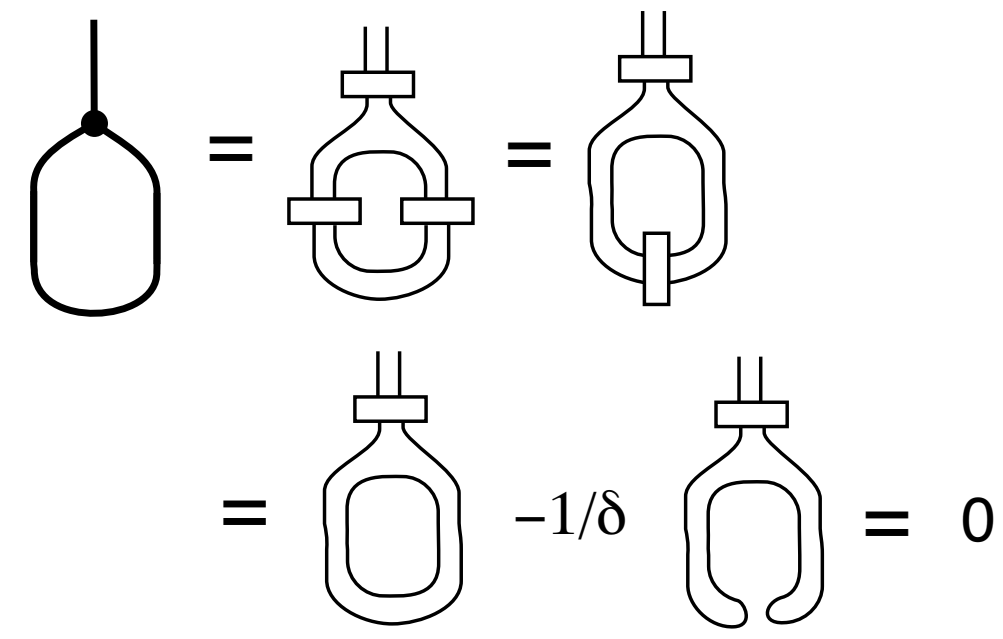

Figure 52 - LoopEvaluation-1
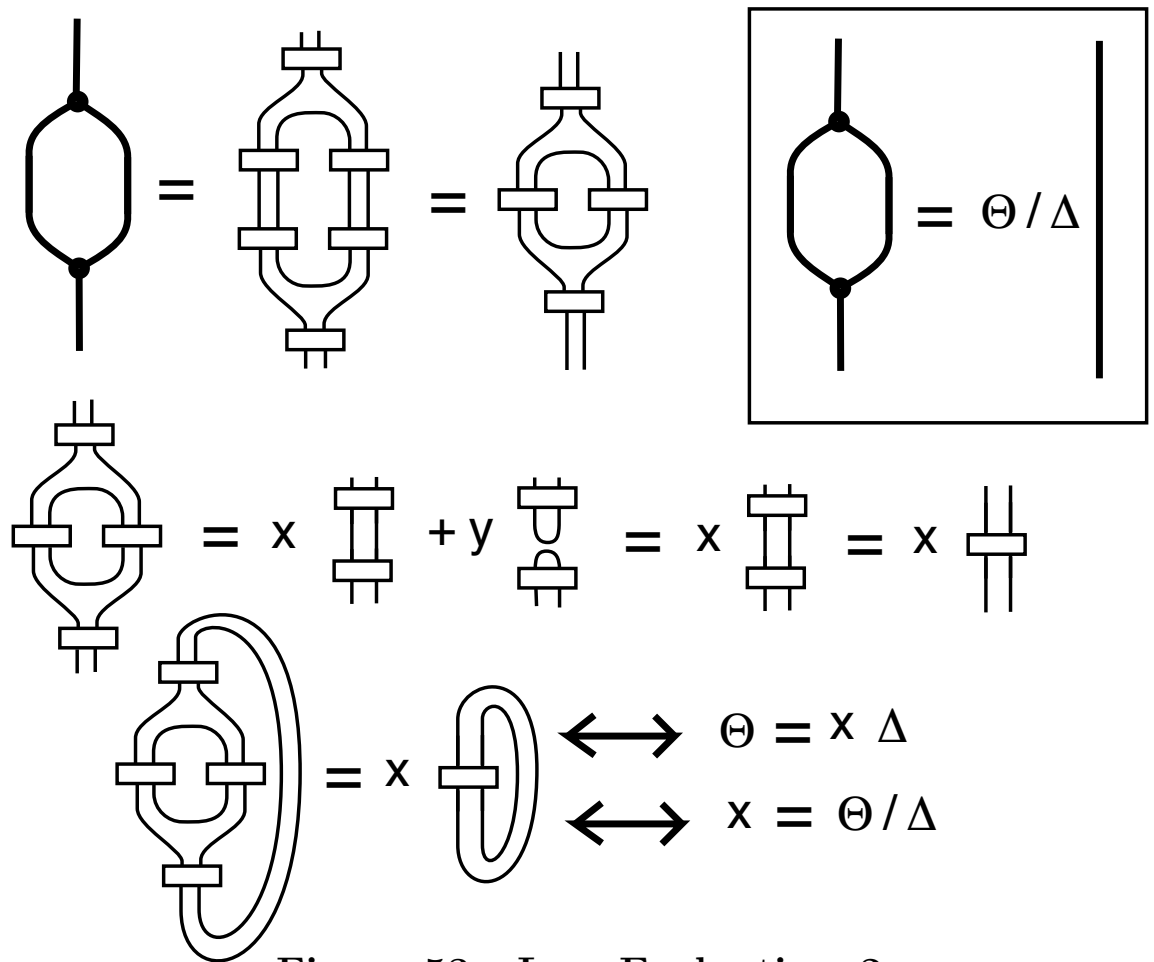

Figure 53 - LoopEvaluation-2 


$$
\begin{aligned}
& \downarrow=|O-1 / \delta \zeta=(\delta-1 / \delta)| \\
& \Delta=\frac{1}{\square}=(\delta-1 / \delta)(\delta-1 / \delta) \delta \\
& \Delta=\delta^{2}-1
\end{aligned}
$$

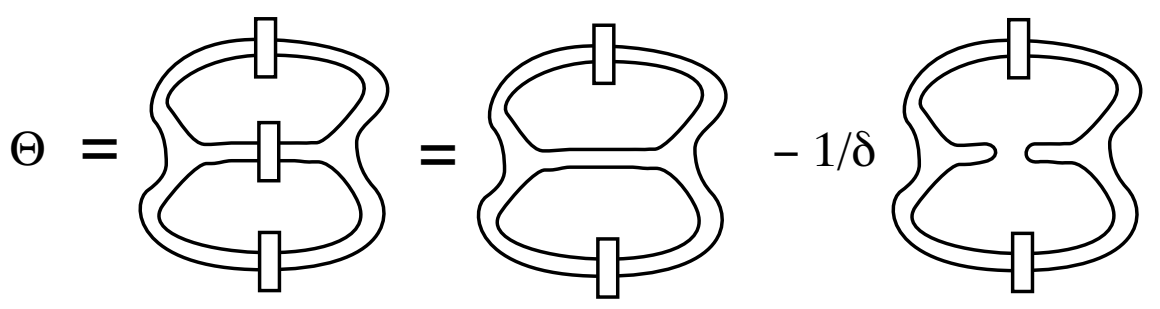

$$
\Theta=(\delta-1 / \delta)^{2} \delta-\Delta / \delta
$$

Figure 54 - Calculate Theta, Delta

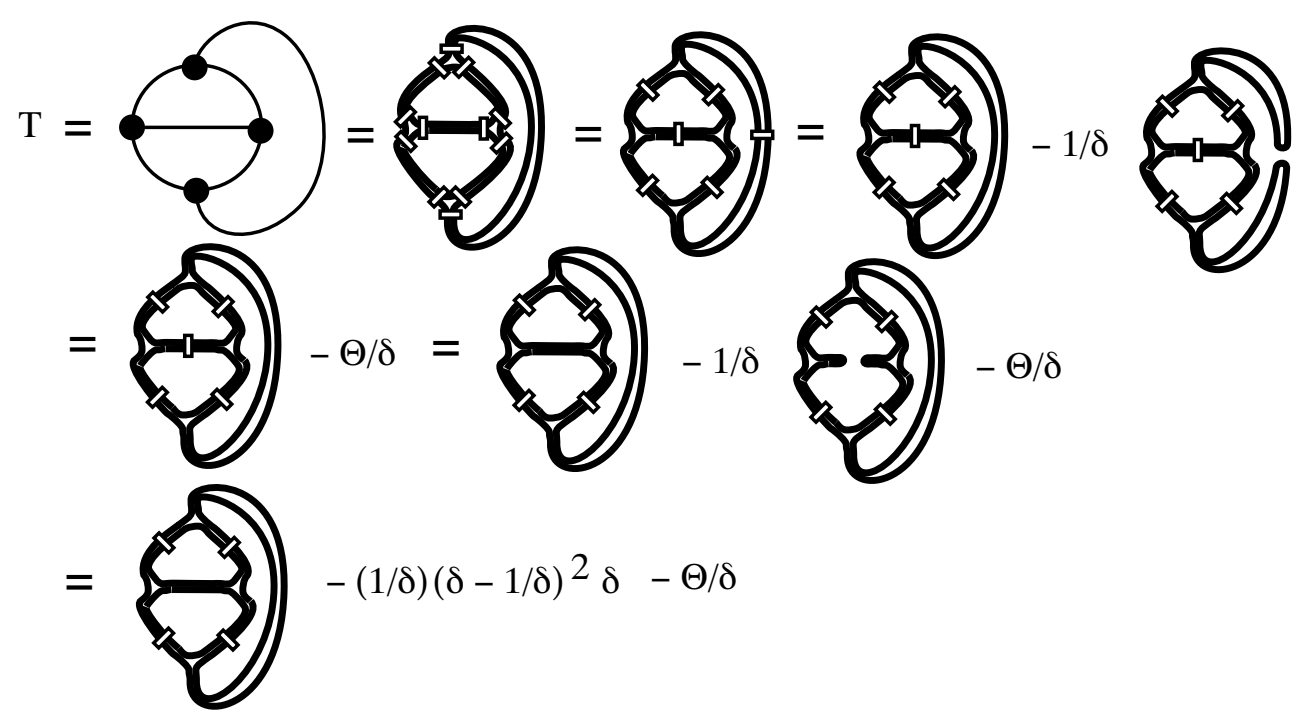

Figure 55 - Calculate Tetrahedron - 1 


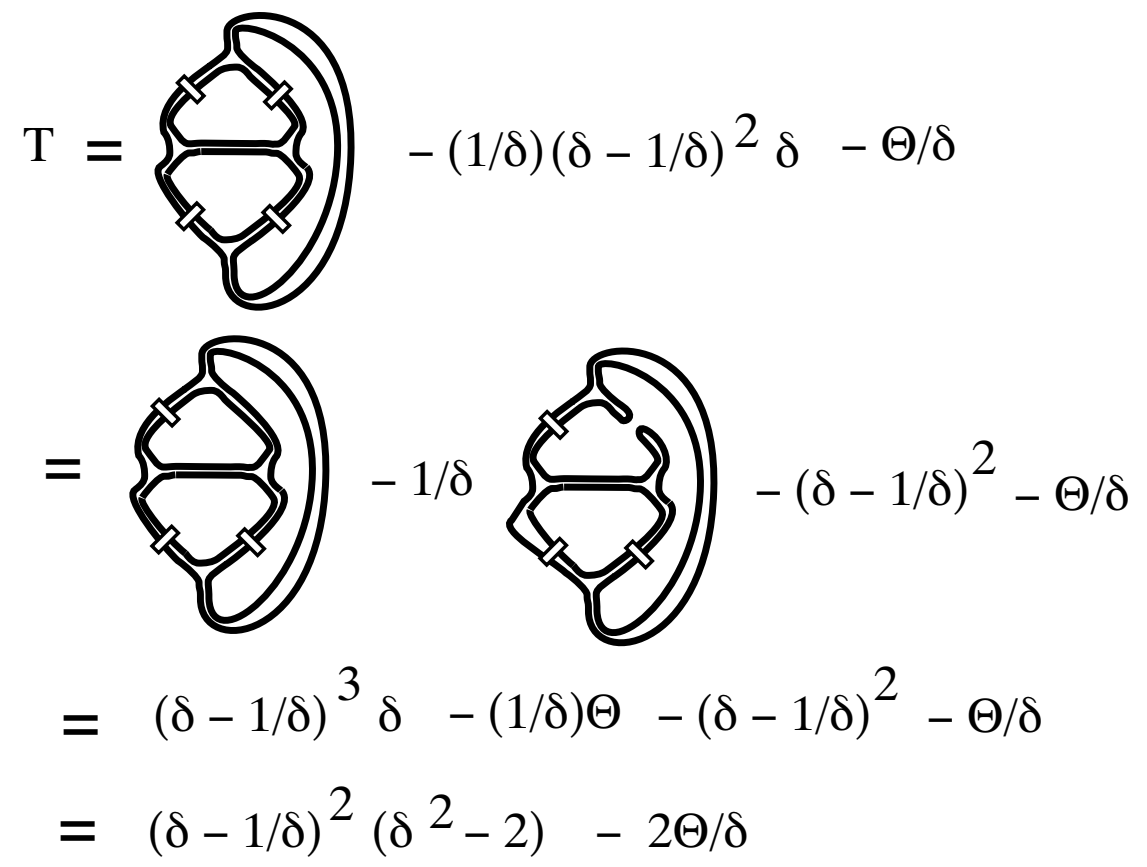

Figure 56 - Calculate Tetrahedron - 2

Figure 57 is the key calculation for this model. In this figure we assume that the recoupling formulas involve only 0 and 2 strands, with 0 corresponding to the null particle and 2 corresponding to the 2-projector. $(2+2=4$ is forbidden as in Figure 50.) From this assumption we calculate that the recoupling matrix is given by

$$
F=\left(\begin{array}{ll}
a & b \\
c & d
\end{array}\right)=\left(\begin{array}{cc}
1 / \Delta & \Delta / \Theta \\
\Theta / \Delta^{2} & T \Delta / \Theta^{2}
\end{array}\right)
$$




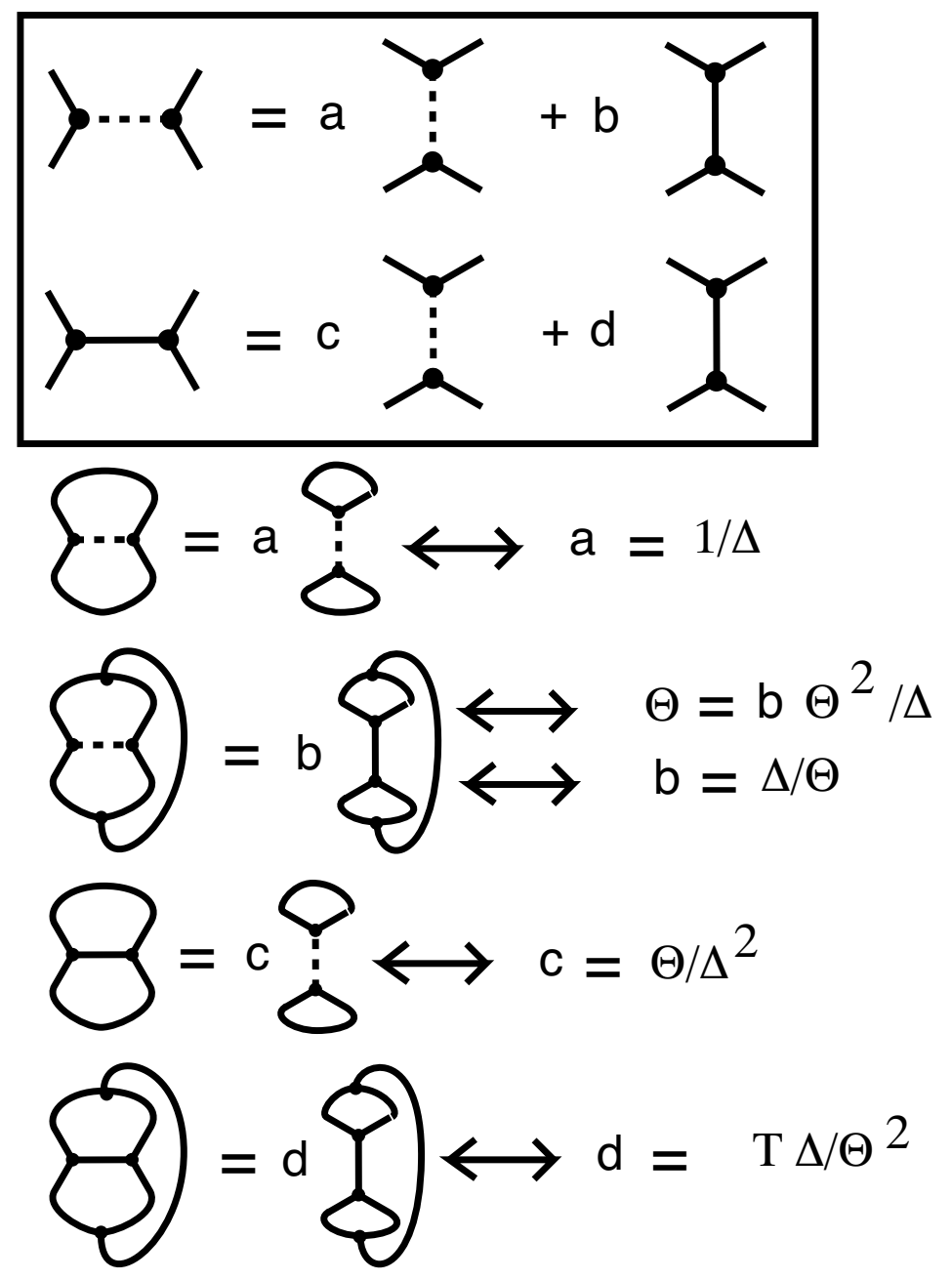

Figure 57 - Recoupling for 2-Projectors 

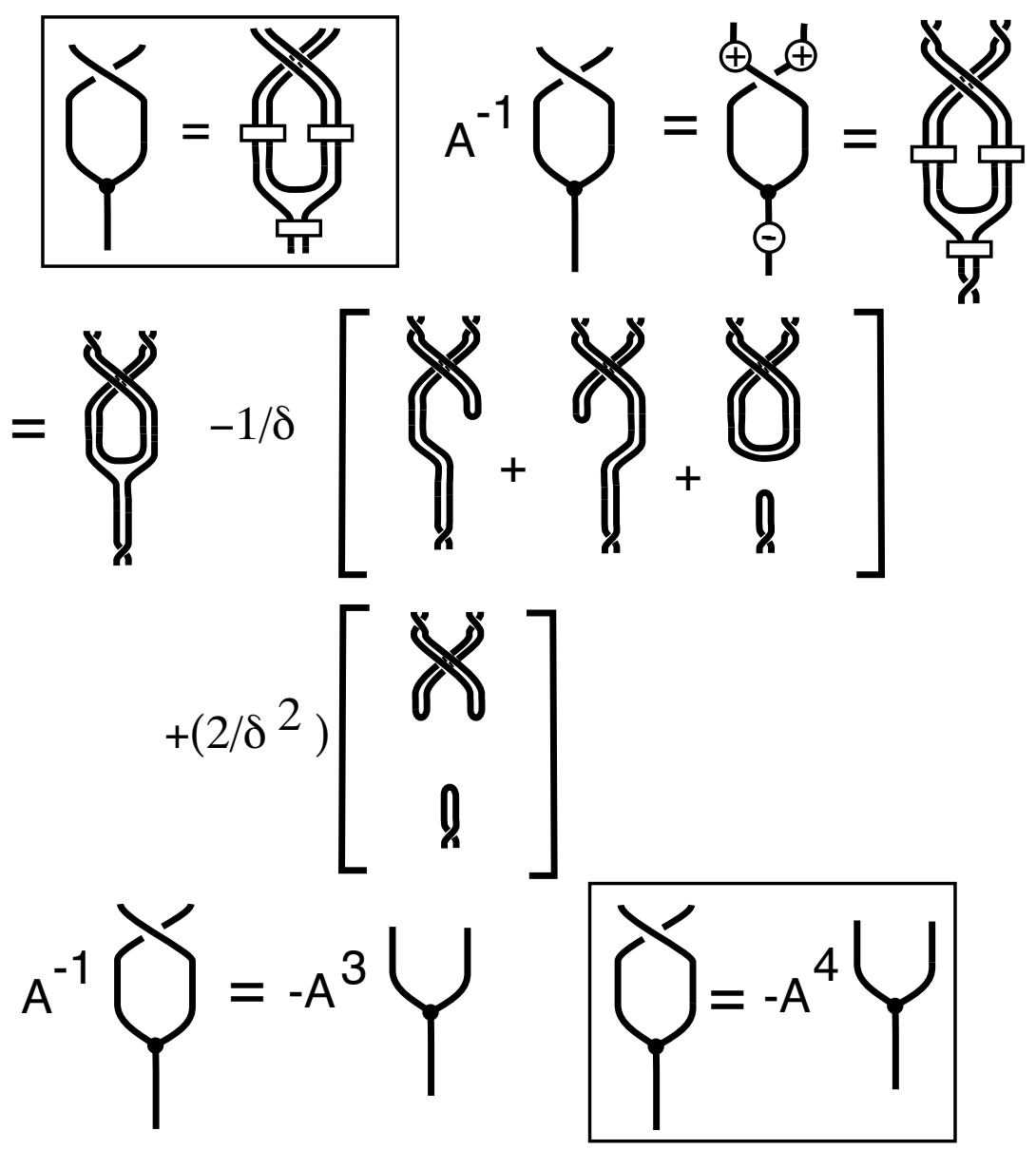

Figure 58 - Braiding at the Three-Vertex 


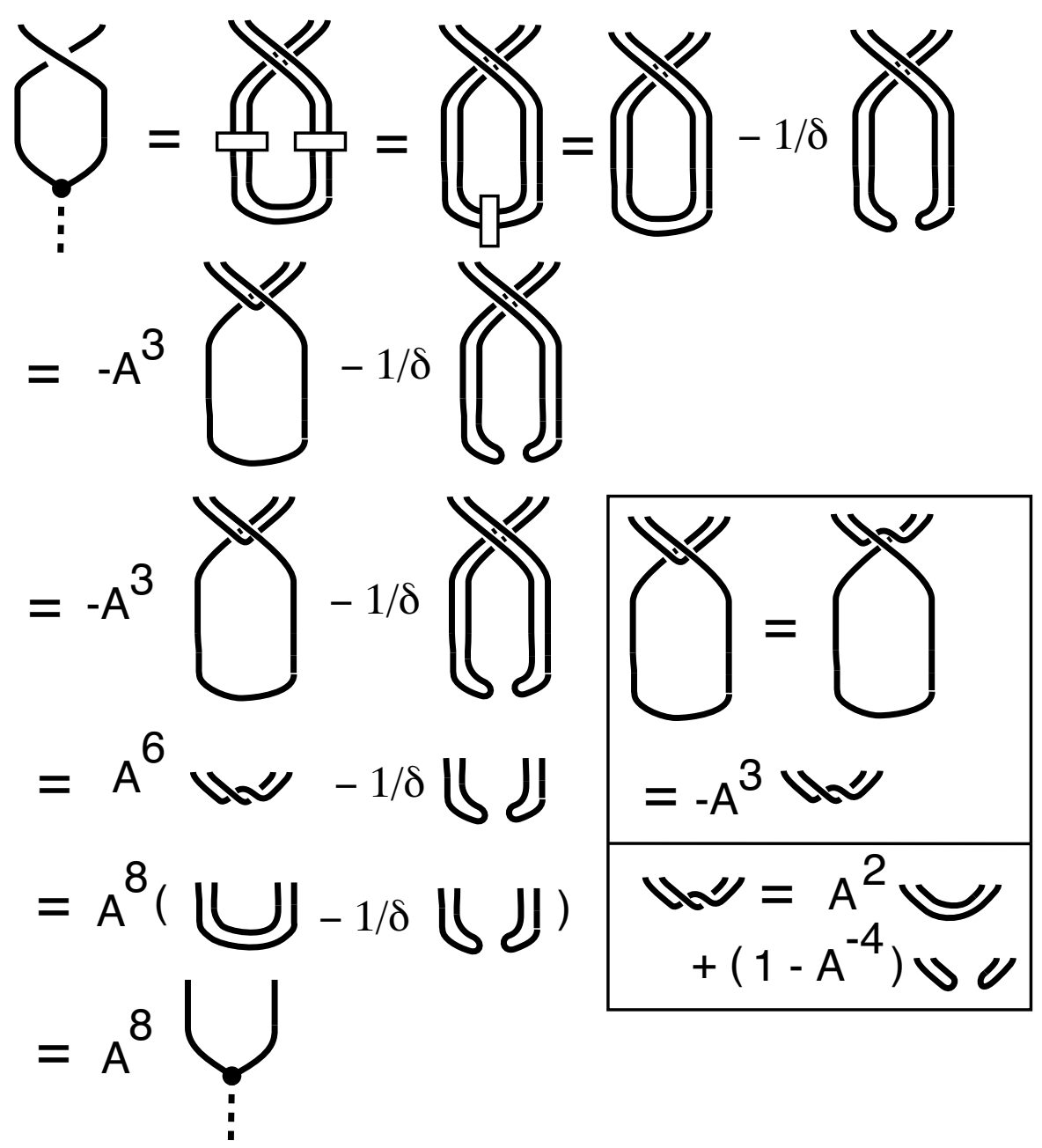

Figure 59 - Braiding at the Null-Three-Vertex

Figures 58 and 59 work out the exact formulas for the braiding at a three-vertex in this theory. When the 3-vertex has three marked lines, then the braiding operator is multiplication by $-A^{4}$, as in Figure 58 . When the 3 -vertex has two marked lines, then the braiding operator is multiplication by $A^{8}$, as shown in Figure 59. 
Notice that it follows from the symmetry of the diagrammatic recoupling formulas of Figure 57 that the square of the recoupling matrix $F$ is equal to the identity. That is,

$$
\begin{gathered}
\left(\begin{array}{ll}
1 & 0 \\
0 & 1
\end{array}\right)= \\
F^{2}=\left(\begin{array}{cc}
1 / \Delta & \Delta / \Theta \\
\Theta / \Delta^{2} & T \Delta / \Theta^{2}
\end{array}\right)\left(\begin{array}{cc}
1 / \Delta & \Delta / \Theta \\
\Theta / \Delta^{2} & T \Delta / \Theta^{2}
\end{array}\right)= \\
\left(\begin{array}{cc}
1 / \Delta^{2}+1 / \Delta & 1 / \Theta+T \Delta^{2} / \Theta^{3} \\
\Theta / \Delta^{3}+T /(\Delta \Theta) & 1 / \Delta+\Delta^{2} T^{2} / \Theta^{4}
\end{array}\right) .
\end{gathered}
$$

Thus we need the relation

$$
1 / \Delta+1 / \Delta^{2}=1
$$

This is equivalent to saying that

$$
\Delta^{2}=1+\Delta
$$

a quadratic equation whose solutions are

$$
\Delta=(1 \pm \sqrt{5}) / 2
$$

Furthermore, we know that

$$
\Delta=\delta^{2}-1
$$

from Figure 54. Hence

$$
\Delta^{2}=\Delta+1=\delta^{2} .
$$

We shall now specialize to the case where

$$
\Delta=\delta=(1+\sqrt{5}) / 2,
$$

leaving the other cases for the exploration of the reader. We then take

$$
A=e^{3 \pi i / 5}
$$

so that

$$
\delta=-A^{2}-A^{-2}=-2 \cos (6 \pi / 5)=(1+\sqrt{5}) / 2 .
$$

Note that $\delta-1 / \delta=1$. Thus

$$
\Theta=(\delta-1 / \delta)^{2} \delta-\Delta / \delta=\delta-1 .
$$

and

$$
\begin{gathered}
T=(\delta-1 / \delta)^{2}\left(\delta^{2}-2\right)-2 \Theta / \delta=\left(\delta^{2}-2\right)-2(\delta-1) / \delta \\
=(\delta-1)(\delta-2) / \delta=3 \delta-5 .
\end{gathered}
$$

Note that

$$
T=-\Theta^{2} / \Delta^{2},
$$

from which it follows immediately that

$$
F^{2}=I \text {. }
$$

This proves that we can satisfy this model when $\Delta=\delta=(1+\sqrt{5}) / 2$. 
For this specialization we see that the matrix $F$ becomes

$F=\left(\begin{array}{cc}1 / \Delta & \Delta / \Theta \\ \Theta / \Delta^{2} & T \Delta / \Theta^{2}\end{array}\right)=\left(\begin{array}{cc}1 / \Delta & \Delta / \Theta \\ \Theta / \Delta^{2} & \left(-\Theta^{2} / \Delta^{2}\right) \Delta / \Theta^{2}\end{array}\right)=\left(\begin{array}{cc}1 / \Delta & \Delta / \Theta \\ \Theta / \Delta^{2} & -1 / \Delta\end{array}\right)$

This version of $F$ has square equal to the identity independent of the value of $\Theta$, so long as $\Delta^{2}=\Delta+1$.

The Final Adjustment. Our last version of $F$ suffers from a lack of symmetry. It is not a symmetric matrix, and hence not unitary. A final adjustment of the model gives this desired symmetry. Consider the result of replacing each trivalent vertex (with three 2-projector strands) by a multiple by a given quantity $\alpha$. Since the $\Theta$ has two vertices, it will be multiplied by $\alpha^{2}$. Similarly, the tetradhedron $T$ will be multiplied by $\alpha^{4}$. The $\Delta$ and the $\delta$ will be unchanged. Other properties of the model will remain unchanged. The new recoupling matrix, after such an adjustment is made, becomes

$$
\left(\begin{array}{cc}
1 / \Delta & \Delta / \alpha^{2} \Theta \\
\alpha^{2} \Theta / \Delta^{2} & -1 / \Delta
\end{array}\right)
$$

For symmetry we require

$$
\Delta /\left(\alpha^{2} \Theta\right)=\alpha^{2} \Theta / \Delta^{2}
$$

We take

$$
\alpha^{2}=\sqrt{\Delta^{3}} / \Theta
$$

With this choice of $\alpha$ we have

$$
\Delta /\left(\alpha^{2} \Theta\right)=\Delta \Theta /\left(\Theta \sqrt{\Delta^{3}}\right)=1 / \sqrt{\Delta} .
$$

Hence the new symmetric $F$ is given by the equation

$$
F=\left(\begin{array}{cc}
1 / \Delta & 1 / \sqrt{\Delta} \\
1 / \sqrt{\Delta} & -1 / \Delta
\end{array}\right)=\left(\begin{array}{cc}
\tau & \sqrt{\tau} \\
\sqrt{\tau} & -\tau
\end{array}\right)
$$

where $\Delta$ is the golden ratio and $\tau=1 / \Delta$. This gives the Fibonacci model. Using Figures 58 and 59, we have that the local braiding matrix for the model is given by the formula below with $A=e^{3 \pi i / 5}$.

$$
R=\left(\begin{array}{cc}
-A^{4} & 0 \\
0 & A^{8}
\end{array}\right)=\left(\begin{array}{cc}
e^{4 \pi i / 5} & 0 \\
0 & -e^{2 \pi i / 5}
\end{array}\right)
$$

The simplest example of a braid group representation arising from this theory is the representation of the three strand braid group generated by $S_{1}=$ $R$ and $S_{2}=F R F$ (Remember that $F=F^{T}=F^{-1}$.). The matrices $S_{1}$ and $S_{2}$ are both unitary, and they generate a dense subset of the unitary group $U(2)$, supplying the first part of the transformations needed for quantum computing. 


\section{Quantum Computation of Colored Jones Polynomials and the Witten-Reshetikhin- Turaev Invariant}

In this section we make some brief comments on the quantum computation of colored Jones polynomials. This material will be expanded in a subsequent publication.

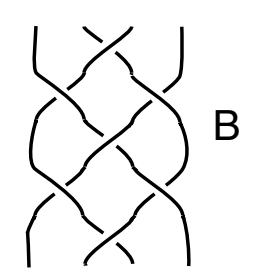

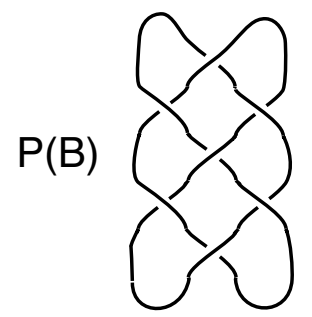

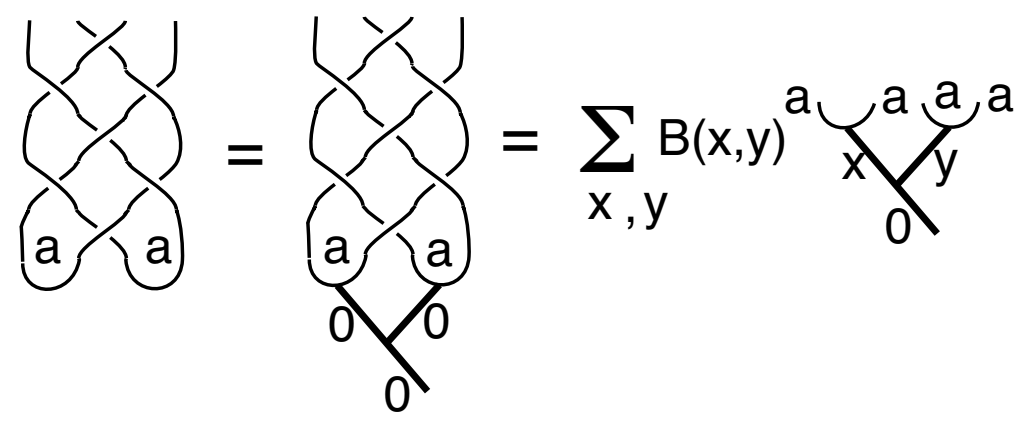

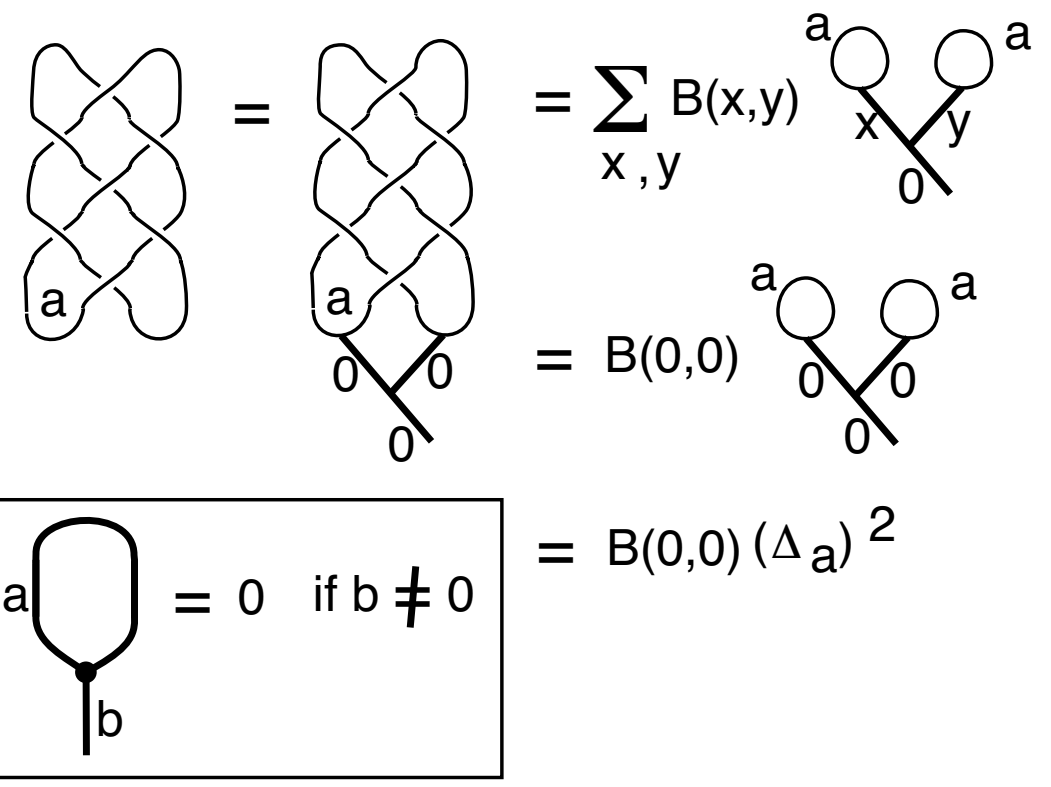

Figure 60 - Evaluation of the Plat Closure of a Braid 
First, consider Figure 60. In that figure we illustrate the calculation of the evalutation of the $(a)$ - colored bracket polynomial for the plat closure $P(B)$ of a braid $B$. The reader can infer the definition of the plat closure from Figure 60. One takes a braid on an even number of strands and closes the top strands with each other in a row of maxima. Similarly, the bottom strands are closed with a row of minima. It is not hard to see that any knot or link can be represented as the plat closure of some braid. Note that in this figure we indicate the action of the braid group on the process spaces corresponding to the small trees attached below the braids.

The $(a)$ - colored bracket polynonmial of a link $L$, denoted $\langle L\rangle_{a}$, is the evaluation of that link where each single strand has been replaced by a parallel strands and the insertion of Jones-Wenzl projector (as discussed in Section 7). We then see that we can use our discussion of the Temperley-Lieb recoupling theory as in sections 7,8 and 9 to compute the value of the colored bracket polynomial for the plat closure $P B$. As shown in Figure 60, we regard the braid as acting on a process space $V_{0}^{a, a, \cdots, a}$ and take the case of the action on the vector $v$ whose process space coordinates are all zero. Then the action of the braid takes the form

$$
B v(0, \cdots, 0)=\Sigma_{x_{1}, \cdots, x_{n}} B\left(x_{1}, \cdots, x_{n}\right) v\left(x_{1}, \cdots, x_{n}\right)
$$

where $B\left(x_{1}, \cdots, x_{n}\right)$ denotes the matrix entries for this recoupling transformation and $v\left(x_{1}, \cdots, x_{n}\right)$ runs over a basis for the space $V_{0}^{a, a, \cdots, a}$. Here $n$ is even and equal to the number of braid strands. In the figure we illustrate with $n=4$. Then, as the figure shows, when we close the top of the braid action to form $P B$, we cut the sum down to the evaluation of just one term. In the general case we will get

$$
<P B>_{a}=B(0, \cdots, 0) \Delta_{a}^{n / 2}
$$

The calculation simplifies to this degree because of the vanishing of loops in the recoupling graphs. The vanishing result is stated in Figure 60, and it is proved in the case $a=2$ in Figure 52.

The colored Jones polynomials are normalized versions of the colored bracket polymomials, differing just by a normalization factor.

In order to consider quantumn computation of the colored bracket or colored Jones polynomials, we therefore can consider quantum computation of the matrix entries $B(0, \cdots, 0)$. These matrix entries in the case of the roots of unity $A=e^{i \pi / 2 r}$ and for the $a=2$ Fibonacci model with $A=e^{3 i \pi / 5}$ are parts of the diagonal entries of the unitary transformation that represents the braid 
group on the process space $V_{0}^{a, a, \cdots, a}$. We can obtain these matrix entries by using the Hadamard test as described in section 4. As a result we get relatively efficient quantum algorithms for the colored Jones polynonmials at these roots of unity, in essentially the same framework as we described in section 4, but for braids of arbitrary size. The computational complexity of these models is essentially the same as the models for the Jones polynomial discussed in [1]. We reserve discussion of these issues to a subsequent publication.
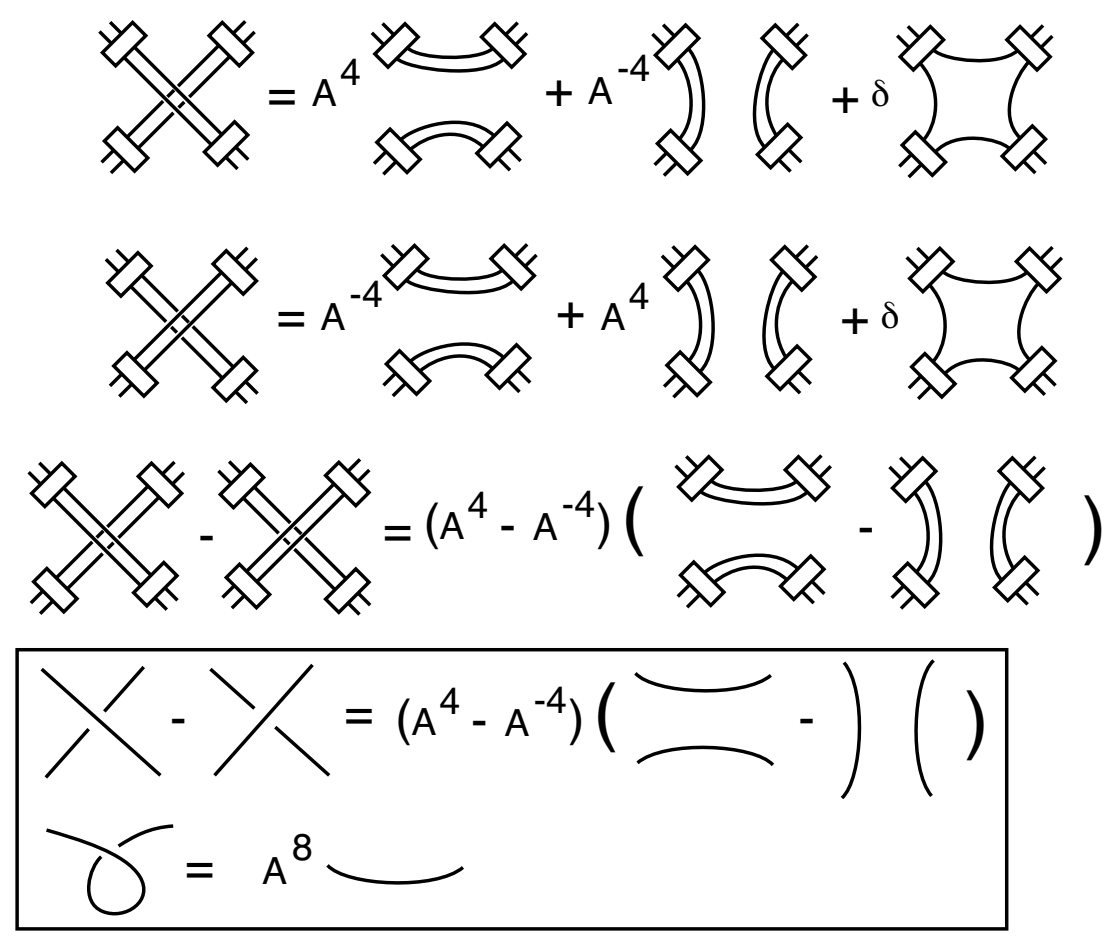

\section{Figure 61 - Dubrovnik Polynomial Specialization at Two Strands}

It is worth remarking here that these algorithms give not only quantum algorithms for computing the colored bracket and Jones polynomials, but also for computing the Witten-Reshetikhin-Turaev ( $W R T$ ) invariants at the above roots of unity. The reason for this is that the $W R T$ invariant, in unnormalized form is given as a finite sum of colored bracket polynomials:

$$
W R T(L)=\Sigma_{a=0}^{r-2} \Delta_{a}<L>_{a}
$$

and so the same computation as shown in Figure 60 applies to the $W R T$. This means that we have, in principle, a quantum algorithm for the computation 
of the Witten functional integral [87] via this knot-theoretic combinatorial topology. It would be very interesting to understand a more direct approach to such a computation via quantum field theory and functional integration.

Finally, we note that in the case of the Fibonacci model, the (2)-colored bracket polynomial is a special case of the Dubrovnik version of the Kauffman polynomial [41]. See Figure 61 for diagammatics that resolve this fact. The skein relation for the Dubrovnik polynomial is boxed in this figure. Above the box, we show how the double strands with projectors reproduce this relation. This observation means that in the Fibonacci model, the natural underlying knot polynomial is a special evaluation of the Dubrovnik polynomial, and the Fibonacci model can be used to perform quantum computation for the values of this invariant.

\section{References}

[1] D. Aharonov, V. Jones, Z. Landau, A polynomial quantum algorithm for approximating the Jones polynomial, quant-ph/0511096.

[2] D. Aharonov, I. Arad, The BQP-hardness of approximating the Jones polynomial, quant-ph/0605181.

[3] Y.Akutsu and M.Wadati. Knot invariants and critical statistical systems. J.Phys.Soc.Japan 56 (1987)839-842.

[4] J.W.Alexander. Topological invariants of knots and links.Trans.Amer.Math.Soc. 20 (1923) .275-306.

[5] P. K. Aravind, Borromean entanglement of the GHZ state. in 'Potentiality, Entanglement and Passion-at-a-Distance", ed. by R. S. Cohen et al, pp. 53-59, Kluwer, 1997.

[6] M.F. Atiyah, The Geometry and Physics of Knots, Cambridge University Press, 1990.

[7] Ashtekar,Abhay, Rovelli, Carlo and Smolin,Lee [1992], "Weaving a Classical Geometry with Quantum Threads", Phys. Rev. Lett., vol. 69, p. 237.

[8] A. Ashetekar and J. Lewandowski, Quantum theory of geometry I: Area operators, Class. Quant. Grav. 14 (1997), A55-A81.

[9] Baez,John and Muniain, Javier P. Gauge Fields, Knots and Gravity, World Scientific Series on Knots and Everything, Vol. 4 (1994). 
[10] R.J. Baxter. Exactly Solved Models in Statistical Mechanics. Acad. Press (1982).

[11] D. Bar-Natan, On the Vassiliev knot invariants, Topology 34 (1995), 423472.

[12] Dror Bar-Natan, Perturbative Aspects of the Chern-Simons Topological Quantum field Theory, Ph. D. Thesis, Princeton University, June 1991.

[13] G. Benkart, Commuting actions - a tale of two groups, in "Lie algebras and their representations (Seoul 1995)", Contemp. Math. Series, Vol. 194, American Mathematical Society (1996), pp. 1-46.

[14] J. Birman and X.S.Lin, Knot polynomials and Vassilievs invariants, Invent. Math. 111 No. 2 (1993), 225-270.

[15] N. E. Bonesteel, L. Hormozi, G. Zikos and S. H. Simon, Braid topologies for quantum computation, quant-ph/0505665.

[16] S. H. Simon, N. E. Bonesteel, M. H. Freedman, N. Petrovic and L. Hormozi, Topological quantum computing with only one mobile quasiparticle, quant-ph/0509175.

[17] J. L. Brylinski and R. Brylinski, Universal quantum gates, in Mathematics of Quantum Computation, Chapman \& Hall/CRC Press, Boca Raton, Florida, 2002 (edited by R. Brylinski and G. Chen).

[18] Chen, G., L. Kauffman, and S. Lomonaco, (eds.), "Mathematics in Quantum Computation and Quantum Technology," Chapman \& Hall/CRC , (2007).

[19] L. Crane, 2-d physics and 3-d topology, Comm. Math. Phys. 135 (1991), no. 3, 615-640.

[20] B. Coecke, The logic of entanglement, quant-phy/0402014.

[21] S. Abramsky and B. Coecke, A categorical semantics of quantum protocols, quant-ph/0402130.

[22] C. Dewitt-Morette, P. Cartier and A. Folacci, Functional Integration Basics and Applications, NATO ASI Series, Series B: Physics Vol. 361 (1997).

[23] P.A.M. Dirac, Principles of Quantum Mechanics, Oxford University Press, 1958. 
[24] V.G.Drinfeld. Quantum Groups, Proc.Intl.Congress Math.,Berkeley,Calif.USA(1986).789-820.

[25] H. Dye, Unitary solutions to the Yang-Baxter equation in dimension four. arXiv:quant-ph/0211050 v2 22 January 2003.

[26] E. Fradkin and P. Fendley, Realizing non-abelian statistics in timereversal invariant systems, Theory Seminar, Physics Department, UIUC, $4 / 25 / 2005$.

[27] M. Freedman, A magnetic model with a possible Chern-Simons phase, quant-ph/0110060v1 9 Oct 2001, (2001), preprint

[28] M. Freedman, Topological Views on Computational Complexity, Documenta Mathematica - Extra Volume ICM, 1998, pp. 453-464.

[29] M. Freedman, M. Larsen, and Z. Wang, A modular functor which is universal for quantum computation, quant-ph/0001108v2, 1 Feb 2000.

[30] M. H. Freedman, A. Kitaev, Z. Wang, Simulation of topological field theories by quantum computers, Commun. Math. Phys., 227, 587-603 (2002), quant-ph/0001071.

[31] M. Freedman, Quantum computation and the localization of modular functors, quant-ph/0003128.

[32] J. Fröhlich and C. King, The Chern Simons Theory and Knot Polynomials, Commun. Math. Phys. 126 (1989), 167-199.

[33] C. Frohman and J. Kania-Bartoszynska, $S O(3)$ topological quantum field theory, Comm. Anal. Geom. 4, (1996), no. 4, 589-679.

[34] V.F.R. Jones, A polynomial invariant for links via von Neumann algebras, Bull. Amer. Math. Soc. 129 (1985), 103-112.

[35] V.F.R.Jones. Hecke algebra representations of braid groups and link polynomials. Ann. of Math. 126 (1987), pp. 335-338.

[36] V.F.R.Jones. On knot invariants related to some statistical mechanics models. Pacific J. Math., vol. 137, no. 2 (1989), pp. 311-334.

[37] L.H. Kauffman, State models and the Jones polynomial, Topology 26 (1987), 395-407.

[38] L.H. Kauffman, Statistical mechanics and the Jones polynomial, AMS Contemp. Math. Series 78 (1989), 263-297. 
[39] L.H. Kauffman, Temperley-Lieb Recoupling Theory and Invariants of Three-Manifolds, Princeton University Press, Annals Studies 114 (1994).

[40] L.H.Kauffman, New invariants in the theory of knots, Amer. Math. Monthly, Vol.95,No.3,March 1988. pp 195-242.

[41] L. H. Kauffman, An invariant of regular isotopy, Trans. Amer. Math. Soc. 318 (1990), no. 2, 417-471.

[42] L.H.Kauffman and P.Vogel, Link polynomials and a graphical calculus, Journal of Knot Theory and Its Ramifications, Vol. 1, No. 1,March 1992, pp. 59- 104.

[43] L.H. Kauffman (ed.), The Interface of Knots and Physics, AMS PSAPM, Vol. 51, Providence, RI, 1996.

[44] L.H. Kauffman, Knots and Physics, World Scientific Publishers (1991), Second Edition (1993), Third Edition (2002).

[45] L.H.Kauffman and D.E.Radford. Invariants of 3-manifolds derived from finite dimensional Hopf algebras. Journal of Knot Theory and its Ramifications, Vol.4, No.1 (1995), pp. 131-162.

[46] L. H. Kauffman, Functional Integration and the theory of knots, J. Math. Physics, Vol. 36 (5), May 1995, pp. 2402 - 2429.

[47] L. H. Kauffman, Witten's Integral and the Kontsevich Integrals, in Particles, Fields, and Gravitation, Proceedings of the Lodz, Poland (April 1998) Conference on Mathematical Physics edited by Jakub Remblienski, AIP Conference Proceedings 453 (1998), pp. 368 -381.

[48] L. H. Kauffman Knot Theory and the heuristics of functional integration, Physica A 281 (2000), 173-200.

[49] L.H. Kauffman, Quantum computing and the Jones polynomial, math.QA/0105255, in Quantum Computation and Information, $\mathrm{S}$. Lomonaco, Jr. (ed.), AMS CONM/305, 2002, pp. 101-137.

[50] L.H. Kauffman and S. J. Lomonaco Jr., Quantum entanglement and topological entanglement, New Journal of Physics 4 (2002), 73.1-73.18 (http://www.njp.org/).

[51] L. H. Kauffman, Teleportation Topology, quant-ph/0407224, (in the Proceedings of the 2004 Byelorus Conference on Quantum Optics), Opt. Spectrosc. 9, 2005, 227-232. 
[52] L. H. Kauffman, math.GN/0410329, Knot diagrammatics. "Handbook of Knot Theory ", edited by Menasco and Thistlethwaite, 233-318, Elsevier B. V., Amsterdam, 2005.

[53] L. H. Kauffman and T. Liko, hep-th/0505069, Knot theory and a physical state of quantum gravity, Classical and Quantum Gravity, Vol 23, ppR63 (2006).

[54] L.H. Kauffman and S. J. Lomonaco Jr., Entanglement Criteria - Quantum and Topological, in Quantum Information and Computation - Spie Proceedings, 21-22 April, 2003, Orlando, FL, Donkor, Pinch and Brandt (eds.), Volume 5105, pp. 51-58.

[55] L. H. Kauffman and S. J. Lomonaco Jr., Quantum knots, in Quantum Information and Computation II, Proceedings of Spie, 12-14 April 2004 (2004), ed. by Donkor Pirich and Brandt, pp. 268-284.

[56] L. H. Kauffman and S. J. Lomonaco, Braiding Operators are Universal Quantum Gates, New Journal of Physics 6 (2004) 134, pp. 1-39.

[57] L. H. Kauffman and S. J. Lomonaco Jr., Spin Networks and Anyonic Topological Quantum Computing, quant-ph/0603131 v3 Apr 2006 (to appear in the Spie Proceedings 2006).

[58] L. H. Kauffman and S. J. Lomonaco Jr., $q$ - Deformed Spin Networks, Knot Polynomials and Anyonic Topological Quantum Computation, quant-ph/0606114.

[59] L. H. Kauffman and S. J. Lomonaco Jr., Topological quantum computing and the Jones polynomial, quant-ph/0605004.

[60] S. J. Lomonaco and L.H. Kauffman, Quantum Knots and Mosaics, Journal of Quantum Information Processing, vol. 7, Nos. 2-3, (2008), 85-115.

[61] L. H. Kauffman (editor), "Knots and Applications", (1996) World Scientific Pub. Co.

[62] A. Kitaev, Anyons in an exactly solved model and beyond, arXiv.condmat/0506438 v1 17 June 2005.

[63] H. Kleinert, Path Integrals in Quantum Mechanics, Statistics and Polymer Physics, 2nd edition, World Scientific, Singapore (1995).

[64] H. Kleinert, Grand Treatise on Functional Integration, World Scientific Pub. Co. (1999). 
[65] T. Kohno, Conformal Field Theory and Topology, AMS Translations of Mathematical Monographs, Vol 210 (1998).

[66] J. M. F. Labastida and E. Pérez, Kontsevich Integral for Vassiliev Invariants from Chern-Simons Perturbation Theory in the Light-Cone Gauge, J. Math. Phys., Vol. 39 (1998), pp. 5183-5198.

[67] A. Marzuoli and M. Rasetti, Spin network quantum simulator, Physics Letters A 306 (2002) 79-87.

[68] S. Garnerone, A. Marzuoli, M. Rasetti, Quantum automata, braid group and link polynomials, quant-ph/0601169

[69] S. A. Major, A spin network primer, arXiv:gr-qc/9905020.

[70] G. Moore and N. Seiberg, Classical and quantum conformal field theory, Comm. Math. Phys. 123 (1989), no. 2, 177-254.

[71] M. A. Nielsen and I. L. Chuang, "Quantum Computation and Quantum Information," Cambrige University Press, Cambridge (2000).

[72] R. Penrose, Angular momentum: An approach to Combinatorial Spacetime, In Quantum Theory and Beyond, edited by T. Bastin, Cambridge University Press (1969).

[73] J. Preskill, Topological computing for beginners, (slide presentation), Lecture Notes for Chapter 9 - Physics 219 - Quantum Computation. http://www.iqi.caltech.edu/preskill/ph219

[74] P. Cotta-Ramusino,E.Guadagnini,M.Martellini,M.Mintchev, Quantum field theory and link invariants, Nucl. Phys. B 330, Nos. 2-3 (1990), pp. $557-574$

[75] N.Y. Reshetikhin and V. Turaev. Ribbon graphs and their invariants derived from quantum groups. Comm. Math. Phys. 127 (1990). pp. 1-26.

[76] N.Y. Reshetikhin and V. Turaev. Invariants of Three Manifolds via link polynomials and quantum groups. Invent. Math. 103, 547-597 (1991).

[77] M. Roetteles, (private conversation, fall 2003).

[78] C. Rovelli and L. Smolin, Spin networks and quantum gravity, Phys Rev. D 52 (1995), 5743-5759.

[79] B. Schumacher, Ph.D. Thesis. 
[80] V. V. Shende, S. S. Bullock and I. L. Markov, Recognizing small circuit structure in two-qubit operators, (arXiv:quant-ph/030845 v2 8 Aug 2003)

[81] C. Ernst, D.W. Sumners, A calculus for rational tangles: Applications to DNA Recombination, Math. Proc. Camb. Phil. Soc., 108 (1990), 489-515.

[82] G. Spencer-Brown, "Laws of Form," George Allen and Unwin Ltd. London (1969).

[83] V.G.Turaev. The Yang-Baxter equations and invariants of links. LOMI preprint E-3-87, Steklov Institute, Leningrad, USSR. Inventiones Math. 92 Fasc.3,527-553.

[84] V.G. Turaev and O. Viro. State sum invariants of 3-manifolds and quantum 6j symbols. Topology, Vol. 31, No. 4, pp. 865-902 (1992).

[85] Lee Smolin, Link polynomials and critical points of the Chern-Simons path integrals, Mod. Phys. Lett. A, Vol. 4,No. 12, 1989, pp. 1091-1112.

[86] F. Wilczek, Fractional Statistics and Anyon Superconductivity, World Scientific Publishing Company (1990).

[87] E. Witten, Quantum field Theory and the Jones Polynomial, Commun. Math. Phys.,vol. 121, 1989, pp. 351-399.

[88] P. Wocjan, J. Yard The Jones polynomial: quantum algorithms and applications in quantum complexity theory, quant-ph/0603069.

[89] C. N. Yang, Phys. Rev. Lett. 19 (1967) 1312.

[90] Y. Zhang, L.H. Kauffman and M. L. Ge, Yang-Baxterizations, universal quantum gates and Hamiltonians. Quantum Inf. Process. 4 (2005), no. 3, $159-197$. 